\title{
Evolution Via EPR-Entanglement Algorithm
}

\author{
W. Grant Cooper \\ International Physics Health \& Energy, Inc. Lubbock, Texas, USA \\ And \\ Texas Tech University, College of Education, Lubbock, Texas \\ cooperwg@sbcglobal.net; willis.cooper@ttu.edu
}

\begin{abstract}
By quantifying EPR-generated accumulations of entangled proton qubits populating duplex microsatellite base pairs, observed as G-C $\rightarrow \mathbf{G}^{\prime}-\boldsymbol{C}^{\prime}, \mathrm{G}-\mathrm{C} \rightarrow{ }^{*} \boldsymbol{G}-\boldsymbol{C}^{*}$ and $\mathrm{A}-\mathrm{T} \rightarrow{ }^{*} \boldsymbol{A}-{ }^{*} \boldsymbol{T}$, the potential to exhibit expansion or contraction over evolutionary times can be qualitatively specified. Bold italics identify base pair superpositions of entangled proton qubits. Metastable hydrogen bonding amino $\left(-\mathrm{NH}_{2}\right)$ protons encounter quantum uncertainty limits, $\Delta x \Delta \mathrm{p}_{\mathrm{x}} \geq \hbar / 2$, which generate EPR arrangements, keto-amino -(entanglement) $\rightarrow$ enol-imine, yielding reduced energy entangled proton qubits shared between two indistinguishable sets of electron lone-pairs belonging to enol oxygen and imine nitrogen on opposite strands. When measured by Grover's-type quantum processors, $\delta \mathrm{t} \leq 10^{-13} \mathrm{~s}$, microsatellites whose entangled proton qubits generate a preponderance of initiation codons - UUG, CUG, AUG, GUG participate in the expansion mode of DNA synthesis, but if more stop codons - UAA, UGA, UAG - were introduced and/or the particular sequence consisted exclusively of A-T, such microsatellites would generally decrease in relative abundance over evolutionary times. This model is tested by evaluating the twenty-two most abundant microsatellites common to human and rat. From this list, predictions by "measurements of" entangled proton qubit states identify two ordered sets - eleven exhibiting expansion and eleven exhibiting contraction - of microsatellites, consistent with observation. These analyses imply Grover's-type enzyme-processor measurements of EPR-generated entangled proton "qubit pairs" can simulate microsatellite evolution, and further, identify entangled proton "qubit pairs" as the smallest "measurable" genetic informational unit, specifying particular evolution instructions with "measured" quantum information. Classical pathways cannot simulate microsatellite evolution observables.
\end{abstract}

Key Words: Entanglement evolution algorithm; Informational entangled qubits; Quantum/classical interface; Quantum information processing; Quantum uncertainty limits; Variable 'tick rate' clock

\section{Introduction}

Recent studies [1-4] of time-dependent molecular clock [5-11] genetic alterations are consistent with Grover's-type [12] enzyme quantum-readers measuring, $\delta \mathrm{t} \leq 10^{-13} \mathrm{~s}$, EPR-generated [13-18] entangled proton qubit states to yield time-dependent substitutions [6-9], $t s$, and time-dependent deletions [10-11], $\boldsymbol{t} \boldsymbol{d}$, after quantum information processing, $\Delta \mathrm{t}^{\prime} \leq 10^{-14} \mathrm{~s}$ [3], events of (i) transcription, (ii) translation, (iii) 
selection of accessible amino acids for peptide bond formation, (iv) initiation of genome growth and (v) random genetic drift [1-4,19]. Accuracy of this quantum entanglement algorithm model [1-4] for describing molecular evolution of DNA systems is tested by comparing its predictions to observation regarding evolutionary distributions of the twenty-two most abundant microsatellites common to rat and human genomes [20-22], identified in Table 1.

Microsatellites of length $L$ are short $(20 \leq L \leq 80 \mathrm{bp}$ ) tandem repeats (STRs) of duplex DNA with repeat unit $\leq 6$ bp [20-21]. Hundreds of thousands of STRs are distributed throughout eukaryote and prokaryote genomes [23-24] and have become a primary source of nuclear genetic markers for a variety of applications [25-27]. Because of considerable variability in repeat number at most loci, i.e., polymorphism, microsatellites are frequently used in the study of evolution and mapping of heritable disease genes [2834]. Studies on the origin, evolution and instability of such gene [34-37] have employed linkage disequilibrium analysis that is dependent on microsatellite mutation rates. Microsatellites generally exhibit mutation by the gain and/or loss of repeat units with an occasional point mutation interruption [38-40]. Although microsatellite evolution is of considerable interest [41-44], the responsible mechanisms are incompletely understood [45-48]. Specifically, the relative distribution of microsatellites - and their individual lengths - throughout eukaryote and prokaryote genomes have remained an enigma [20-23, 4950]. Also, microsatellite evolution rates may be different for humans, non-human primates and rodents, implying variable, species dependent mutation rates in STRs [40-41,51-54]. The effectiveness of resolving microsatellite data in evolutionary and genetic studies is a function of the accuracy of models for microsatellite evolution [1-2]. Inadequate models can reduce analytical insight and yield misleading conclusions [1-5]. The development of accurate models requires a proper understanding of mechanisms responsible for intra-loci and extra-loci dynamic events, and their consequences [1-3,28-29], which can cause expansions and/or contractions within microsatellite loci.

Models for microsatellite evolution have employed various combinations of slippage-like mutation $[38,48,55-56]$, rate of base substitution in flanking sequences [33,36], and the stepwise mutation model [57]. Nevertheless, the above mentioned models [24-28,30-48,55-57] for STR evolution have not provided an obvious rationale for the distribution of the 22 most abundant STRs common to human and rat genomes [20], displayed in Table 1. The purpose of this report is to predict, qualitatively, the evolutionary distribution of the 22 most abundant STRs (Table 1) common to rat and human DNA [20], using an evolutionarily selected, EPR-generated [13-18] quantum entanglement algorithm [1-4] for specifying time-dependent substitutions, ts, [5-9] and time-dependent deletions, td [10-11]. Quantum entanglement algorithm predictions [1-4] of STR expansion and/or contraction [6-7,29] herein considered generate favorable results, compatible with observation [20].

Recognition of entangled proton qubit resources [1-2] in ancestral ribozyme - RNA duplex segments [3-4] provides entanglement-enabled pathways for reactive proton qubits to implement incremental improvements to genome fitness, of the form: RNA - ribozyme $\rightarrow$ RNA - protein $\rightarrow$ DNA - protein [1-4]. This model implies enzyme-processor measurements of entangled proton qubit states, $\delta \mathrm{t} \leq 10^{-13} \mathrm{~s}$, can be responsible for initiating systematic origin of life evolutionary processes [2-4], and insight into manifestation of several age-related human diseases [1-2,6-8,29]. A subset of STRs includes unstable triplet repeats responsible for human genetic disease [1-2,6-8,28-29,33,46], where associated molecular 
W. Grant Cooper; Evolution Via EPR-Entanglement Algorithm. Journal of Biomedical Engineering and Medical Imaging, Volume 4, No 2, April (2017), pp 43-95

DNA dynamics can be qualitatively described in terms of EPR-generated [13-18] entangled proton qubits (Figs. 1-3) [1-4] - keto-amino $\rightarrow$ (entanglement) $\rightarrow$ enol-imine -

Table 1. Twenty-two most abundant microsatellites in rat and human genomes Microsatellite Sequences within Rat and Human Genome Databases*

\begin{tabular}{|c|c|c|c|c|c|c|}
\hline & 1 & 2 & 3 & 4 & 5 & 6 \\
\hline & $\begin{array}{l}\text { Rat } \\
\text { Motif }\end{array}$ & $\begin{array}{l}\text { No. } \\
\text { bp }>60\end{array}$ & $\begin{array}{c}\text { Total } \\
\text { Rat }\end{array}$ & $\begin{array}{l}\text { Human } \\
\text { Motif }\end{array}$ & $\begin{array}{c}\text { No. } \\
b p>40\end{array}$ & Total \\
\hline 1 & $\mathrm{CA}$ & 8 & 133 & A & 2 & 131 \\
\hline 2 & $\mathrm{CT}$ & 7 & 56 & $\mathrm{CA}$ & 10 & 73 \\
\hline 3 & A & 0 & 34 & А.Д.І & 3 & 21 \\
\hline 4 & A.AT & 0 & 18 & $\mathrm{CT}$ & 1 & 19 \\
\hline 5 & AAGG & 1 & 13 & $\mathrm{AT}$ & 3 & 16 \\
\hline 6 & $\mathrm{CAG}$ & 0 & 11 & $A, A G G$ & 7 & 15 \\
\hline 7 & AMAG & 1 & 8 & $A A C$ & 0 & 13 \\
\hline 8 & $\mathrm{AGAT}$ & 1 & 8 & $\triangle A, A C$ & 0 & 10 \\
\hline 9 & $A A C$ & 0 & 7 & $\mathrm{CCG}$ & 1 & 10 \\
\hline 10 & ACGC & 0 & 7 & $\mathrm{CAG}$ & 2 & 9 \\
\hline 11 & $\mathrm{AT}$ & 1 & 7 & $\mathrm{AGG}$ & 0 & 9 \\
\hline 12 & AGG & 1 & 6 & $\mathrm{AGAT}$ & 7 & 9 \\
\hline 13 & AATG & 0 & 6 & $\mathrm{~A} A \mathrm{GG}$ & 3 & 8 \\
\hline 14 & CAGA & 0 & 6 & ATCC & 3 & 7 \\
\hline 15 & $\mathrm{ACC}$ & 0 & 6 & AAT & 2 & 7 \\
\hline 16 & AAAC & 0 & 5 & A.ATC & 0 & 3 \\
\hline 17 & A.AT & 0 & 5 & AMTG & 0 & 3 \\
\hline 18 & AGGG & 0 & 5 & $\mathrm{ACAT}$ & 0 & 3 \\
\hline 19 & ACAT & 0 & 4 & $\mathrm{ACC}$ & 0 & 3 \\
\hline 20 & AATT & 0 & 3 & $\mathrm{CAGA}$ & 0 & 2 \\
\hline 21 & ATCC & 0 & 3 & $\mathrm{~A} A \mathrm{AG}$ & 1 & 2 \\
\hline 22 & CCG & 0 & 3 & ATC & 0 & 2 \\
\hline
\end{tabular}

*Adapted from Beckmann \& Weber[20]

which are measured and expressed by Grover's-type [1-2,12] quantum processors. Execution of quantum processor measurements, $\delta \mathrm{t} \leq 10^{-13} \mathrm{~s}$, of entangled proton qubit states yields time-dependent substitutions, ts [6-9], and time-dependent deletions, td [10-11], which can cause time-dependent introduction, and deletion, of initiation codons - UUG, CUG, AUG, GUG - and/or termination codons: UAG, UGA, UAA [1-2,6,29,58]. Execution of instructions specified by "recently introduced" codons can cause addition or subtraction of STRs for the particular microsatellite [1-2,6-7]. 

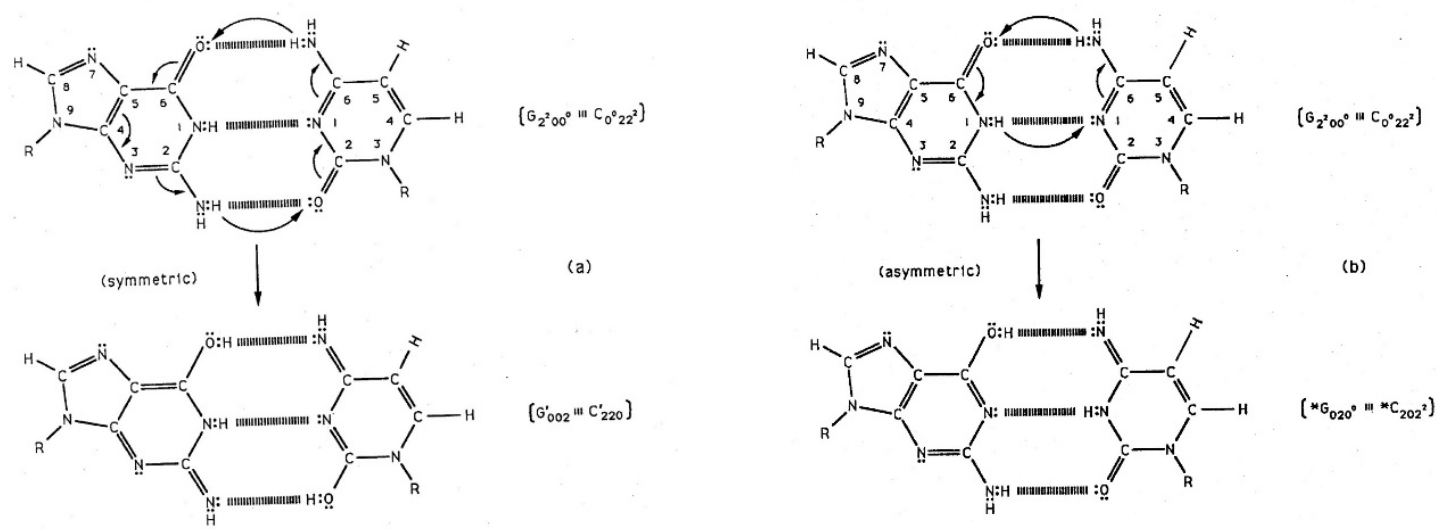

Figure 1. (a) Symmetric and (b) asymmetric channels for EPR proton exchange - electron arrangement at a GC site, creating entangled pairs of proton qubits.

(Figure 1. Symmetric (a) and asymmetric (b) channels for EPR proton exchange - electron arrangement at a G-C site. (a) Symmetric channel for proton exchange tunneling electron rearrangement, yielding two enol-imine hydrogen bonds between complementary $\mathbf{G}^{\prime}-\boldsymbol{C}^{\prime}$. Here an energetic guanine amino proton encounters quantum uncertainty limits, $\Delta x \Delta p_{x} \geq \hbar / 2$, and initiates the reaction. (b) The asymmetric exchange tunneling channel, yielding the ${ }^{*} \boldsymbol{G}-{ }^{*} \boldsymbol{C}$ "hybrid state" containing one enol-imine and one ketoamino hydrogen bond. An energetic cytosine amino proton initiates reaction in this channel. An annulus of reaction is identified by arrows within each G-C reactant duplex. Electron lone-pairs are represented by double dots, :. Bold italics, $\boldsymbol{G}^{\prime}-\boldsymbol{C}^{\prime}$ and ${ }^{*} \boldsymbol{G}-{ }^{*} \boldsymbol{C}$, denote necessity of Hilbert space to describe embedded entangled proton qubit dynamics.)

The initial half of this report - Sections I \& II - outlines the question regarding evolutionary distribution of STRs $[2,20]$ among rat and human, and presents an applicable quantum entanglement information processing model for "evolving" molecular DNA systems [1-4]. Model application includes illustrations that entanglement properties of proton qubit states are necessary for measurements by Grover's processors to accurately execute instructions specifying the particular $t s-G^{\prime} \mathbf{2} \mathbf{O} \mathbf{2} \rightarrow T, \mathbf{G}^{\prime} \mathbf{O} \mathbf{O} \mathbf{2} \rightarrow C^{*}{ }^{*} \mathbf{G O}$ $\mathbf{2} \mathbf{0}^{\boldsymbol{0}} \rightarrow \boldsymbol{A},{ }^{*} \boldsymbol{C} \mathbf{2} \mathbf{O} \mathbf{2}^{\mathbf{2}} \rightarrow T$ (see Table 2 \& Figs. 1-3 for notation) - or deletion, $\boldsymbol{t} \boldsymbol{d},{ }^{*} \boldsymbol{A} \rightarrow$ deletion \& ${ }^{*} \boldsymbol{T} \rightarrow$ deletion [1-2,6,11]. Quantum information processing analyses is developed to explain prokaryotic [3,911] systems, and is subsequently applied to eukaryotic data [1-2, 6-8,29]. In these cases, quantum uncertainty limits [59], $\Delta x \Delta p_{x} \geq \hbar / 2$, operate on metastable hydrogen bonded amino $\left(-\mathrm{NH}_{2}\right)$ protons, which introduces a probability of EPR-arrangements [13-18], keto-amino - (entanglement) $\rightarrow$ enol-imine (Figs. 1-3), observable as G-C $\rightarrow \boldsymbol{G}^{\prime}-\boldsymbol{C}^{\prime}, \mathrm{G}-\mathrm{C} \rightarrow{ }^{*} \boldsymbol{G}-{ }^{*} \boldsymbol{C}$ and A-T $\rightarrow{ }^{*} \boldsymbol{A}-{ }^{*} \boldsymbol{T}$ [1-4,6-11]. Product enol and imine protons - occupying $\boldsymbol{G}^{\prime}-\boldsymbol{C}^{\prime},{ }^{*} \boldsymbol{G}-{ }^{*} \boldsymbol{C},{ }^{*} \boldsymbol{A}-{ }^{*} \boldsymbol{T}$ - are entangled qubits [60-62] shared between two indistinguishable sets of electron lone-pairs belonging to enol oxygen and imine nitrogen on opposite genome strands (Fig. 2), and consequently, participate in entangled quantum oscillations $-|+>\rightleftarrows|->-$ at $\sim 10^{13} \mathrm{~s}^{-1}$ between near symmetric energy wells [1-2,6], in decoherence-free subspaces [63-65], until "measured by" evolutionarily selected Grover's-type [12] processors that implement quantum information processing. Bold italics $-\boldsymbol{G}^{\prime}-\boldsymbol{C}^{\prime},{ }^{*} \boldsymbol{G}-{ }^{*} \boldsymbol{C},{ }^{*} \boldsymbol{A}-{ }^{*} \boldsymbol{T}$ - are used to designate quantum enhanced genetic information embodied within dynamic entangled proton qubits occupying EPR-created [13-18] base pair sites. Section III employs quantum entanglement algorithm [1-3,6,29] applications for describing 
W. Grant Cooper; Evolution Via EPR-Entanglement Algorithm. Journal of Biomedical Engineering and Medical Imaging, Volume 4, No 2, April (2017), pp 43-95

molecular dynamics exhibited by "evolving" STRs $[1,6-7,20,29]$ listed in Table 1. Discussion and Conclusion of results obtained in Sec. III are given in Sec. IV.

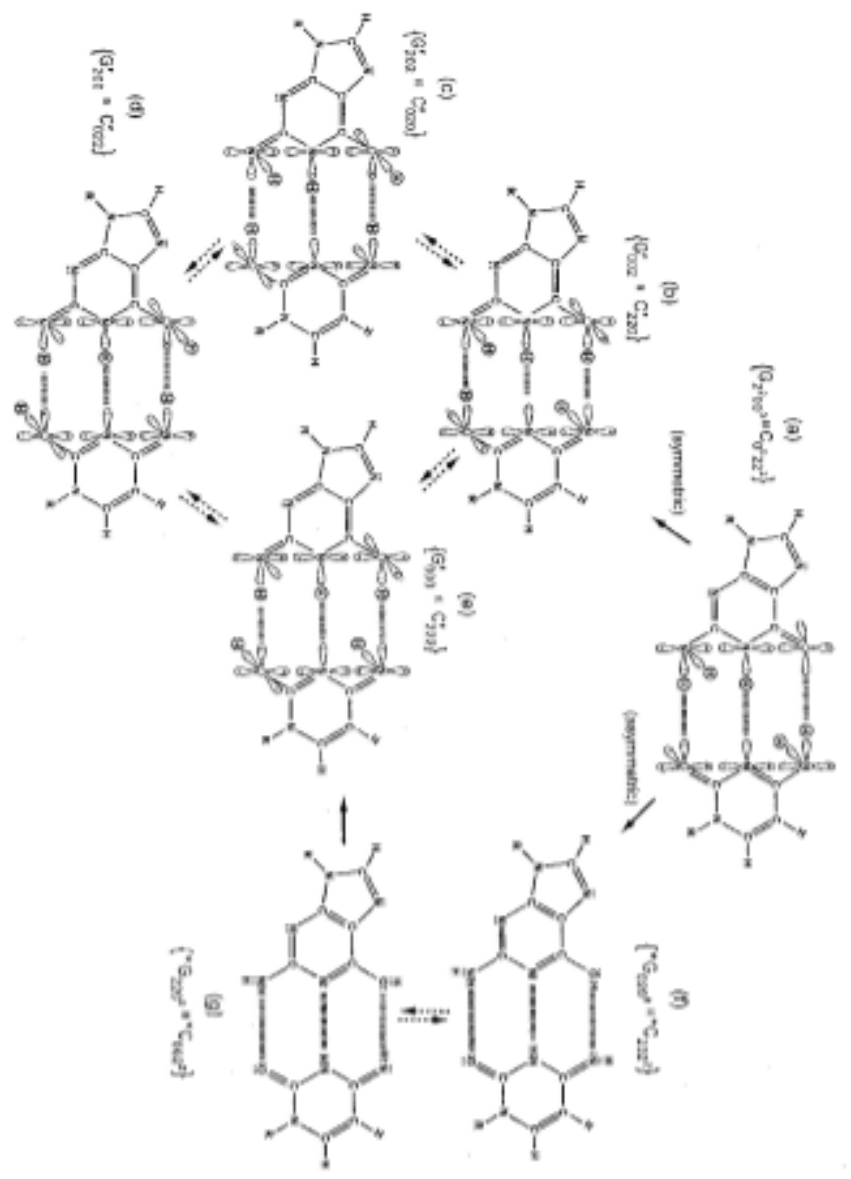

Figure 2. Distribution of observable entangled proton qubit states at a $G^{\prime}-C^{\prime}$ (symmetric) or ${ }^{*} G-{ }^{*} C$ (asymmetric) superposition site.

(Figure 2. Distribution of entangled proton qubit states at a $\mathbf{G}^{\prime}-\boldsymbol{C}^{\prime}$ (symmetric) or ${ }^{*} \mathbf{G}-{ }^{*} \boldsymbol{C}$ (asymmetric) superposition site. Symmetric, asymmetric and second asymmetric (unlabeled) channels $(\rightarrow)$ by which metastable keto-amino G-C protons populate enol and imine entangled proton qubit states. Dashed arrows identify pathways for quantum oscillation of enol and imine proton qubits. Approximate electronic structures for hydrogen bond end groups and corresponding proton positions are shown for the metastable keto-amino duplex (a) and for enol and imine entangled proton qubit states, $\boldsymbol{G}^{\prime}-\boldsymbol{C}^{\prime}$ (b-e). Electron lone-pairs are represented by double dots, :, and a proton by a circled H. Proton states are specified by a compact notation, using letters G, C, A, T for DNA bases with 2's and 0's identifying electron lone-pairs and protons, respectively, donated to the hydrogen bond by - from left to right - the 6-carbon side chain, the ring nitrogen and the 2-carbon side chain. Superscripts identify the component at the outside position (in major and minor groves) as either an amino group proton, designated by $0^{0}$, or a keto group electron lone-pair, indicated by $2^{2}$. Superscripts are suppressed for enol and imine groups.) 


\section{Molecular Genomic Evolution via EPR-Generated Proton Qubits Measured by Grover's Processors}

\subsection{Model Summary}

Icy comets colliding with a cooling pre-biotic Earth [66] $-\sim 4.3$ to 3.9 billion y ago - created impact synthetic processes [2-4] that ultimately generated "ribozyme-like" RNA polymers which could inefficiently duplicate a few molecular units of RNA [67-70]. Random classical processes [71] introduced energetically preferable hydrogen bonded base pairs [72] - including keto - amino hydrogen bonds between complementary RNA duplex segments. Consequently, quantum uncertainty limits, $\Delta x \Delta p_{x} \geq \hbar / 2$ $[6,59]$, operated on metastable amino $\left(-\mathrm{NH}_{2}\right)$ hydrogen bonded RNA protons, which introduced a probability of EPR-arrangement [1-3,13-18], keto-amino - (entanglement) $\rightarrow$ enol-imine (Figs. 1-3 for DNA), where reduced energy enol and imine product protons are each shared between two indistinguishable sets of intramolecular electron lone-pairs belonging to enol oxygen and imine nitrogen on opposite genome strands $[2,10]$, and thus, participate in entangled quantum oscillations at $\sim 4 \times 10^{13}$ $\mathrm{s}^{-1}\left(\sim 4800 \mathrm{~m} \mathrm{~s}^{-1}\right)$ [2,10], in decoherence-free subspaces [63-65], until "measured" in a genome groove [7375], $\delta \mathrm{t} \ll 10^{-13} \mathrm{~s}$, by an evolutionarily selected, "truncated" Grover's [3,12] quantum bio-processor. Before proton decoherence, $\tau_{D}<10^{-13} \mathrm{~s}$ [75-76], proton - processor entanglement states implement quantum information processing, $\Delta \mathrm{t}^{\prime} \leq 10^{-14} \mathrm{~s}$, including (i) transcription, (ii) translation, (iii) selection of accessible amino acids for peptide bond formation, (iv) initiation of genome growth and $(v)$ random genetic drift $[1-3,19]$. This specified peptide bond formation $-\sim 8$ to $16 \mathrm{KJ} / \mathrm{mole}[1-3,58]$ from proton decoherence - and the final, decohered molecular clock state, which is an observable time-dependent

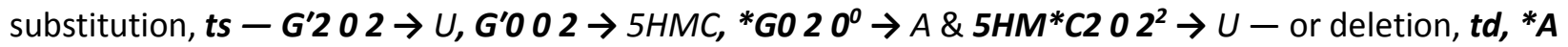
$\rightarrow$ deletion $\& * U \rightarrow$ deletion [1-3,9-11]. (See Table 2 for notation; here, primordial "RNA-type" genomes are assumed to have been composed of analogs of guanine, 5-hydroxymethylcytosine (5HMC), adenine and uracil [2-3].) RNA genomes containing "excessive" levels of entangled proton qubits were excluded from the viable gene pool [2-3], thereby allowing selection of an approximately "wild-type" gene pool. An evolved version of these ancestral "RNA-type" genes are identified as "gatekeeper" genes in Homo sapiens [1-4]. "Grover's-type" [12] quantum probability measurements of the 20 different available entangled proton qubit states [1-4] imply quantum entanglement origins of the triplet code, utilizing $4^{3}$ codons and $\sim 22$ L-amino acids. 

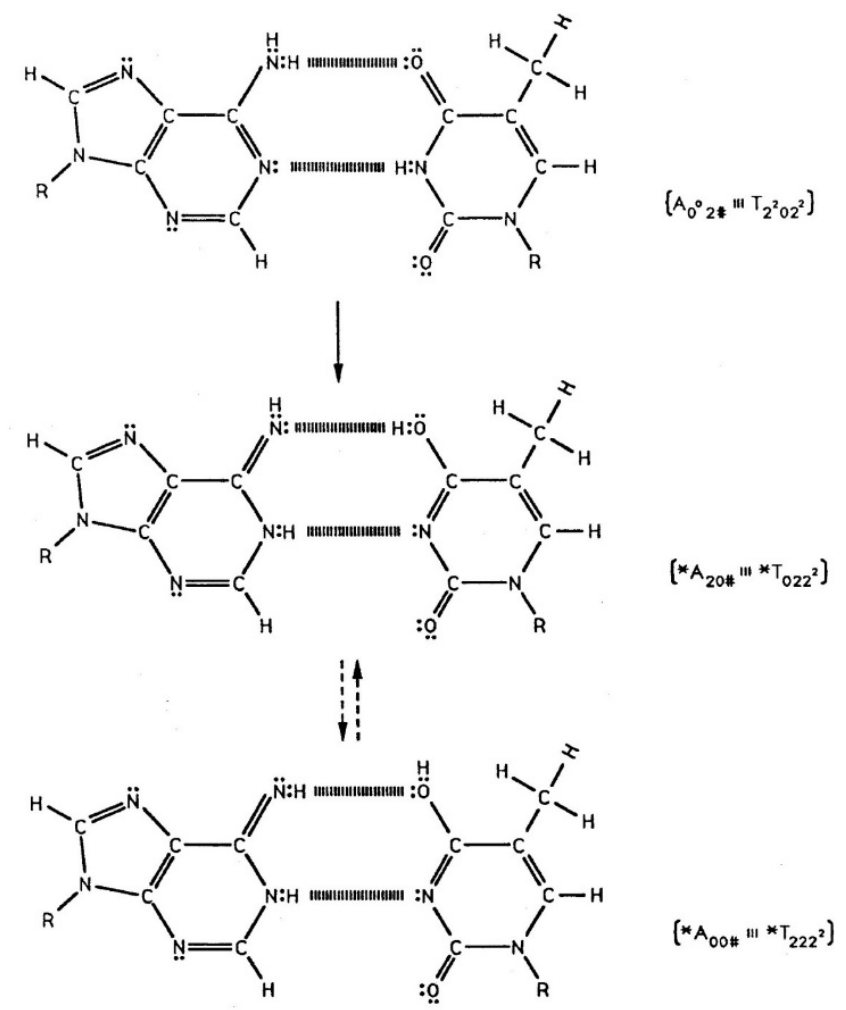

Fia.3WGromor

Figure 3. Metastable and proton qubit *A-*T states.

(Figure 3. Pathway for metastable keto-amino A-T protons to populate reduced energy enol and imine proton qubit states. Dashed arrows indicate proton oscillatory pathway for enol and imine proton qubit *A-*T states. Notation is given in Fig. 2 legend. The \# symbol indicates the position is occupied by ordinary hydrogen unsuitable for hydrogen bonding.)

When ancestral duplex RNA became too unwieldy for "error-free" duplication [2-3,67-70], newly selected repair enzymes replaced RNA with more suitable DNA [2-3,77]. Soon after genome conversion, RNA $\rightarrow$ DNA, uracil was replaced by 5-methyluracil (thymine) and $5 \mathrm{HMC}$ was generally replaced by cytosine. The quantum entanglement algorithm "measures", $\delta \mathrm{t} \leq 10^{-13} \mathrm{~s}$, entangled proton qubit states to yield $t$ and $\boldsymbol{t} \boldsymbol{d}$ into RNA and DNA genomes, after (i) an initial formation of enzyme-proton entanglement [1-3,12], $\delta \mathrm{t}$ $<10^{-13} \mathrm{~s}$, (ii) implementation of an entanglement-assisted enzyme quantum search $\left(\Delta \mathrm{t}^{\prime} \leq 10^{-14} \mathrm{~s}\right)$, (iii) specification of the "correct" complementary mispair [2,6,10-11], and (iv) selected replicationsubstitution or deletion [1-3], with classical tautomers containing decohered protons. Consistent with implementing steps - (i), (ii), (iii), (iv) - ts and $\boldsymbol{t} \boldsymbol{d}$ can introduce and eliminate initiation codons - UUG, CUG, AUG, GUG - and termination codons, UGA, UAA, UAG [1-2,6-7,29]. The resulting "dynamic mutations" $[1,29,78]$ can cause susceptible STRs, e.g., $(C A G)_{n}(n>36)$, to exhibit deletions and/or expansions $\geq 10$ (CAG) repeats in 20 y [1-2,33]. This observable expansion/contraction mechanism [1-2,6$8,29,33,78$ ] can account for genomic growth, over the past $\sim 3.5$ billion $y$, from primordial RNA [68] to $21^{\text {st }}$ century DNA of $\sim 6.8 \times 10^{9}$ base pairs $[1-3,58]$. 


\subsection{Molecular Genetic Observables Requiring Quantum Entanglement Algorithm Operations}

Recent studies of time-altered states exhibited by ancient [79] (a) T4 phage DNA [9-11,80-83], (b) human gene systems [1-2,4-8] and (c) human-rodent microsatellites [2,20] require enzymatic quantum information processing of entangled proton qubit states $[3,12]$ to satisfy observations. Convergence of these different studies imply evolutionary origins of quantum entanglement processing emerged during the era of ancestral ribozyme - RNA systems [2-3,67-70]. In these cases, the anti-entanglement hypothesis [84-85] disallowing required, ambient temperature in vivo entanglement states is falsified [1-4,6-11,8691]. Since lower energy enol and imine proton

Table 2

\begin{tabular}{|c|c|c|c|c|c|c|c|}
\hline \multirow{3}{*}{$\begin{array}{l}\text { Qubit } \\
\text { States }\end{array}$} & \multicolumn{6}{|c|}{ Allowable Pair Formation at Replication } & \multirow{3}{*}{$\begin{array}{c}\text { Transcription } \\
\text { Message }\end{array}$} \\
\hline & \multicolumn{4}{|c|}{ NORMAL BASES } & \multicolumn{2}{|c|}{ Syn-Purines } & \\
\hline & $\mathrm{G} 2^{2} 00^{\circ}$ & $\mathrm{C} 0^{0} 22^{2}$ & $\mathrm{~A} 0^{0} 2 \#$ & $\mathrm{~T} 2^{2} 02^{2}$ & $\mathrm{G}^{2} 2$ 2\# & $\mathrm{A} 0^{0} 2 \#$ & \\
\hline$G^{\prime} 002$ & & & & & $\mathrm{GC} \rightarrow \mathrm{CG}$ & & $\overline{\mathrm{U}^{+}}$ \\
\hline$G^{\prime} 202$ & & & & & & $\mathrm{GC} \rightarrow>\mathrm{TA}$ & $T 2^{2} 02^{2}$ \\
\hline$G^{\prime} 200$ & & $\begin{array}{c}\text { not } \\
\text { detectable }\end{array}$ & & & & & $G 2^{2} 00^{0}$ \\
\hline$G^{\prime} 000$ & & & & & & & $\mathrm{U}$ \\
\hline${ }^{*} \mathrm{G} 020^{\circ}$ & & & & $\mathrm{GC} \rightarrow \mathrm{AT}$ & & & $\overline{\mathrm{U}}$ \\
\hline${ }^{*} \mathrm{G} 220^{\circ}$ & & & & & & & $\mathrm{U}$ \\
\hline$C^{\prime} 220$ & & & & & & & $\mathrm{U}$ \\
\hline$C^{\prime} 020$ & & & & & & & $\mathrm{U}$ \\
\hline$C^{\prime} 022$ & $\begin{array}{c}\text { not } \\
\text { detectable }\end{array}$ & & & & & & $\mathrm{CO}^{2} 22^{2}$ \\
\hline$C^{\prime} 222$ & & & & & & & $\mathrm{U}$ \\
\hline${ }^{*} \mathrm{C} 202^{2}$ & & & $\mathrm{G}-\mathrm{C}-\mathrm{A} \mathrm{T}$ & & & & $T 2^{2} 02^{2}$ \\
\hline${ }^{*} \mathrm{C} 002^{2}$ & & & & & & & $\mathrm{U}$ \\
\hline *A20\# & & $\mathrm{A}-\mathrm{T} \rightarrow \mathrm{G}-\mathrm{C}$ & & & & $A-T->T-A$ & $\overline{\mathrm{U}}$ \\
\hline *A00\# & & & & & A-T $>$ C -G & & $\mathrm{U}$ \\
\hline${ }^{*} \mathrm{~T} 022^{2}$ & A-T_G-C & & & & & & $\mathrm{CO}^{2} 22^{2}$ \\
\hline${ }^{*} \mathrm{~T} 222^{2}$ & & & & & & & $\mathrm{U}$ \\
\hline
\end{tabular}

+Undefined 
W. Grant Cooper; Evolution Via EPR-Entanglement Algorithm. Journal of Biomedical Engineering and Medical Imaging, Volume 4, No 2, April (2017), pp 43-95

(Table 2. Transcribed messages from entangled proton qubit states, decohered isomers and formation of complementary, Topal-Fresco [9-11,92] mispairs. Normal tautomers (top row) and entangled qubit "flipflop" states/decohered tautomers (left column) are listed in terms of the compact notation for hydrogenbonding configurations identified in Fig. 2 Legend. Consistent with enzymatic quantum information processing, base pair substitution notation at the respective row-column juncture identifies eigenstate components that will form a complementary mispair with an incoming classical tautomer, selected by an enzyme-entanglement quantum search, $\Delta \mathrm{t}^{\prime} \leq 10^{-14} \mathrm{~s}$. Transcribed messages obtained from entangled proton qubit states are identified in the right hand column.)

qubit states [1-4,6-11] in metabolically inert, but biologically operational duplex genomes (RNA and DNA) [72] are initially unoccupied, but are energetically accessible [6-11], quantum confinement [59,93] of metastable hydrogen bonded amino $\left(-\mathrm{NH}_{2}\right)$ protons introduces EPR arrangement probabilities [13-18], keto-amino $\rightarrow$ enol-imine, observable as G-C $\rightarrow \boldsymbol{G}^{\prime}-\boldsymbol{C}^{\prime}, \mathrm{G}-\mathrm{C} \rightarrow{ }^{*} \boldsymbol{G}-{ }^{*} \boldsymbol{C}, \mathrm{A}-\mathrm{T} \rightarrow{ }^{*} \boldsymbol{A}-{ }^{*} \boldsymbol{T}$ (Figs. 1-3; Table 2), by transcription and replication of time-altered DNA lesions of T4 phage [6,9-11]. Transcription and replication of entangled proton qubit superposition $\boldsymbol{G}^{\prime}-\boldsymbol{C}^{\prime}$ and ${ }^{*} \boldsymbol{G}-{ }^{*} \boldsymbol{C}$ sites yield observable timedependent molecular clock base substitutions, $t s-G^{\prime} \mathbf{2} O \mathbf{2} \rightarrow T, \mathbf{G}^{\prime} \mathbf{O} \mathbf{O} \mathbf{2} \rightarrow C^{*}{ }^{*} \mathbf{G O} \mathbf{2} \mathbf{O}^{\circ} \rightarrow A \&{ }^{*} \mathbf{C} \mathbf{2} \mathbf{O} \mathbf{2}^{\mathbf{2}} \rightarrow$ $T[2,10]$ - whereas entangled proton qubit states within ${ }^{*} A-{ }^{*} T$ sites (Fig. 3 ) exhibit time-dependent deletions, $\boldsymbol{t} \boldsymbol{d},{ }^{*} \boldsymbol{A} \rightarrow$ deletion and ${ }^{*} \boldsymbol{T} \rightarrow$ deletion [11]. Also when $\boldsymbol{G}^{\prime}$ and/or ${ }^{*} \boldsymbol{C}$ is located on the transcribed strand, time-dependent substitutions, ts $-G^{\prime} \mathbf{2} \mathbf{O} \mathbf{2} \rightarrow T$ and ${ }^{*} \mathbf{C} \mathbf{2} \mathbf{O} \mathbf{2}^{\mathbf{2}} \rightarrow T$ - are expressed by quantum transcription before replication is initiated (Fig. 4) [10-11]. Subsequent replication - after entangled enzyme quantum searches, $\Delta \mathrm{t}^{\prime} \leq 10^{-14} \mathrm{~s}$ - expresses genotypically incorporated $\boldsymbol{t s}-\mathbf{G}^{\prime} \mathbf{2} \mathbf{0} \mathbf{2} \rightarrow T$ and ${ }^{*} \mathbf{C} \mathbf{2}$ $\mathbf{0} \mathbf{2}^{\mathbf{2}} \rightarrow T$ - at frequencies identical to those previously exhibited by quantum transcription before replication [10-11,81-82]. Therefore, $\boldsymbol{G}^{\prime} \rightarrow T$ and ${ }^{*} \boldsymbol{C} \rightarrow T$ contributions to the "gene pool" are 2 -fold $>$ "replication only" expectations, and further, replication incorporation ts instructions are provided by prior "Grover's-type" [2-3,12] transcriptase quantum measurements of entangled proton qubits. These "nonconventional", $\boldsymbol{G}^{\prime} \rightarrow T$ and ${ }^{*} \boldsymbol{C} \rightarrow T$ transcription and replication observations [10-11,81-82] are also required to explain evolutionary distributions of the 22 most abundant microsatellites (short tandem repeats, STRs) common to rat and human genomes $[2,20]$.

Classical restrictions [71] do not allow time-dependent mutations at G-C sites [2,6,9-11] to spontaneously accumulate in metabolically inert [94], extracellular T4 phage DNA [83] as point, heteroduplex heterozygotes, $r^{+} / r l l$ [80-82], G-C $\rightarrow \boldsymbol{G}^{\prime}-\boldsymbol{C}^{\prime}$ and $\mathrm{G}-\mathrm{C} \rightarrow{ }^{*} \boldsymbol{G}-{ }^{*} \boldsymbol{C}$ lesions, that subsequently express distinguishable $\boldsymbol{t s}$ observables, $\boldsymbol{G}^{\prime} \rightarrow T$ and ${ }^{*} \boldsymbol{C} \rightarrow T$, via transcription (and thus translation) before replication is initiated, and further, express the identical transcription-generated mutation frequencies $\boldsymbol{G}^{\prime} \rightarrow T$ and ${ }^{*} \boldsymbol{C} \rightarrow T$ - by subsequent replication-incorporated substitutions [10-11,81-82]. As noted elsewhere [2,9-11], these original observations [81-82] were viewed as enigmas [95-96], inconsistent with classical molecular genetics, and were ultimately ignored without resolution via classical molecular genetics [97]. Nevertheless, when $\boldsymbol{G}^{\prime}$ and/or ${ }^{*} \boldsymbol{C}$ are located on the transcribed strand, T4 phage $\boldsymbol{t s}$ systems [81-82] routinely exhibit identical $\boldsymbol{G}^{\prime} \rightarrow T$ and ${ }^{*} \boldsymbol{C} \rightarrow T$ mutation frequencies for pre-replication transcription, and post-transcription replication [10-11,97], implying non-classical pre-replication transcriptase processing of quantum informational content - occupying heteroduplex heterozygote $\mathbf{G}^{\prime}-\boldsymbol{C}^{\prime}$ and ${ }^{*} \boldsymbol{G}-{ }^{*} \boldsymbol{C}$ sites [80] - specifies frequencies of subsequent replication-implemented physical substitution mutations, $t s, \boldsymbol{G}^{\prime} \rightarrow T$ and ${ }^{*} \boldsymbol{C} \rightarrow T$ [2,10-11]. Also when the wild-type $r^{+}$allele requires a substitution, e.g., $\mathrm{G} \rightarrow \mathrm{T}$ or $\mathrm{C} \rightarrow \mathrm{T}$, for growth on $E$. coli $\mathrm{K}[80,98]$, quantum transcription of entangled proton qubits can 
generate quantum informational content, $\boldsymbol{G}^{\prime} \rightarrow T$ and/or ${ }^{*} \boldsymbol{C} \rightarrow T$, providing relevant "translated" information that specifies existence of the wild-type $r^{+}$allele, thereby allowing initiation of replication and subsequent growth [10-11]. In these cases, wild-type $r^{+}$allele requirements [80,98] are satisfied by translation of informational content generated by quantum transcription of EPR-generated entangled proton qubits, $\delta \mathrm{t}<<10^{-13} \mathrm{~s}$, not from physical molecular replacements, $\boldsymbol{G}^{\prime} \rightarrow T$ or ${ }^{*} \boldsymbol{C} \rightarrow T$, that occur in the ensuing round of replication [10-11]. In these situations, the observed translated messages from quantum transcriptions, e.g., G'202 $\rightarrow T$ and ${ }^{*} C \mathbf{2} 0 \mathbf{2}^{\mathbf{2}} \rightarrow T$ (Fig. 4), allow initiation of genome duplication, and thus, completes a feedback loop between an entangled enzyme-processor "measurement" of entangled proton qubit states, and subsequent genome growth [1-4,6-7,10-11].
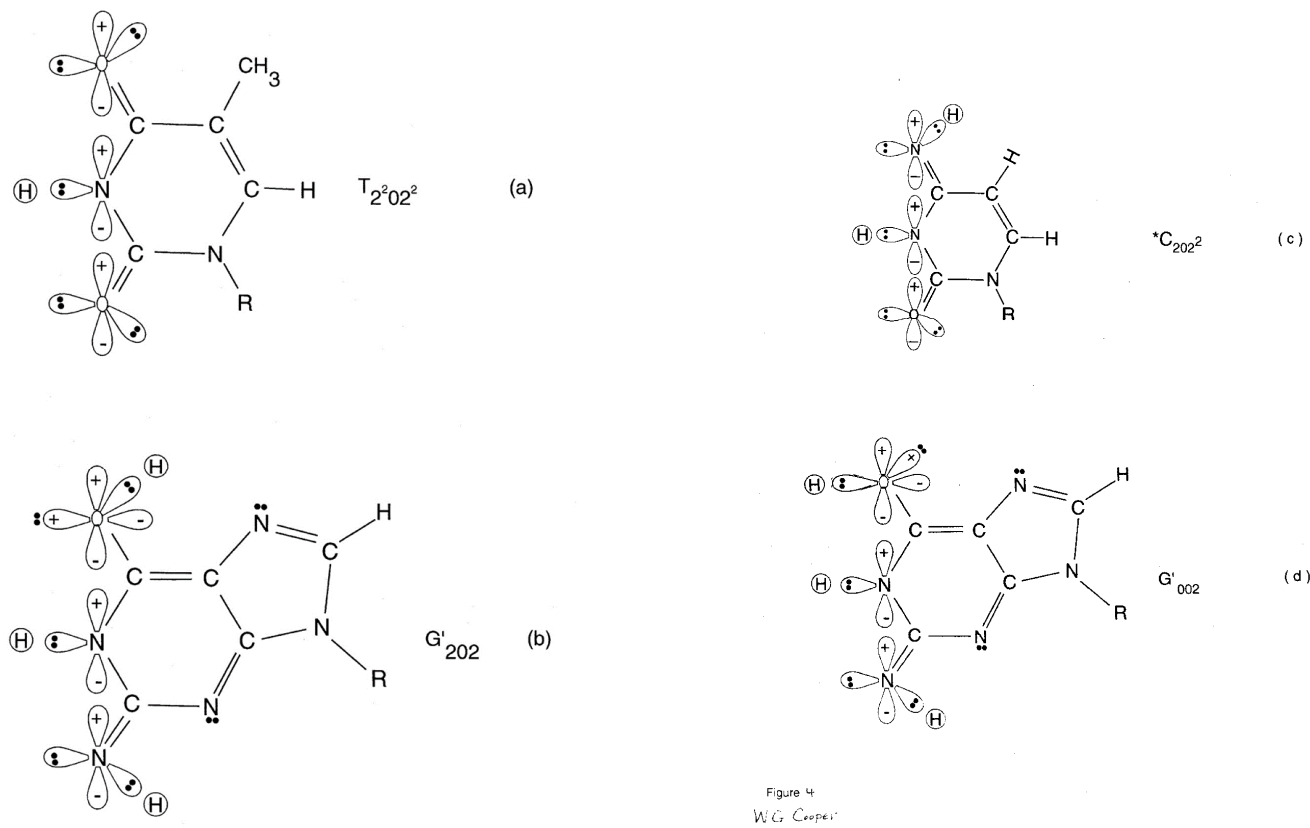

(b)

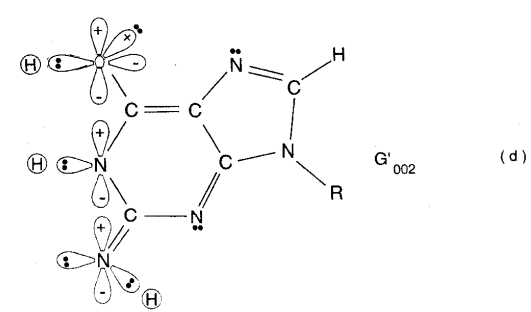

Figure 4
$W G$ Cooper

(Figure 4. Approximate proton-electron hydrogen bonding structure "seen by" Grover's [12] enzyme quantum reader in intervals, $\delta \mathrm{t} \ll<10^{-13} \mathrm{~s}$ )

Figure 4. Approximate proton-electron hydrogen bonding structure "seen by" Grover's [12] enzyme quantum reader in intervals, $\delta \mathrm{t} \ll 10^{-13} \mathrm{~s}$, encountering (a) normal thymine, $\mathrm{T}^{2} 02^{2}$; (b) enzymeentangled enol-imine $\boldsymbol{G}^{\prime} \mathbf{2} \mathbf{O}$ 2; (c) enzyme-entangled imino cytosine, ${ }^{*} \boldsymbol{C} \mathbf{2} \mathbf{O} \mathbf{2}^{\mathbf{2}}$, and (d) enzyme-entangled enol-imine $\mathbf{G}^{\prime} \mathbf{0} \mathbf{0} 2$. Notation is specified in Fig. 2 legend.

Enzyme entanglement conditions imposed on "measured", $\delta \mathrm{t}<<10^{-13} \mathrm{~s}$, coherent groove proton qubits of $\mathbf{G}^{\prime} \mathbf{2} \mathbf{O} \mathbf{2}$ and ${ }^{*} \mathbf{C} \mathbf{2} \mathbf{0} \mathbf{2}^{\mathbf{2}}$ create identical hydrogen bonding proton - electron lone-pair configurations for entangled $G^{\prime} \mathbf{2} 02$ and entangled *C2 $0 \mathbf{2}^{\mathbf{2}}$ (Fig. 4), as "viewed by" entangled transcriptase systems. Enzyme-entangled groove protons are dedicated to implementing the entanglement-assisted quantum search, $\Delta \mathrm{t}^{\prime} \leq 10^{-14} \mathrm{~s}$, for purposes of specifying the correct incoming amino proton on (a) syn- $\mathrm{A} 0^{0} 2$ \# for

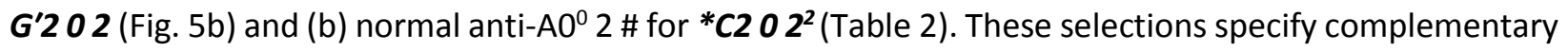
mispairs for the "in progress" $t s, G^{\prime} \mathbf{2} 0 \mathbf{2} \rightarrow T$ and ${ }^{*} \mathbf{C} \mathbf{2} \mathbf{O} \mathbf{2}^{\mathbf{2}} \rightarrow T$. Consequently, before physical incorporation of the $\boldsymbol{G}^{\prime} \rightarrow T$ or ${ }^{*} \boldsymbol{C} \rightarrow T$ substitution, "entangled" $\boldsymbol{G}^{\prime} \mathbf{2} \boldsymbol{O} \mathbf{2}$ (Fig. 4b) and "entangled" *C2 $0 \mathbf{2}^{\mathbf{2}}$ (Fig. 4c) are 
W. Grant Cooper; Evolution Via EPR-Entanglement Algorithm. Journal of Biomedical Engineering and Medical Imaging, Volume 4, No 2, April (2017), pp 43-95

deciphered and transcriptionally expressed by the E. coli host's RNA polymerase as normal T2 $02^{2}$ (Fig. 4a), as observed [10-11,81-82]. In these cases, entangled eigenstates, $\mathbf{G}^{\prime} \mathbf{2} \mathbf{0} \mathbf{2}$ and ${ }^{*} \mathbf{C} \mathbf{2} \mathbf{0} \mathbf{2}^{\mathbf{2}}$, are subjected to quantum transcriptase measurements, $\mathbf{G}^{\prime} \mathbf{2} \mathbf{0} \mathbf{2} \rightarrow T$ and ${ }^{*} \mathbf{C} \mathbf{2} \mathbf{0} \mathbf{2}^{\mathbf{2}} \rightarrow T$, and subsequently (or simultaneously), $100 \%$ of the transcribed, entangled eigenstates $-\mathbf{G}^{\prime} \mathbf{2} \mathbf{0} \mathbf{2}$ and ${ }^{*} \mathbf{C} \mathbf{2} \mathbf{0} \mathbf{2}^{\mathbf{2}}$ - participate in the entangled enzyme quantum searches, $\Delta \mathrm{t}^{\prime} \leq 10^{-14} \mathrm{~s}[1-3,6-8,10-11]$. This generates the identical frequencies of base substitutions, G'2 $02 \rightarrow T$ and ${ }^{*} \mathbf{C} \mathbf{2} \quad \mathbf{O} \mathbf{2}^{\mathbf{2}} \rightarrow T$, via quantum transcription before replication, and subsequently, expressible as decohered incorporated base substitutions (Table 2). This $100 \%$ efficiency of expressing $\mathbf{G}^{\prime} \mathbf{2} \mathbf{O} \mathbf{2} \rightarrow T$ and ${ }^{*} \mathbf{C} \mathbf{2} \mathbf{0} \mathbf{2}^{\mathbf{2}} \rightarrow T$-via quantum transcription before replication - at the identical frequencies exhibited by ultimately incorporated ts substitutions, is a consequence of the fact that the "pre-replication" quantum mechanically transcribed eigenstate-entanglement is subsequently a component in the replicated, decohered complementary mispair [1-3], created by the entangled enzyme quantum search, $\Delta \mathrm{t}^{\prime} \leq 10^{-14} \mathrm{~s}$ (Fig.5; Table 2). This generates a 2-fold "mutation enhancement" of $\boldsymbol{G}^{\prime} \rightarrow T$ and ${ }^{*} \boldsymbol{C} \rightarrow T$ substitutions [2,10-11], which explain the $65.5 \%$ A-T content of T4 phage DNA [79]. Evolutionary analyses [2] - that explain the relative
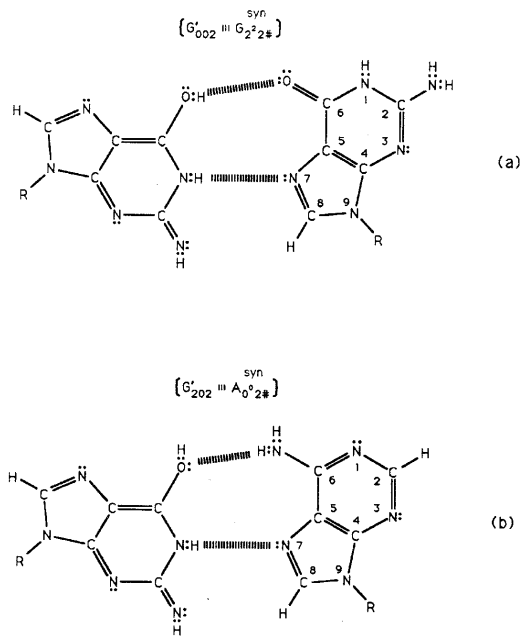

Figure 5

(Figure 5. Complementary transversion mispairs created by enzyme-proton-eigenstate entanglement executing a quantum search.)

distribution of the 22 most abundant microsatellites (STRs [20]) common to rat and human - require ts and $\boldsymbol{t} \boldsymbol{d}$ properties exhibited by T4 phage [6,9-11] to also be analogously operational in evolving rat and human genomes. Additionally, entangled enzyme quantum search times, $\Delta \mathrm{t}^{\prime} \leq 10^{-14} \mathrm{~s}[1-3,6,12,75]$, for specifying the complementary mispair exclude classical interactions [71] with ions, $\mathrm{H}_{2} \mathrm{O}$ and random temperature fluctuations. 


\subsection{Quantum Theoretical Treatment of "Nonclassical” Molecular Genetic Observables}

\subsubsection{Asymmetric Channel}

Hydrogen bonds in duplex DNA genomes are replicated into the metastable keto-amino state [1-4,6-11] where reduced energy, enol and imine proton qubit states are initially unoccupied, but are energetically accessible via EPR isomerization, keto-amino $\rightarrow$ enol-imine [13-16]. In the asymmetric case (Fig. $1 \mathrm{~b} \&$ Fig. 2f-g) of EPR-generated G-C $\rightarrow{ }^{*} G-{ }^{*} \boldsymbol{C}$, quantum uncertainty limits, $\Delta x \Delta p_{x} \geq \hbar / 2$, operate on hydrogen bonding amino $\left(-\mathrm{NH}_{2}\right)$ protons of cytosine, causing confinement of amino protons to too small of space, $\Delta x[59,93]$. This creates direct quantum mechanical proton - proton physical interaction, which generates the asymmetric EPR arrangement, keto-amino $\rightarrow$ enol-imine (Fig. 1b), where position and momentum entanglement is introduced between separating imine and enol protons. Molecular neutrality and stability of complementary ${ }^{*} \mathbf{G}-{ }^{*} \boldsymbol{C}$ quantum entanglement superposition states within the double helix are satisfied by an energetic amino cytosine proton projected between two identical sets of electron lonepairs on the complementary guanine keto oxygen, and transfer of the hydrogen bonded ring proton, from guanine to cytosine, and simultaneously, intramolecular reorganization of appropriate $\pi$ and $\sigma$ electrons, illustrated in Fig. 1b. Each reduced energy, entangled imine and enol product proton is shared between two indistinguishable sets of electron lone-pairs, and therefore, participates in entangled quantum oscillations $[2,6,10]$ at $\sim 10^{13} \mathrm{~s}^{-1}$, into and out of major $(\sim 22 \AA)$ and minor $(\sim 12 \AA)$ genome grooves [73-74], between near symmetric energy wells in decoherence-free subspaces [63-65]. This specifies quantum dynamics of an EPR pair of entangled enol and imine proton qubits until measured, $\delta \mathrm{t}<10^{-13} \mathrm{~s}$, by an enzyme quantum processor [12]. The imine and enol protons constitute an "entangled pair" of two-state proton qubits on opposite DNA strands.

An entangled enol or imine proton is in state $\mid+>$ when it is in position to participate in interstrand hydrogen bonding and is in state $\mid->$ when it is "outside", in a major or minor DNA groove [1-4,6-11]. The quantum mechanical state of the entangled pair of proton qubits can be viewed as a vector in the fourdimensional Hilbert space that describes the quantum position state of two protons. The most general quantum mechanical state of these two protons can be written as

$$
\left|\psi>=c_{++}\right|++>+c_{+-}\left|+->+c_{-+}\right|-+>+c_{--} \mid-->
$$

where the first symbol, + or -, represents proton 1 and the second symbol represents proton 2, and the expansion coefficients, $c^{\prime}$ s, satisfy normalization, $\left|c_{++}\right|^{2}+\left|c_{+-}\right|^{2}+\left|c_{-+}\right|^{2}+\left|c_{--}\right|^{2}=1$. Since Eq (1) cannot be expressed as a tensor product of protons 1 and 2, maximally entangled quantum states for the qubit pair of imine and enol protons can be written in terms of the four Bell [17-18] states, expressed as

$$
\begin{aligned}
& \left|\Phi^{+}\right\rangle=1 / \mathrm{v} 2\{|++>+|-->\} \\
& \mid \Phi^{-}>=1 / \mathrm{v} 2\{|++>-|-->\} \\
& \mid \phi^{+}>=1 / \mathrm{v} 2\{|+->+|-+>\} \\
& \mid \phi^{-}>=1 / \mathrm{v} 2\{|+->-|-+>\} .
\end{aligned}
$$


W. Grant Cooper; Evolution Via EPR-Entanglement Algorithm. Journal of Biomedical Engineering and Medical Imaging, Volume 4, No 2, April (2017), pp 43-95

\subsubsection{Symmetric Channel}

The symmetric keto-amino $\rightarrow$ enol-imine channel is initiated by quantum uncertainty limits, $\Delta x \Delta p_{x} \geq \hbar / 2$, initially operating on amino $\left(-\mathrm{NH}_{2}\right)$ protons of guanine carbon-2 (Fig. 1a). The energetic proton receives recoil energy resulting from proton-proton confinement to too small of space, $\Delta x$. This direct quantum mechanical proton - proton physical interaction between guanine carbon- 2 amino protons generates the initial EPR arrangement [13], keto-amino $\rightarrow$ enol-imine, where position and momentum entanglement is introduced between separating, carbon-2 side chain, enol and imine $\boldsymbol{G}^{\prime}-\boldsymbol{C}^{\prime}$ protons (Fig. 1a). This proton transfer initiates intramolecular reorganization of $\pi$ and $\sigma$ electrons within guanine and cytosine, which subjects "distorted" amino protons of cytosine to quantum uncertainty limits, $\Delta x \Delta p_{x} \geq \hbar / 2$, in too small of space, $\Delta x$. The resulting direct quantum mechanical proton - proton physical interaction generates the second EPR arrangement, keto-amino $\rightarrow$ enol-imine, where position - momentum entanglement is imposed between separating, carbon- 6 side chain imine and enol protons. In this case, the initial proton entanglement reaction induced the second proton separation entanglement reaction, thereby generating two sets of entangled proton "qubit pairs" as consequences of two sequential EPR arrangements, ketoamino $\rightarrow$ enol-imine [1-2,6]. Each of the four reduced energy enol and imine protons is shared between two indistinguishable sets of electron lone-pairs, and thus, participates in entangled quantum oscillations at $\sim 10^{13} \mathrm{~s}^{-1}$ between near symmetric energy wells in decoherence-free subspaces [63-65]. This specifies quantum dynamics for the two sets of entangled proton qubits occupying $\boldsymbol{G}^{\prime}-\boldsymbol{C}^{\prime}$ isomer pair superpositions, until measured by "Grover's-type" [1-3,12] quantum processors.

The dimensionality of the Hilbert space required to express the quantum mechanical state for four proton qubits is sixteen, i.e., $2^{\mathrm{N}}=2^{4}=16$. Each entangled imine and enol proton is shared between two sets of indistinguishable electron lone-pairs, and thus, participates in entangled quantum oscillations between near symmetric energy wells at $\sim 10^{13} \mathrm{~s}^{-1}$ in decoherence-free subspaces, which specifies entangled proton qubit dynamics occupying a heteroduplex heterozygote $\boldsymbol{G}^{\prime}-\boldsymbol{C}^{\prime}$ superposition site $[1-2,6,10-11,80]$. In this case, two sets of entangled imine and enol proton qubits - four protons constituting two sets of entangled "qubit pairs" - occupy the complementary $G^{\prime}-C^{\prime}$ superposition isomers such that enzyme quantum reader "measurement" of $\boldsymbol{G}^{\prime}$-protons specifies, instantaneously [13-16], quantum states of the four entangled qubits that occupy the sixteen-dimensional space.

Studies of heteroduplex heterozygote $\boldsymbol{G}^{\prime}-\boldsymbol{C}^{\prime}$ sites with $\boldsymbol{G}^{\prime}$ on the transcribed strand $[2,6-7,9-11,80]$ require the enzyme quantum reader to "measure", specify and execute quantum informational content of sixteen different entangled proton qubit $\mathbf{G}^{\prime}-\boldsymbol{C}^{\prime}$ states (Table 3). In the example of Fig. $2, \mathbf{G}^{\prime} \mathbf{0} \mathbf{0} \mathbf{2}\left(\mathbf{G}^{\prime} \mathbf{O} \mathbf{0} \mathbf{2} \rightarrow \mathbf{C}\right.$ ), the carbon-2 imine proton is in state $\mid->$ groove position, whereas the eigenstate $\mathbf{G}^{\prime} \mathbf{2} \mathbf{0} \mathbf{2}\left(\mathbf{G}^{\prime} \mathbf{2} \mathbf{0} \mathbf{2} \rightarrow T\right)$ has both carbon-2 imine and carbon- 6 enol protons in state $\mid->$ groove positions. Eigenstate $G^{\prime} \mathbf{2} 00\left(G^{\prime} \mathbf{2} 00\right.$ $\rightarrow G$; "null" mutation) has the carbon- 6 enol proton "trapped" in a state $\mid->$ DNA groove, but entangled enol and imine protons for eigenstate $\mathbf{G}^{\prime} \mathbf{0} \mathbf{0} \mathbf{0}$ are both in state $\mid+>$, the "interior" interstrand hydrogen bond position. Since the enol and imine quantum protons on $\boldsymbol{G}^{\prime}$ are one-half of the four entangled imine and enol $\boldsymbol{G}^{\prime}-\boldsymbol{C}^{\prime}$ proton qubit pairs, enzyme quantum reader measurements on $\boldsymbol{G}^{\prime}$-proton states specifically select quantum mechanical qubit states, $\mid->$ and $\mid+>$, for the four entangled $\boldsymbol{G}^{\prime}-\boldsymbol{C}^{\prime}$ protons. Here the entangled pair - guanine carbon-2 imine and cytosine carbon-2 enol - are identified, respectively, as protons numbers I and II (Roman numerals). Proton numbers III and IV, respectively, are cytosine carbon6 imine and guanine carbon- 6 enol. Using this notation, the enzyme quantum reader measures the four entangled proton qubit states of $\mathbf{G}^{\prime} \mathbf{O} \mathbf{0} \mathbf{2}$ as $\mid-+-+>$, i.e., guanine imine proton I is in state $\mid->$, cytosine 
enol proton II is in state $\mid+>$, cytosine imine proton III is in state $\mid->$, and guanine enol proton IV is in state $\mid+>$. Similarly, the measured proton qubit state for $G^{\prime} \mathbf{2} 0 \mathbf{2}$ is $\mid-++->$, and is $\mid+-+->$ for $\mathbf{G}^{\prime} \mathbf{2} \mathbf{0} \mathbf{0}$, and finally, is $\mid+--+>$ for eigenstate $\boldsymbol{G}^{\prime} \mathbf{0} \mathbf{0} \mathbf{0}$. In addition to the four quantum mechanical states of $\boldsymbol{G}^{\prime}$ imposed by enzyme quantum reader measurements (Fig. $2 \mathrm{~b}$-e), twelve additional states are required to specify the four two-state entangled proton qubits. The $\boldsymbol{G}^{\prime}-\boldsymbol{C}^{\prime}$ site superposition consist of two sets of intramolecular entangled proton qubit pairs that are participating in quantum oscillations between near symmetric energy wells in decoherence-free subspaces [1-2,10,63-65] at $\sim 10^{13} \mathrm{~s}^{-1} \mathrm{~s}$. Therefore, the most general quantum mechanical state of these four $\boldsymbol{G}^{\prime}-\boldsymbol{C}^{\prime}$ protons is given by

$$
\begin{aligned}
& \left|\Psi>=c_{1}\right|-+-+>+c_{2}\left|---+>+c_{3}\right|--++>+c_{4} \mid-+++> \\
& +c_{5}\left|-++->+c_{6}\right|---->+c_{7}\left|-+-->+c_{8}\right|--+-> \\
& \quad+c_{9}\left|+-+->+c_{10}\right|+++->+c_{11}\left|++-->+c_{12}\right|+--> \\
& \quad+c_{13}\left|+--+>+c_{14}\right|++++>+c_{15}\left|+-++>+c_{16}\right|++-+>,
\end{aligned}
$$

where the $c_{i}^{\prime}$ s represent, generally complex, expansion coefficients. Since the 16-state superposition of four entangled proton qubits occupy enol and imine "intra-atomic" subspaces, shared between two indistinguishable sets of electron lone-pairs, the entangled quantum superposition system will persist in evolutionarily selected decoherence-free subspaces [63-65,99] until an invasive perturbation, e.g., "measurement", exposes the previously "undisturbed" quantum mechanical superposition $[2,7,10$ $11,75]$. Just before enzyme quantum reader measurement of a $\boldsymbol{G}^{\prime}-\boldsymbol{C}^{\prime}$ site where $\boldsymbol{G}^{\prime}$ is on the transcribed strand, the 16 -state $\boldsymbol{G}^{\prime}-\boldsymbol{C}^{\prime}$ superposition system is described by Eq (6). In an interval $\delta \mathrm{t} \ll 10^{-13} \mathrm{~s}$, the enzyme quantum reader simultaneously detects entangled $\boldsymbol{G}^{\prime}$-protons I (carbon-2 imine) and IV (carbon6 enol) in either correlated position states, $|-\rangle$ or $|+\rangle$, which are components of an entangled proton "qubit pair". When proton I or IV is measured by the quantum reader in position state, $\mid->$ or $\mid+>$, the other member of this entangled pair will, instantaneously [13-16], be in the appropriately correlated state, $|+\rangle$ or $|-\rangle$, respectively. Protons detected in state $|-\rangle$, "outside" groove position, form "new" entanglement states with the proximal quantum reader $[2,12]$ that enable enzyme quantum coherence to implement its quantum search, $\Delta \mathrm{t}^{\prime} \leq 10^{-14} \mathrm{~s}$, which specifies an incoming electron lone-pair, or amino proton, belonging to the tautomer selected for creating the "correct" complementary mispair (Fig. 5). Protons detected in state $|+\rangle$, "inside" hydrogen bonding position, contribute to specificity of the $\boldsymbol{G}^{\prime}$ genetic code, exemplified by both $\mathbf{G}^{\prime} \mathbf{2} 0 \mathbf{2}$ and *C20 $\mathbf{2}^{\mathbf{2}}$ "measured as" normal T2 $2^{2} 2^{2}$ (Fig. 4) via quantum transcription and replication [10-11]. Since the quantum reader detects entangled $\boldsymbol{G}^{\prime}$-protons I and IV in states $\mid->$ or $\mid+>$, the "matching" correlated quantum states, $\mid+>$ or $\mid->$, of entangled $\boldsymbol{C}^{\prime}$-protons II and III were instantaneously specified. Consequently, enzyme quantum reader "measurement" on $\boldsymbol{G}^{\prime}$-protons I and IV converts, instantaneously, the 16-state quantum system of Eq (6) into the 4-state system $\dot{c}_{1}\left|-+-+>, \dot{c}_{5}\right|-++->, \dot{c}_{9}\left|+-+->, \dot{c}_{13}\right|+--+>-$ listed in column B of Table 3 and illustrated in

\{Table 3. Evolution of the sixteen-state entangled proton qubit $\mathrm{G}^{\prime}-\mathrm{C}^{\prime}$ superposition, before measurement (column A), after measurement, $\Delta \mathrm{t}^{\prime} \leq 10^{-14} \mathrm{~s}$ (column B), and decohered observables (column D).\} 
Unperturbed (A) and instantaneous yield of "measured” (B) G'-C' entangled proton qubit states, showing results of entangled enzyme quantum search, $\Delta \mathrm{t}^{\prime} \leq 10^{-14} \mathrm{~s}$, (C) and molecular clock (D) results, $t$.

\begin{tabular}{|c|c|c|c|}
\hline A & B & $\mathrm{C}$ & D \\
\hline$\left.c_{I}\right|^{*-+-+>}$ & $c_{l} \mid-+-+>$ & $\operatorname{syn}-G 2^{2} 2 \#$ & $G^{\prime} 002 \rightarrow \mathrm{C}$ \\
\hline$\left.c_{2}\right|^{*}---+>$ & $\left.c_{2}\right|^{-+-+>}$ & & \\
\hline$\left.C_{3}\right|^{*--++>}$ & $\left.c_{3}\right|_{-+-+>}$ & & \\
\hline$\left.c_{4}\right|^{*-+++>}$ & $\left.c_{4}\right|^{-+-+>}$ & & \\
\hline$\left.c s\right|^{*-++*->}$ & $c s \mid-++->$ & $\operatorname{syn}-\mathrm{A} 0^{\circ} 2 \#$ & $G^{\prime} 202 \rightarrow \mathrm{T}$ \\
\hline$\left.c_{s}\right|^{*-\ldots} *->$ & $\left.c_{s}\right|^{-++}>$ & & \\
\hline$\left.c_{7}\right|^{*-++-_{-}>}$ & $\left.c_{7}\right|^{-++}>$ & & \\
\hline$\left.C_{3}\right|^{*--}++_{->}$ & $\left.c_{s}\right|^{-++}>$ & & \\
\hline$c_{9} \mid+-+* \rightarrow$ & $\left.c_{9}\right|_{++} ^{+}$ & $\mathrm{CO}^{\circ} 22^{2}$ & $G^{\prime} 200 \rightarrow \mathrm{G}$ \\
\hline$\left.C_{10}\right|_{+++* \rightarrow}$ & $\mathrm{ClO}_{20}+++-$ & & \\
\hline$c_{n} \mid++-*->$ & $c_{n} \mid++->$ & & \\
\hline$c_{12} \mid+-*_{->}$ & $c_{n 2}+++>$ & & \\
\hline$c_{I 3} \mid+-+>$ & $c_{I z} \mid++>$ & none & $G^{\prime} 000 \rightarrow ?$ \\
\hline$\left.c_{I 4}\right|_{++++>}$ & $c_{I 4} \mid++>$ & & $?=$ microcolony \\
\hline$c_{I S} \mid+-++>$ & $c_{I S} \mid+-+>$ & & \\
\hline$c_{I G} \mid++-+>$ & $c_{I G} \mid+-+>$ & & \\
\hline
\end{tabular}

Fig. $2 \mathrm{~b}$-e, where expansion coefficients, $\dot{c}_{i}$, are defined by $\dot{c}_{1}=\Sigma^{4}{ }_{\mathrm{i}=1} c_{i}, \dot{c}_{5}=\Sigma^{8}{ }_{\mathrm{i}}={ }_{5} c_{i}, \dot{c}_{9}=\Sigma^{12}{ }_{\mathrm{i}}=9 c_{i}$, and $\dot{c}_{13}=$ $\Sigma \Sigma_{i=13}^{16} c_{i}$. This result is displayed in Table 3 where column $A$ identifies the unperturbed 16-state quantum system of Eq (6). Column B contains the distribution of $\mid->$ and $\mid+>$ proton states - for $\boldsymbol{G}^{\prime}-\boldsymbol{C}^{\prime}$ protons: I, II, III, IV - generated instantaneously as a consequence of the quantum reader initially "measuring" quantum states of entangled $\boldsymbol{G}^{\prime}$-protons I and IV. The instantaneously generated quantum states $-\dot{c}_{1}\left|-+-+>, \dot{c}_{5}\right|-++->, \dot{c}_{9}\left|+-+->, \dot{c}_{13}\right|+--+>-$ provide, instantaneously, specific instructions for the enzyme - proton entanglement before it embarks on its entangled enzyme "quantum 
quest", $\Delta \mathrm{t}^{\prime} \leq 10^{-14} \mathrm{~s}$, of selecting the particular incoming tautomer specified by molecular evolution, $t s$ requirements [1-2,6-8]. Incoming tautomers selected by entangled enzyme quantum searches are identified in column $\mathrm{C}$ and resultant molecular clock substitutions, $t$, are listed in column D of Table 3.

In intervals, $\delta \mathrm{t} \ll 10^{-13} \mathrm{~s}$, the enzyme quantum processor measurement apparatus "traps" entangled $\boldsymbol{G}^{\prime}$ imine and/or enol protons - I and IV - in DNA grooves, specified by state $\mid->$, and consequently, the position state, $\mid->$ or $\mid+>$, is instantaneously specified for the four entangled $\mathbf{G}^{\prime}$ - $\mathbf{C}^{\prime}$ protons: I, IV and II, III. In column A of Table 3, an entanglement state between the quantum reader and a "groove" proton is indicated by superscript, “*”, e.g., | ${ }^{*-+-+>}$, identifying $G^{\prime}$ proton I as the enzyme - entangled "groove" proton. The "new" entanglement state between the quantum reader and the "trapped" proton enables enzyme quantum coherence to be immediately exploited in implementing an entangled enzyme quantum search, $\Delta \mathrm{t}^{\prime} \leq 10^{-14} \mathrm{~s}$, which ultimately specifies the particular $\mathbf{t s}$ as $\mathbf{G}^{\prime} \mathbf{0} \mathbf{0} \mathbf{2} \rightarrow C, \mathbf{G}^{\prime} \mathbf{2} \mathbf{0} \mathbf{2} \rightarrow T$ or $\mathbf{G}^{\prime} \mathbf{2} \mathbf{0} \mathbf{0} \rightarrow \mathbf{G}$ $[2,6,10-11]$. The specificity of each $t s$ is governed by the entangled enzyme quantum search selecting the correct incoming tautomers - syn-G2 2 \#, syn- $\mathrm{AO}^{0} 2 \#, \mathrm{CO}^{0} 22^{2}$ - respectively, for proton qubit eigenstates - G'O 0 2, G'2 0 2, G'2 00 - illustrated in Fig. 4, Table 2 and Table 3. Natural selection has exploited quantum entanglement properties of proton qubits [60-62], which allow enzyme - proton entanglement to specify and implement results of an entangled enzyme quantum search in intervals, $\Delta \mathrm{t}^{\prime} \leq 10^{-14} \mathrm{~s}$ [1$2,6,10-12]$. This mechanism implies that enzyme - proton entanglement implementation of an enzyme quantum search would not be successful without instantaneous specification [13-16] of the four $\mathbf{G}^{\prime}-\boldsymbol{C}^{\prime}$ entangled proton qubit states determined by quantum reader "measurements" on the two $\boldsymbol{G}^{\prime}$-proton qubits, I and IV, associated with the transcribed strand (Table 3).

\subsection{Enzyme - Proton Entanglement-Enabling Grover's [12] Quantum Search for "incoming" Tautomer}

The enzyme quantum reader "measurement apparatus" patrols the double helix along major ( $22 \AA$ ) and minor ( $12 \AA$ ) grooves [64,73-74], creating entanglement states between "measured" enol and imine entangled qubit "groove protons" and proximal enzyme components [1-3,12]. The quantum reader polymerase energy source is ATP, and it maintains a reservoir of purines, pyrimidines and nucleotides for base pairing operations. Davies [100] has noted that the polymerase protein has a mass of about $10^{-19} \mathrm{~g}$, and a length of about $10^{-3} \mathrm{~cm}$ and travels at a speed of about $100 \mathrm{bp}$ per sec., or about $10^{-5} \mathrm{~cm} \mathrm{~s}^{-1}[58,101]$. Curiously, the normal speed of the polymerase, $\sim 10^{-5} \mathrm{~cm} \mathrm{~s}^{-1}$, corresponds to the limiting speed allowed by the energy-time uncertainty relation for the operation of a quantum clock. For a clock of mass $m$ and size $l$, Wigner [102] found the relation

$$
T<m l^{2} / \hbar
$$

Equation (7) can be expressed in terms of a velocity inequality given by

$$
\mathbf{v}>\hbar / m l \text {, }
$$

which, for this polymerase, yields a minimum velocity of about $10^{-5} \mathrm{~cm} \mathrm{~s}^{-1}$, implying the quantum reader enzyme speed of operation can be confined by a form of quantum synchronization uncertainty [100]. The quantum reader "measurement apparatus" has been evolutionarily selected to decipher, process and accurately exploit informational content within DNA base pairs composed of either $(a)$ the classical keto- 
W. Grant Cooper; Evolution Via EPR-Entanglement Algorithm. Journal of Biomedical Engineering and Medical Imaging, Volume 4, No 2, April (2017), pp 43-95

amino state, $(b)$ undisturbed, enol and imine entangled proton qubit states - Eqs $(2-6)$ - including enzyme - proton entanglements participating in an entangled enzyme quantum search, $\Delta \mathrm{t}^{\prime} \leq 10^{-14} \mathrm{~s}[1-$ $4,6,12,75]$.

The enzyme quantum measurement-operator is identified by $M$, and operates on $\boldsymbol{G}^{\prime}$-proton states located on the transcribed strand to yield three different entanglement states between groove protons and enzyme components. From column B of Table 3, these enzymatic quantum "measurements", and resulting enzyme-proton entanglements, can be symbolically represented by

$$
\begin{aligned}
& M\left|-+-+>=\dot{c}_{1}\right|-+-+>\hat{E} p_{1} \\
& M\left|-++->=\dot{c}_{5}\right|-++->\hat{E} p_{1,} p_{\mathrm{IV}} \\
& M\left|+-+->=\dot{c}_{9}\right|+-+->\hat{E} p_{I V}
\end{aligned}
$$

where $\hat{E} p_{\mathrm{I}}, p_{\mathrm{IV}}$ in $\mathrm{Eq}(10)$ represents quantum entanglement between "groove" proton I (G'2 02 2-imine) and "groove" proton IV (G'2 0 2-enol) and proximal enzyme components. Similarly, $\hat{E} p_{I}$ and $\hat{E} p_{I V}$, represent alternative entanglements between enzyme components and entangled proton I, and separately, entangled proton IV, respectively. The original unperturbed groove proton's "quantumness" becomes distributed over an enzyme "entanglement site", which is selected to complete its assignment of specifying the complementary mispair before proton decoherence, i.e., $\Delta \mathrm{t}^{\prime}<\tau_{\mathrm{D}}<10^{-13} \mathrm{~s}[1-2,6-8]$. Each of the three enzyme-proton entanglements implements a different "selective" quantum search, $\Delta \mathrm{t}^{\prime} \leq 10^{-14} \mathrm{~s}$ $[1-3,12,75]$, to specify the correct evolutionarily required purine or pyrimidine tautomer to properly complete the molecular clock [6,9-11] base substitution, $t$, by a quantum processing [1-4,12], TopalFresco [9-11,92] substitution-replication mechanism (Table 2; Fig. 5). Since quantum informational content is deciphered by enzymatic processing of entangled proton qubits shared between two indistinguishable sets of electron lone-pairs, the entangled enzyme quantum search mechanism is assumed to initially select the incoming tautomer on the basis of electron lone-pair, or amino proton, availability. Evidently the "evolved" quantum reader has an immediately accessible "reservoir" of required tautomers for quantum search selection [1-4,9-11].

Evidence discussed here [1-12,29,63-65,67-70,80-82,97] implies a "Grover's-type" enzyme-entanglement complex has been evolutionarily selected and refined over the past $~ 3.5$ or so billion y to implement sets of entangled enzyme quantum searches. In this model of molecular genomic evolution, an evolutionarily selected enzyme-proton entanglement implements a quantum search of the evolutionarily available purine and pyrimidine database for the "matching" classical tautomer required to execute an "in progress" complementary mispair formation before proton decoherence $[1-4,75]$. The initial component of the complementary mispair - the particular eigenstate - was selected by "new" quantum entanglement between the "trapped" entangled groove proton and the Grover's enzyme quantum reader. The enzyme - proton entanglement implements a quantum search which specifies - in intervals, $\Delta \mathrm{t}^{\prime} \leq 10^{-14} \mathrm{~s}[1-4,12]$ - the incoming electron lone-pair, or amino proton, belonging to the tautomer required to create the complementary mispair (Fig.5; Table 2; Table 3). This allowed quantum coherence of the entangled 
ribozyme and/or enzyme to specify the particular ts or $\boldsymbol{t} \boldsymbol{d}$, and thus, enable entanglement-directed genomic evolution.

When both imine and enol $\boldsymbol{G}^{\prime}$-protons occupy groove positions at time of measurement, the enzymeproton entanglements specify eigenstate, $\mathbf{G}^{\prime} \mathbf{2} \mathbf{0} \mathbf{2}$. In this case, the enzyme quantum-reader imposes "new" entanglement conditions on the two G'2 02 groove protons, I and IV (Fig. 4b). This creates the proton-enzyme entanglement, i.e., $\hat{E} p_{\mathrm{I}}, p_{\mathrm{IV}}$ in Eq (10), and "simultaneously", an output informational transcription qubit, $\mathbf{G}^{\prime} \mathbf{2} \mathbf{0 2} \rightarrow T$, is generated. The resulting enzyme-proton-eigenstate entanglement will execute its evolutionarily specified assignment of identifying syn- $\mathrm{AO}^{\circ} 2$ \# to create the designated

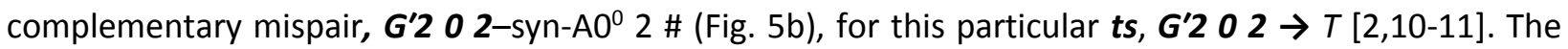
entangled protons will retain coherence until specification of the complementary mispair, i.e., G'2 0 2-

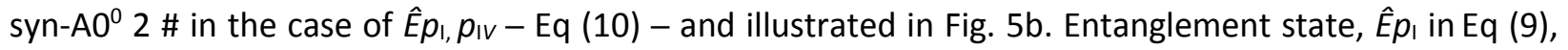

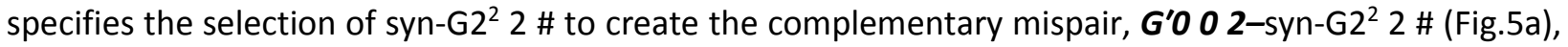
which generates the transversion substitution, $\mathbf{G}^{\prime} \mathbf{0} 02 \rightarrow C[34,43]$. Entanglement $\hat{E} p_{I V}$ in Eq (11) selects normal $\mathrm{CO}^{0} 22^{2}$ (Table 2), which yields a detectable, T4 phage plaque, "null" substitution [9-11,80], G'2 0 $0 \rightarrow \mathrm{G}^{2} \mathrm{O} 0^{0}$.

The enzyme quantum measurement apparatus functions as a linear operator acting on states of entangled proton qubits oscillating into, and out of, DNA grooves at $\sim 10^{13} \mathrm{~s}^{-1}$. This allows creation of enzyme-protoneigenstate entanglements $-G^{\prime} \mathbf{2} 0 \mathbf{2}, \mathbf{G}^{\prime} \mathbf{O} \mathbf{0} \mathbf{2}, \mathbf{G}^{\prime} \mathbf{2} \mathbf{0} \mathbf{0}$ - that select the particular classical isomers, i.e., syn$A 0^{0} 2$ \#, syn-G2 2 \# and $\mathrm{CO}^{\circ} 22^{2}$ (Tables 2-3; Fig. 5), respectively, for the formation of complementary mispairs. Since these complementary mispairs are specified in intervals, $\Delta \mathrm{t}^{\prime} \leq 10^{-14} \mathrm{~s}$, the relevant classical isomer components are immediately accessible. When the selected classical isomer is interfaced with its corresponding entangled eigenstate, the linear superposition system collapses onto the eigenstate specified by the enzyme-entangled proton, which completes the "measurement" of the superposition base pair system [1-4,75-76]. These evolutionarily refined quantum search processes allow this enzymeproton entanglement to complete its task in intervals, $\Delta \mathrm{t}^{\prime} \leq 10^{-14} \mathrm{~s}[1-4,12]$, which is $\sim 10^{5}$-fold faster than classical expectations. However, Tegmark's [75] assessments of proton decoherence times imply the relation, $\Delta \mathrm{t}^{\prime}<\sim 10^{-15} \mathrm{~s}$.

\subsection{Origin of the Genetic Code Model}

According to the quantum information processing model [1-4], entangled proton qubit resources were initially introduced into ancestral duplex "RNA-like" segments associated with ribozymes [67-70,103]. RNA - ribozyme duplex nucleic acid components are assumed to have been composed of analogs of G - 5HMC (5hydroxymethylcytosine) and A-U [3]. Survival of ribozyme - RNA duplex components - populated with entangled proton qubits - required selection of rudimentary quantum bio-processors [2,12], operating on entangled proton qubits, creating peptide - ribozyme - proton RNA entanglements. Since quantum bioprocessors "measure" quantum informational content by selecting entangled proton qubit states, in intervals $\delta \mathrm{t}<<10^{-13} \mathrm{~s}[1-4,10]$, quantum reader operations can be approximated by a "truncated" Grover's [12] quantum search of "susceptible" unsorted entangled qubits occupying $\boldsymbol{G}^{\prime}-\mathbf{5} \boldsymbol{H} \mathbf{M C}$ ' and ${ }^{*} \boldsymbol{G}-\mathbf{5} \boldsymbol{H} \mathbf{M}^{*} \boldsymbol{C}$ superposition sites. Grover's algorithm is applicable for large system sizes $N$ in high dimensional Hilbert spaces where the quantum enabled database is unsorted. However, a quantum bio-processor searching a particular unsorted database of $N$ qubit states (here $N=20$ qubit states occupying $\boldsymbol{G}^{\prime}-\boldsymbol{C}^{\prime}+{ }^{*} \boldsymbol{G}-{ }^{*} \boldsymbol{C}$ sites; Eqs (2-6)) can be approximated by iterations of a "truncated" Grover's quantum search. The quantum bio- 
W. Grant Cooper; Evolution Via EPR-Entanglement Algorithm. Journal of Biomedical Engineering and Medical Imaging, Volume 4, No 2, April (2017), pp 43-95

processor is designed to identify entangled proton qubit states, including those occupying a RNA groove, where the "measurement" interval satisfies, $\delta \mathrm{t} \ll<10^{-13} \mathrm{~s}$. The quantum bio-processor peptide-ribozyme forms an entanglement state with the "trapped" proton that, before proton decoherence, $\tau_{D}<10^{-13} s,(a)$ generates quantum transcription information from "measured" entangled proton qubit states [2-3], e.g., $G^{\prime} \mathbf{2} 02 \rightarrow U, 5 H M C^{\prime} \mathbf{2} 02^{2} \rightarrow U$, etc, $(b)$ implements a "new" peptide bond between an "incoming" selected amino acid and an existing "in place" amino acid, and $(c)$ implements selection of an "incoming" tautomer to "pair with" the ultimately decohered eigenstate, specified by the "trapped" proton in a genome groove. In this context, quantum bio-processor operations can be qualitatively approximated by a "truncated" Grover's [12] algorithm. This approximation of a quantum bio-processor measurement on entangled proton qubit states occupying $\mathbf{G}^{\prime}-\mathbf{5} \mathbf{H} \mathbf{M} \mathbf{C}^{\prime}$ and ${ }^{*} \mathbf{G}-\mathbf{5} \mathbf{H} \mathbf{M}^{*} \boldsymbol{C}$ superpositions implies a "truncated" $(N=20$ qubit states) Grover's algorithm would yield an improved efficiency of $\sqrt{ } N$ over a classical search. If $J$ is the total number of bio-molecular quantum reader measuring operations, Grover's "truncated" algorithm states

$$
(2 \mathrm{~J}+1) \arcsin (1 / \mathrm{V} N)=\pi / 2,
$$

which yields the interesting solutions,

$$
\begin{array}{ll}
\mathrm{J}=1, & N=4 \\
\mathrm{~J}=2, & N=10.4 \\
\mathrm{~J}=3, & N=20.2 \\
\mathrm{~J}=4, & N=33.2 .
\end{array}
$$

Consistent with observables exhibited by T4 phage DNA, the model outlined here assumes quantum reader measurements of $\mathbf{G}^{\prime}-\mathbf{5 H} \mathbf{M C}^{\prime}$ and ${ }^{*} \mathbf{G}-\mathbf{5} \mathbf{H} \mathbf{M}^{*} \mathbf{C}$ superpositions generated RNA "transcription qubits" (Table 2) - G'2 $02 \rightarrow U, G^{\prime} \mathbf{2} 00 \rightarrow G, 5 H M^{*} C 202^{2} \rightarrow U,{ }^{*} G 020^{\circ} \rightarrow A-$ that provided single base RNA informational units as precursor mRNA and precursor tRNA. Measurements [10-11] imply that ${ }^{*} \mathbf{C 2} \mathbf{0} \mathbf{2}^{\mathbf{2}} \rightarrow$ $T$ yields ${ }^{*} \mathbf{G O} 2 \mathbf{O}^{\circ} \rightarrow A(\sim 100 \%)$ in the complementary strand. Precursor tRNA components were evidently retained in the bio-molecular quantum processor's "hard drive" reservoir until a sufficient "sampling" of entangled qubit states had been subjected to the particular set of measurements. In this case, the number of measurement operations, J, converged to a value that yielded adequate statistics. According to this qualitative model, the quantum entanglement algorithm, implemented by ribozyme - peptide quantum reader-processors, converged via natural selection, to three measurement operations - J = 3 in Eq (15) to obtain adequate statistical probabilistic measurements of 20 entangled proton qubit states occupying $\boldsymbol{G}^{\prime}-\mathbf{5 H} \boldsymbol{M} \boldsymbol{C}^{\prime}$ and ${ }^{*} \boldsymbol{G}-\mathbf{5 H} \mathbf{M}^{*} \boldsymbol{C}$ superposition sites; ${ }^{*} \boldsymbol{A}-{ }^{*} \boldsymbol{U}$ sites were deleted [11]. The three selected quantum processor measurements identified a triplet code for a precursor tRNA, where L-amino acids were selected. Three separate probabilistic measurement operations would "quantify" a sufficient number of the 20 different entangled proton qubit states, and also, specify about 20, i.e., 22, amino acids for participation in protein structure [103]. The scenario outlined here implies quantum reader measurements of entangled proton qubits occupying ancestral $\mathbf{G}^{\prime}-\mathbf{5 H} \mathbf{H} \mathbf{C}^{\prime},{ }^{*} \mathbf{G}-\mathbf{5 H} \mathbf{H}^{*} \boldsymbol{C}$ and ${ }^{*} \boldsymbol{A}-{ }^{*} \boldsymbol{U}$ superposition sites may have provided the initial quantum informational content, specifying evolutionary parameters for origin of the genetic code, consisting of $\sim 22 \mathrm{~L}$-amino acids specified by $4^{3}$ triplet codons. Such "fundamental" quantum entanglement evolutionary processes [1-4] may have been operationally retained in "evolved" eukaryote systems, and thus, appear to participate in expressing measurements of 
entangled proton qubits required to exhibit molecular evolution of STRs $[1-3,20]$, discussed in the following.

\section{Human - Rat STR Evolution in terms of Quantum Entanglement Algorithm}

\subsection{Initiation and Termination Codons via Grover's measurements of EPR- Generated Entangled Proton Qubits}

Observations [9-11,29] and analyses [1-4,6-8] imply metastable hydrogen bonding amino $\left(-\mathrm{NH}_{2}\right)$ protons encounter quantum uncertainty limits, $\Delta x \Delta p_{x} \geq \hbar / 2$, which generate probabilities of EPR-created entangled proton qubits [1-3,13]. Replication and transcription of entangled proton qubit superposition $\boldsymbol{G}^{\prime}-\boldsymbol{C}^{\prime}$ and ${ }^{*} \boldsymbol{G}-{ }^{*} \boldsymbol{C}$ sites yield observable time-dependent molecular clock base substitutions, $\boldsymbol{t} \mathbf{s}[6,10-11]-$

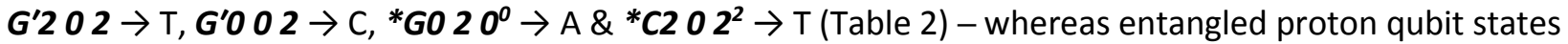
within ${ }^{*} \boldsymbol{A}-{ }^{*} \boldsymbol{T}$ sites, i.e., A-T $\rightarrow{ }^{*} \boldsymbol{A}-{ }^{*} \boldsymbol{T}$ (Fig. 3), exhibit time-dependent deletions, $\boldsymbol{t} \boldsymbol{d},{ }^{*} \boldsymbol{A} \rightarrow$ deletion and ${ }^{*} \boldsymbol{T}$ $\rightarrow$ deletion [11]. Also when $\boldsymbol{G}^{\prime}$ and/or ${ }^{*} \boldsymbol{C}$ is located on the transcribed strand, time-dependent substitutions, $\boldsymbol{t s}-\mathbf{G}^{\prime} \mathbf{2} \mathbf{O} \mathbf{2} \rightarrow \mathrm{T}$ and/or ${ }^{*} \mathbf{C} \mathbf{2} \mathbf{O} \mathbf{2}^{\mathbf{2}} \rightarrow \mathrm{T}$ - are expressed by "Grover's-type" transcriptase measurements of entangled proton qubits before replication is initiated (Fig. 4) [1-3,9-11,81-82]. Subsequent replication - after entangled enzyme quantum searches, $\Delta t^{\prime} \leq 10^{-14} \mathrm{~s}$ - expresses genotypically incorporated $\boldsymbol{t} \mathbf{s}-\mathbf{G}^{\prime} \mathbf{2} \mathbf{0 2} \rightarrow T$ and ${ }^{*} \mathbf{C} \mathbf{2} \mathbf{0} \mathbf{2}^{\mathbf{2}} \rightarrow T$ - at frequencies identical to those previously exhibited by quantum transcription before replication [9-11,81-82]. In these cases, $\boldsymbol{G}^{\prime} \rightarrow \mathrm{T}$ and ${ }^{*} \boldsymbol{C} \rightarrow \mathrm{T}$ contributions to the "gene pool" are 2-fold > "replication only" expectations [2,9-11], and transcriptase quantum processing specifies frequencies of subsequently incorporated $\boldsymbol{t} \boldsymbol{s}, \boldsymbol{G}^{\prime} \rightarrow \mathrm{T}$ and ${ }^{*} \boldsymbol{C} \rightarrow \mathrm{T}$.

Based on predictions of quantum entanglement algorithmic processing of EPR-generated entangled proton qubits accumulating with time in metastable duplex DNA base pairs, observed as G-C $\rightarrow G^{\prime}-C^{\prime}$, G$C \rightarrow{ }^{*} G-C^{*}$ and A-T $\rightarrow{ }^{*} A-{ }^{*} \boldsymbol{T}$ [9-11], the potential for a microsatellite [20] to exhibit expansion or contraction over evolutionary times can be qualitatively specified [1-2,6-7,29]. This hypothesis - based on observation [1-2,29] - assumes that the evolutionarily selected quantum entanglement algorithm responsible for $\boldsymbol{t} \boldsymbol{s}[1-4,6-8]$ and $\boldsymbol{t} \boldsymbol{d}[10-11]$ has been operational since the era of ancestral RNA - protein genomes [1-3,67-70,103], and therefore, has provided a source of time-dependent, 'point' genetic variation in all subsequently evolved duplex DNA [1-3,58]. The model also assumes a functional relationship exists between the relative positions of entangled proton qubit states within microsatellites and initiation regions for DNA replication [104]. Consequently, a time-dependent introduction of additional initiation codons - UUG, CUG, AUG, GUG - could cause the creation of additional polypeptides, some of which could be responsible for initiation of, or reinitiating, DNA synthesis [1-4,6-8,29]. Such additional initiating polypeptides could be responsible for adding more repeat units to an original microsatellite. Similarly, a time-dependent accumulation of stop codons - UAA, UAG, UGA - could introduce terminations of peptide chains that participate in transcription and replication. Subsequent transcription and resulting DNA synthesis would accordingly be altered, which could yield contractions exhibited by microsatellites [1-2,6-7,78]. An accumulation of entangled proton qubit states and subsequent transcriptase measurements [9-11] could specify the implementation of initiation codons and deletions or stop codons in microsatellites and/or their flanking sequences. Given observations [9-11,8082] consistent with the selected quantum entanglement algorithm for EPR-generated [13-18] time- 
W. Grant Cooper; Evolution Via EPR-Entanglement Algorithm. Journal of Biomedical Engineering and Medical Imaging, Volume 4, No 2, April (2017), pp 43-95

dependent molecular clock DNA evolution [1-4], the model - if applicable - should predict, qualitatively, the evolutionary distribution of the 22 most abundant microsatellites (Table 1) common to rat and human DNA $[2,20]$.

Although classical modes of evolution responsible for individual microsatellite length and their relative distribution throughout eukaryotic and prokaryotic genomes have remained an enigma [23,105-106], quantum entanglement algorithm processes [1-4,6-8] provide a rationale for relative expansion and/or contraction of a particular STR over evolutionary times [1-3,6-7,29]. Microsatellite duplexes whose "processed" [3,12] entangled proton qubits generate a preponderance of initiation codons - UUG, AUG, CUG, GUG - participate in the expansion mode of DNA synthesis [1-2,6,29], but if more termination codons - UAA, UGA, UAG - were introduced and/or the particular sequence consisted exclusively of A-T, such microsatellites would generally decrease in relative abundance over evolutionary times [1-2,7,1011 ]. This model is tested by comparing quantum entanglement algorithm predictions of microsatellite expansion or contraction with observation for each of the 22 most abundant microsatellites common to human and rat (Table 1). Analyses assumptions are (a) STR evolution is a consequence of EPR-generated entangled proton qubits populating STRs - or its flanking sequence - that is operated on by quantum entanglement algorithmic processes, which generates molecular clock events, $\boldsymbol{t}$ and $\boldsymbol{t} \boldsymbol{d}$, and their "dynamic" consequences [1-3,6-7,29,78], and (b) the rat genome is more ancient than human [107]. Results should provide an evolutionary rationale for the relative distribution of the 22 most abundant STRs in rat and human genomes [1-3,20].

\section{riplet Repeat and Contraction Unstable $(C C G)_{n}$ and $(C A G)_{n}$ Microsatellites}

Unstable repeat nucleotide sequences are responsible for $\sim 20$ or so heritable human genetic diseases [12,6, 29-34] and have been studied at the molecular level since 1991 [28]. However, internally consistent mechanisms responsible for microsatellite repeat, intergenerational instabilities [41-44,78] and subsequent expressions of diseases have been an enigma [46,108-109]. Insight into microsatellite instabilities is implied by consequences of the quantum entanglement algorithm operating on, for example, $(C C G)_{n}$ and $(C A G)_{n}$ microsatellites listed in Table 1. In cases of Fragile $X$ syndrome (FX), triplet repeats, $(C C G)_{n}$, are located in the 5 '-UTR of gene FMR1 where data indicate a maternal bias and a high upper limit expansion copy number of $\sim 2000$ (CCG) $)_{n}$ repeats [28-29]. Figure 6a illustrates that entangled proton qubit states in $(C C G)_{n}$ repeats would not generate stop codons, but three of the twelve ts pathways (25\%) could introduce entangled proton qubit states that could be measured to express an initiation codon, 5'-CUG-3'. Specifically, entangled proton qubits could accumulate for years to decades in oocyte DNA [110] before the enzyme quantum reader would "measure" 5'-C*C202 ${ }^{2}$ G-3' and 5'-CG'202G-3' entangled qubits, thereby expressing CUG via transcription of entangled proton qubit states (Fig. 4) and subsequent replication. Since the rate of accumulating entangled proton qubit states in haploid DNA would be smaller in $\sim 34{ }^{\circ} \mathrm{C}$ sperm [111] than in $37{ }^{\circ} \mathrm{C}$ oocyte genomes $[2,110]$, hyperexpansions (copy no. $>1000)$ of $(C C G)_{n}$ in oocyte 


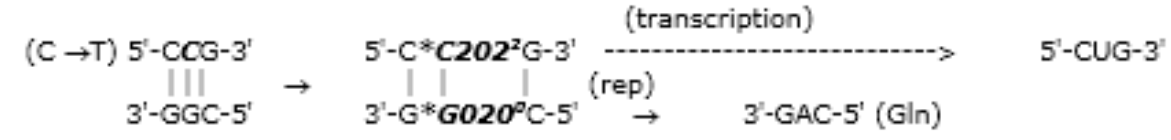

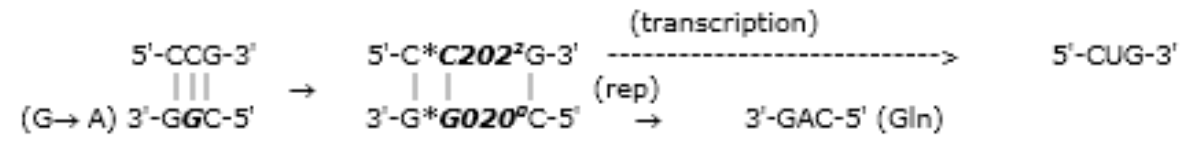

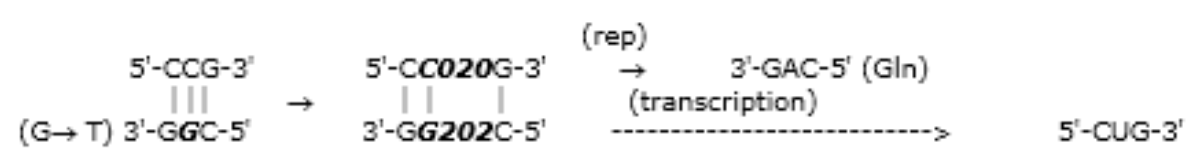

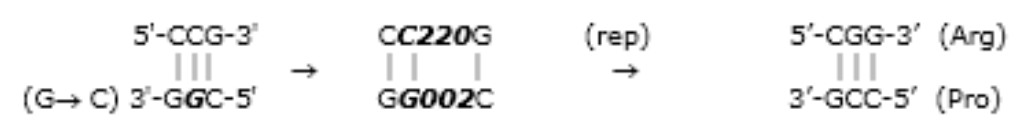

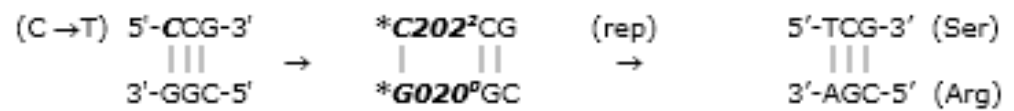

$$
\begin{aligned}
& (\mathrm{G} \rightarrow \mathrm{C}) 5^{\prime}-\mathrm{CCG}-3^{\prime} \quad \mathrm{CCGOO2} \quad(\mathrm{rep}) \quad 5^{\prime}-\mathrm{CCC}-3^{\prime} \text { (Pro) }
\end{aligned}
$$

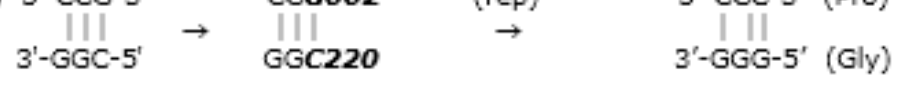

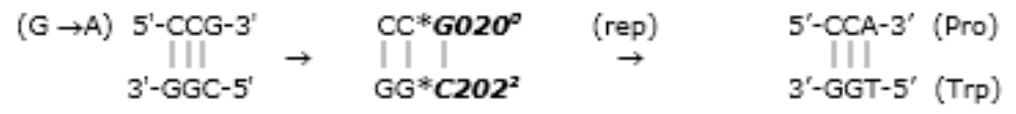

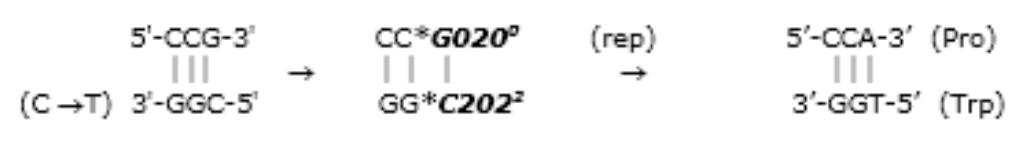

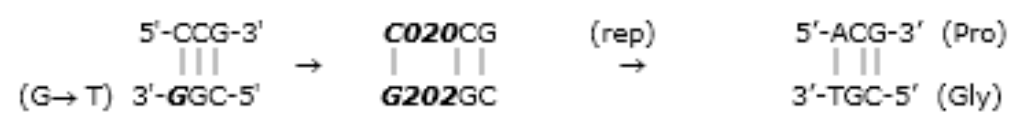

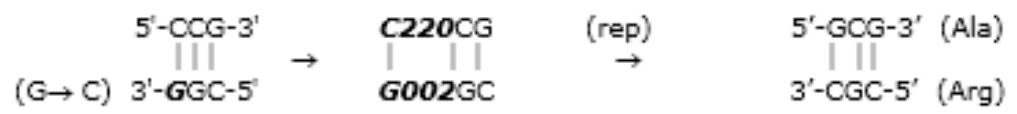

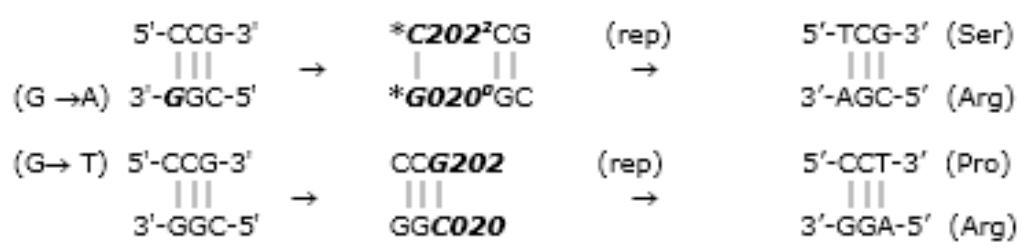

Figure 6a. Entangled proton qubits populating CCG repeats

(Figure 6. Base substitution pathways generated by EPR arrangements, keto-amino $\rightarrow$ enol-imine, introducing entangled proton qubit states in duplex triplet repeats of (a) CCG/GGC and (b) CAG/GTC. The particular substitutions are in parentheses, e.g., $(C \rightarrow T)$, adjacent to the reactive $5^{\prime}$ or $3^{\prime}$ strand of the triplet duplex. The initial product is selected by the proton qubit "trapped" in a DNA groove [73-74], $\delta \mathrm{t}<<$ $10^{-13} \mathrm{~s}$, which identifies the participating eigenstate of the $\boldsymbol{G}^{\prime}-\boldsymbol{C}^{\prime}$ or ${ }^{*} \boldsymbol{G}-{ }^{*} \boldsymbol{C}$ superposition within the triplet duplex. Subsequent transcription (trans) and/or replication (rep) of enol and imine proton qubit isomers within STRs yield altered triplet codes, where pathways for generating initiation codons and stop codons 
W. Grant Cooper; Evolution Via EPR-Entanglement Algorithm. Journal of Biomedical Engineering and Medical Imaging, Volume 4, No 2, April (2017), pp 43-95

are indicated. The CUG initiation codon can be derived from keto-amino CAG/GTC as indicated. Notation specifying particular proton qubit states is that of Fig. 2.)

DNA versus limited expansions (copy no. < 1000) in male haploid DNA [28-29] are attributed to an increased energy density of duplex DNA [112-113] in $37{ }^{\circ} \mathrm{C}$ oocyte genomes $[2,110]$.

At transcription before replication, $\delta \mathrm{t} \leq 10^{-13} \mathrm{~s}$, accumulated entangled proton qubit states, $5^{\prime}-\mathrm{C}^{*} \mathbf{C} \mathbf{2 0 2}^{2} \mathrm{G}$ 3' and 5'-CG'202G-3', would express additional "new" initiation codons, 5'-CUG-3'. In the "neighborhood" of initiation regions for DNA replication [104], such additional reinitiating signals could cause an addition of more triplet repeats, which would be manifested as (CCG) $n_{n}$ expansion [28-29,78,109]. Thus at transcription just before fertilization of an oocyte [110], accumulated entangled proton qubit superposition states - C*C202 ${ }^{2} \mathrm{G}$ and CG'202G (Fig. 6a) - could be transcribed to yield CUG, which can also specify reinitiating of DNA synthesis, thereby causing massive $(C C G)_{n}$ expansion in oocyte DNA $[28,109]$. Figure $6 a$ also shows that twelve of the twenty-one (57\%) ts that code for an amino acid would result in amino acid substitution.

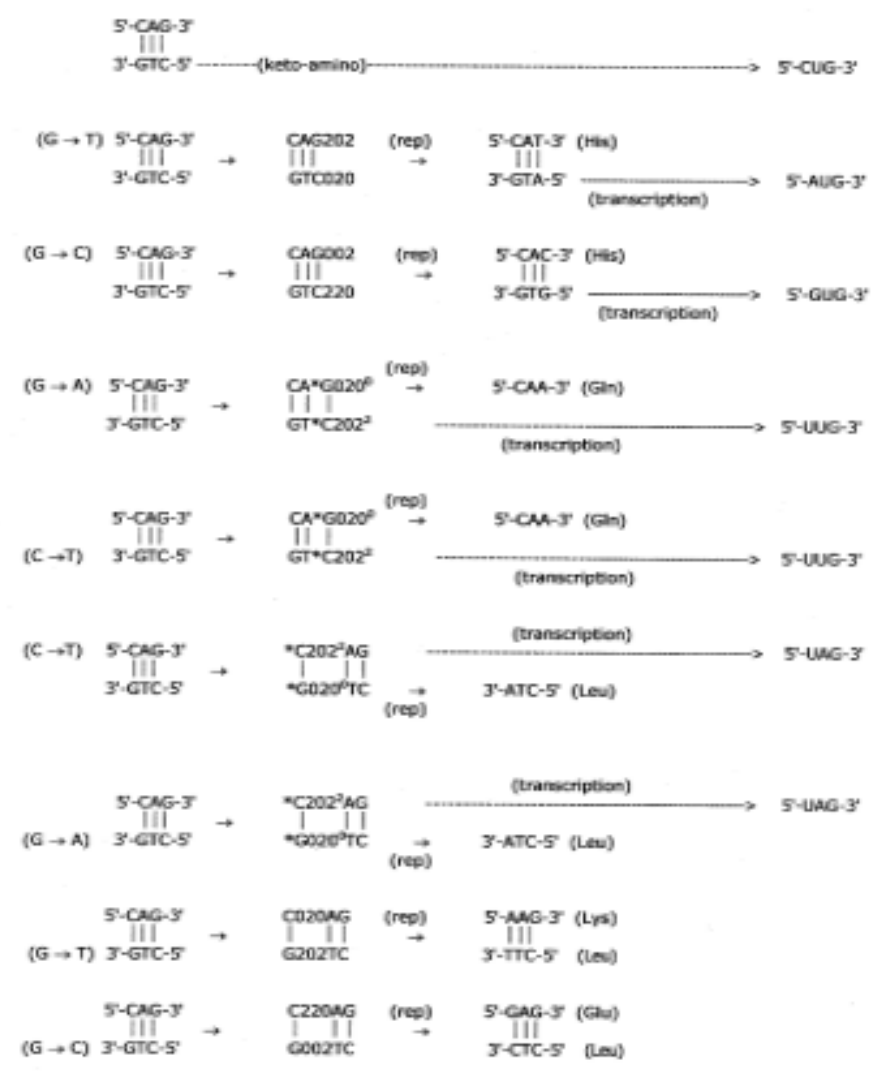

Figure $6 \mathrm{~b}$. Entangled proton qubits populating CAG repeats

Unstable CAG repeats are responsible for several neurological diseases [33], including Huntington's disease [1-2,6,37,114-115]. Figure 6b illustrates that EPR-generated entangled proton qubits accumulated in CAG-repeats could express initiation codons, AUG and GUG, which would require replication, whereas the two UUG codons could be expressed by quantum transcription prior to replication. Similarly, the two UAG stop codons would be expressed by quantum transcription before replication. Observation that CAG 
expansion is more efficient in sperm [116-118] implies that replication-dependent pathways would be primarily responsible for CAG expansion in haploid human DNA. Expression of UAG codons would be responsible for nonsense mutations, and thus, contractions are observed in CAG tracts from sperm [118]. This combination of expansion and contraction modes would govern instability exhibited by CAG repeats [1-2,6,118]. According to Fig. 6b, 5'-CAG-3' and 5'-CTG-3' are complementary components on opposite strands of a duplex repeat, 5'-CAG/GTC-5'. However, ordinary keto-amino 5'-CTG-3' could generate the CUG initiation codon by transcription without ts intervention. Amino acid substitutions would be introduced in four of the ten ts that code for amino acids. In particular, Gln would be replaced by Glu, Lys and His (twice).

and Contraction and contraction evolutionary dynamics and CT repeats1)arequantum entanglement algorithm, 1-2,6,29,entangled proton qubits and A-Tby which entangled proton qubits populate 77 for ing entangled proton qubit,entangled proton qubits7entangled proton qubit [1-2] (Data on A-T rich triplets are not displayed).

entangled proton qubit70 0220 2entangled the terminationcode - 5'-UAG-3',7-isentangled proton qubit7 (in both rat and human172,20,22. 
W. Grant Cooper; Evolution Via EPR-Entanglement Algorithm. Journal of Biomedical Engineering and Medical Imaging, Volume 4, No 2, April (2017), pp 43-95

$$
\underset{||}{\text { 5'-CAC-3' }}
$$

-(keto-amino)

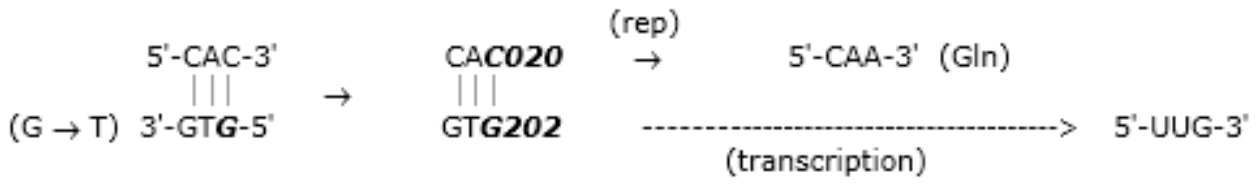

$$
\begin{aligned}
& (\mathrm{C} \rightarrow \mathrm{T}) \quad 5^{\prime}-\mathrm{CAC}-3^{\prime} \quad \mathrm{CA}^{*} \mathrm{C2}^{202} \text { (rep) 5'-CAT-3' (His) } \\
& \text { 3'-GTG-5' } \text { GT*GO20 }^{\circ} \rightarrow \text { 3'-GTA-5' }
\end{aligned}
$$
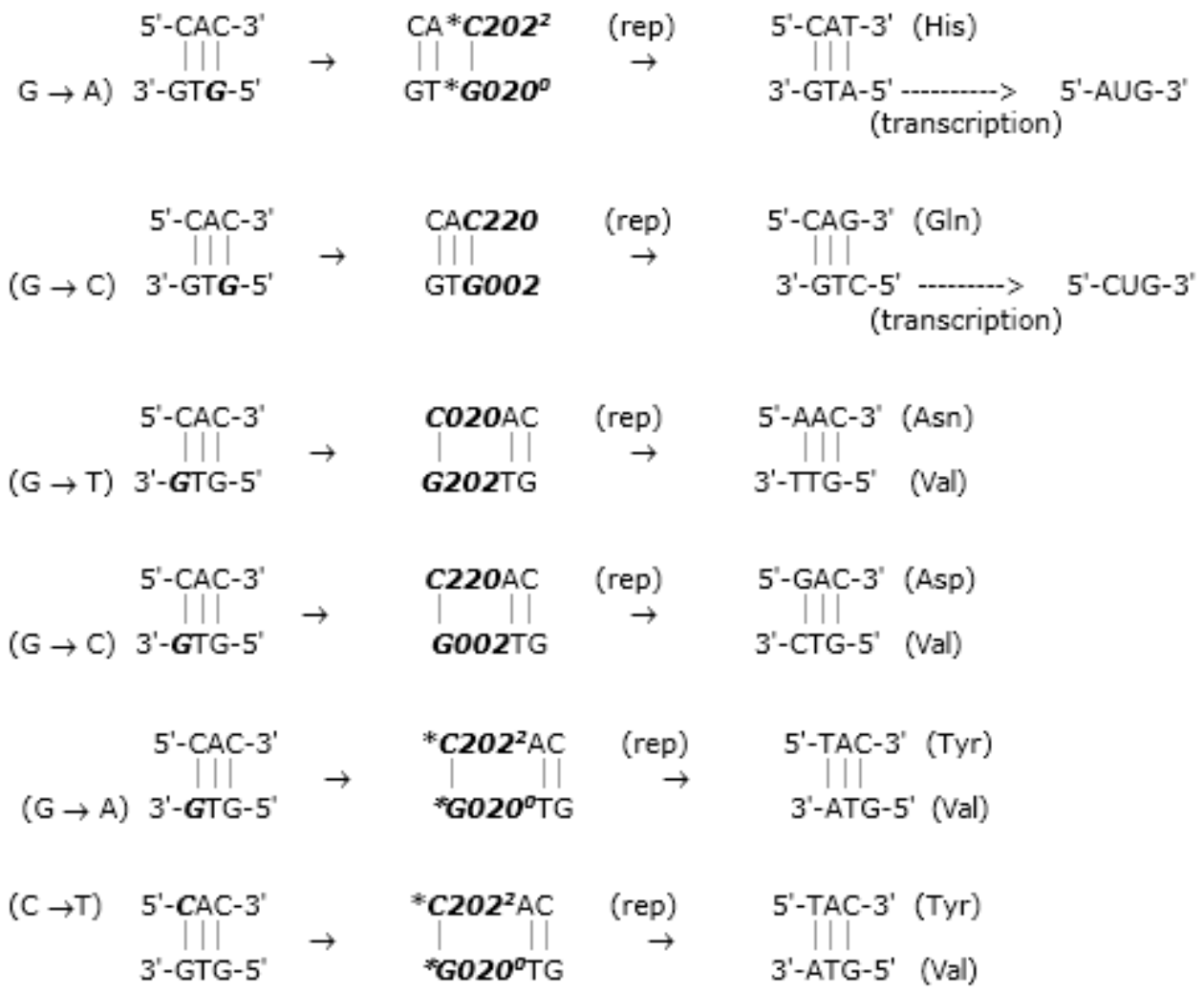

Figure 7a. Entangled proton qubits populating CAC/GTG triplets 


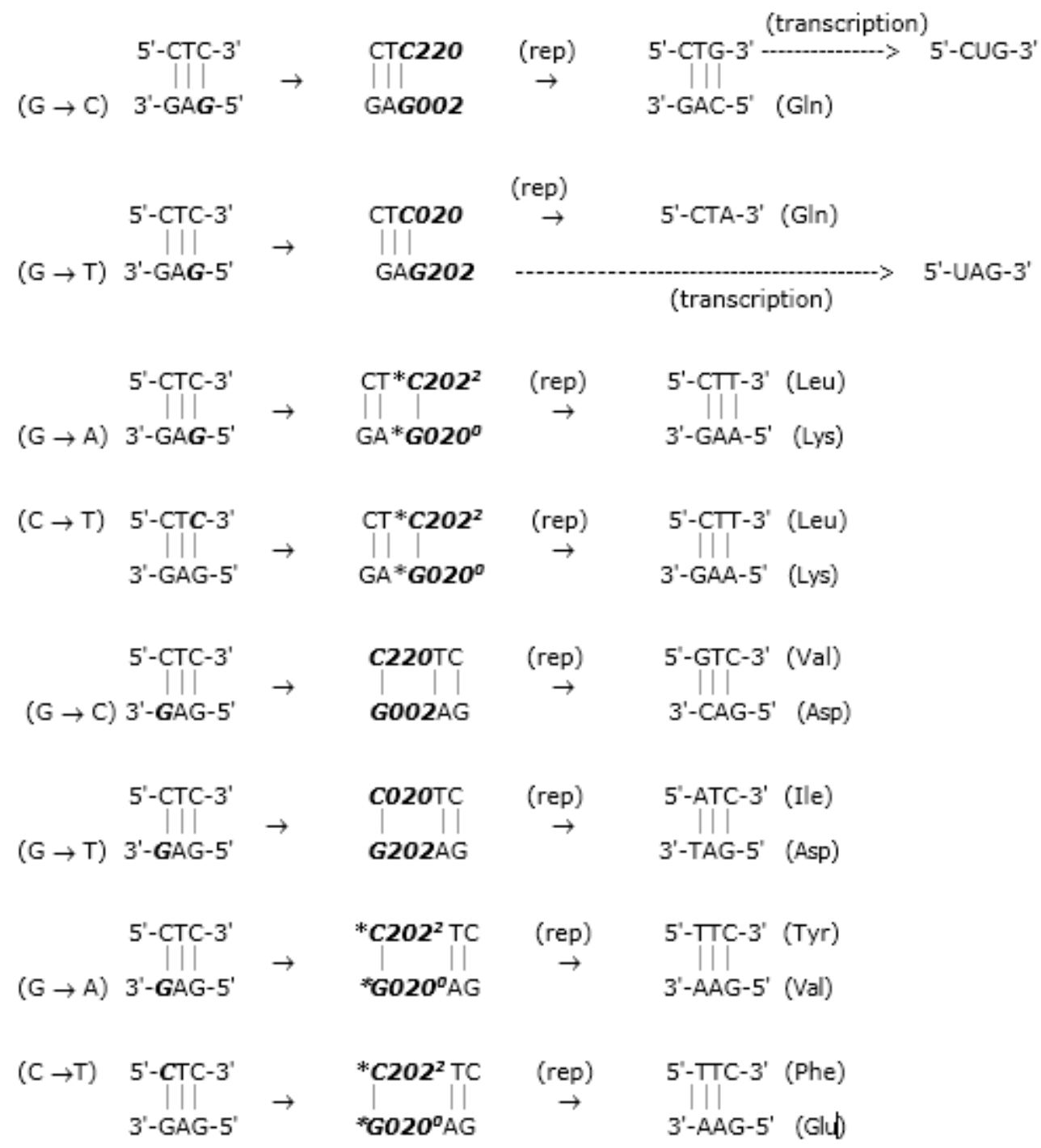

Figure 7b. Entangled proton qubits populating CTC/GAG triplets.

(Figure 7. Pathways for generating entangled proton qubit states in STRs of (a) CAC/GTG and (b) CTC/GAG where decohered isomers are replicated to yield time-dependent substitutions, ts. The particular substitutions are in parentheses adjacent to the reactive $5^{\prime}$ or $3^{\prime}$ strand of the duplex triplet. The initial product identifies the "selected" eigenstate of the $\boldsymbol{G}^{\prime}-\boldsymbol{C}^{\prime}$ or ${ }^{*} \boldsymbol{G}-{ }^{*} \boldsymbol{C}$ quantum superposition within the STR, using Fig. 2 notation. Subsequent transcription (trans) and/or replication (rep) of STRs yield the resulting triplet codes. Pathways for entangled proton qubit states to generate initiation and stop codons are indicated. Introduction of the $5^{\prime}$-GUG-3' initiation codon by the keto-amino CAC/GTG duplex is shown. Notation specifying states of entangled proton qubits is given in Fig. 2.)

20221ancient 4444example,4. 
W. Grant Cooper; Evolution Via EPR-Entanglement Algorithm. Journal of Biomedical Engineering and Medical Imaging, Volume 4, No 2, April (2017), pp 43-95

Table 4a. Eleven microsatellites exhibiting "expansion" in ancient rat genome

\begin{tabular}{|c|c|c|c|c|c|c|}
\hline 1 & 2 & 3 & 4 & 5 & 6 & 7 \\
\hline Motif & $\begin{array}{l}\text { Relative A } \\
\text { (Number of }\end{array}$ & $\begin{array}{l}\text { Abundance } \\
\text { f STRs) }\end{array}$ & $\# \mathrm{H} / \# \mathrm{R}$ & $\begin{array}{l}\text { \& Increase } \\
\text { Relative } \\
\text { Abundance }\end{array}$ & $\begin{array}{l}\text { \& Pathways } \\
\text { INITIATION } \\
\text { Codons }\end{array}$ & $\begin{array}{l}\text { \& Pathways } \\
\text { STOP } \\
\text { Codons }\end{array}$ \\
\hline & Rat & Human & & & & \\
\hline $\mathrm{ACGC}$ & 7 & 1 & 0.14 & $600 \%$ & $33 \%$ & 8.3 옹 \\
\hline $\mathrm{AGGG}$ & 5 & 1 & 0.20 & $400 \%$ & $16.6 \%$ & $8.3 \%$ \\
\hline $\mathrm{CAGA}$ & 6 & 2 & 0.33 & $200 \%$ & $50 \%$ & 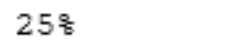 \\
\hline CT & 56 & 19 & 0.34 & $195 \%$ & $12.5 \%$ & $12.5 \%$ \\
\hline (b) $\mathrm{ACC}$ & 6 & 3 & 0.50 & $100 \%$ & $32 \%$ & 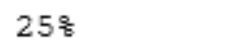 \\
\hline $\mathrm{CA}$ & 133 & 73 & 0.55 & 82 & $50 \%$ & 0 올 \\
\hline AAGG & 13 & 8 & 0.62 & 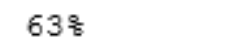 & 25 & 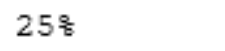 \\
\hline $\mathrm{CAG}$ & 11 & 9 & 0.82 & 22 옴 & $50 \%$ & 25 \\
\hline AATG & 6 & 3 & 0.50 & 100 온 & $0 \&$ & 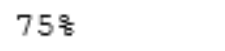 \\
\hline $\mathrm{ACAT}$ & 4 & 3 & 0.75 & 33 & $0 \%$ & 50 옹 \\
\hline AATT & 3 & 1 & 0.33 & $200 \%$ & $0 \%$ & $0 \%$ \\
\hline
\end{tabular}
a) keto-amino CAGA/GTCT allows CUG expression
b) ACC includes CAC STRs; Averaged values in column 6 \& 7
c) keto-amino AATG allows AUG and UAA expression
d) keto-amino ACAT/TGTA allows AUG expression
e) keto-amino AATT allows UAA expression 
Table 4b. Eleven microsatellites exhibiting "contraction" in ancient rat genome

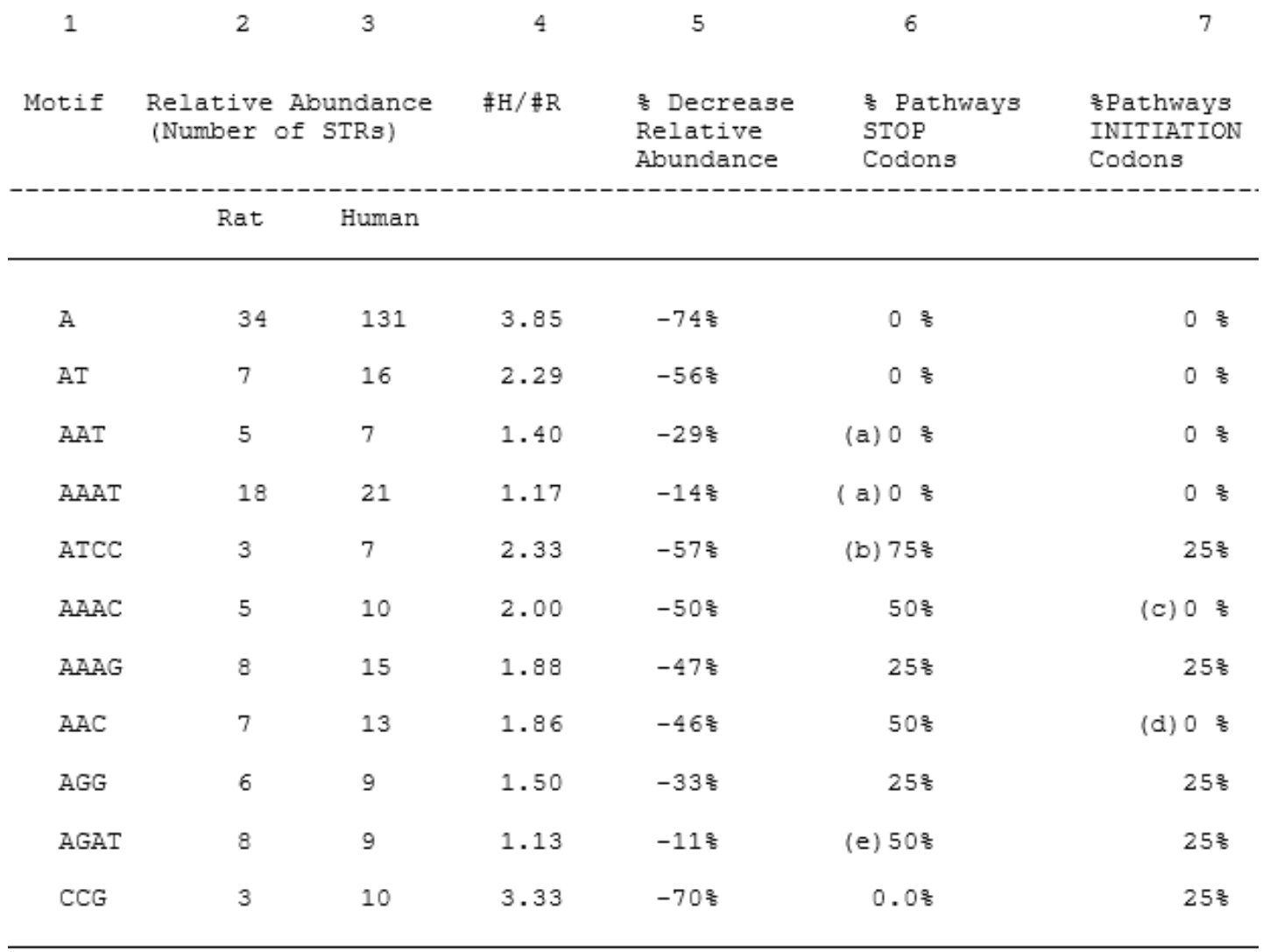

(a) keto-amino AAT and AAAT allow UAA expression

(b) keto-amino ATCC/TAGG allows UAG expression

(c) keto-amino AAAC/TTTG allows UUG expression

(d) keto-amino AAC/TTG allows UUG expression

(e) keto-amino AGAT/TCTA allows UAG expression

quantum entanglement algorithm 1-2,6-7,29 measurements of entangled proton qubitterminationmeasurements of entangled proton qubit [6,9-11]107EPR-generated entangled proton qubitinterval for ng entangled proton qubittherefore entanglement-enabled Consequently,4 [107] EPRgeneratedentangled proton qubit - that yield the "expansion" mode -Similarly,4entangled proton qubitentangled proton qubit "ancient"entangled proton qubit4 results8entangled qubit4 results9CTC; 78entangled proton qubit4 Table 4a,,entangled proton qubit464 enzyme quantum reader measurements of entangled proton qubits generating quantum transcription expressions of1-3,911Consequently,8848eses48 Additionally,

(Figure 8. Pathways for entangled proton qubit introduction of dynamic mutations expressed as initiation codons and stop codons in STRs of (a) ACGC, (b) AGGG, (c) CAGA, (d) AAGG, (e) AATG and (f) ACAT. Pathways for expressing initiation and stop codons by keto-amino STRs are indicated.). 
W. Grant Cooper; Evolution Via EPR-Entanglement Algorithm. Journal of Biomedical Engineering and Medical Imaging, Volume 4, No 2, April (2017), pp 43-95

(Insert Fig. 8a,b,c,d,e,f below)

\begin{tabular}{|c|c|c|c|c|c|c|c|}
\hline$(G \rightarrow A)$ & $\begin{array}{l}\text { ACGC } \\
|||| \\
\text { TGCG }\end{array}$ & $\rightarrow$ & $\mid \mathrm{T}^{*} \mathrm{~A}^{*} \mathrm{C} 2022^{\circ} \mathrm{GC}$ & $\begin{array}{c}\text { (rep) } \\
\rightarrow\end{array}$ & $\begin{array}{l}\text { ATGC } \\
|\|| \mid \\
\text { TACG }\end{array}$ & (transcription) & $5^{\prime}-A \cup G-3^{\prime}$ \\
\hline$(\mathrm{C} \rightarrow \mathrm{T})$ & $\begin{array}{l}\text { ACGC } \\
\|\|_{T C G}\end{array}$ & $\rightarrow$ & $\begin{array}{c}\mathrm{A}^{*} \mathrm{C2O2}^{2} \mathrm{GC} \\
\mid \mathrm{T}^{*} \mathrm{GO2O} 0^{\circ} \mathrm{CG}\end{array}$ & $\begin{array}{l}\text { (rep) } \\
\rightarrow\end{array}$ & $\begin{array}{l}\text { ATGC } \\
\|\| \mid \\
\text { TACG }\end{array}$ & $\overrightarrow{\text { (transcription) }}$ & $5^{\prime}-A \cup G-3^{\prime}$ \\
\hline$(G \rightarrow A)$ & $\begin{array}{l}\text { ACGC } \\
\|\| \| \\
\text { TGCG }\end{array}$ & $\rightarrow$ & $\begin{array}{l}\mathrm{AC}^{*} \mathrm{GO2O}^{\circ} \mathrm{C} \\
|||||| \mid \\
\mathrm{TG}^{*} \mathrm{C}^{2} 02^{2} \mathrm{G}\end{array}$ & $\begin{array}{l}\text { (rep) } \\
\quad \rightarrow\end{array}$ & $\begin{array}{l}\text { ACAC } \\
|\|||| \\
\text { TGTG }\end{array}$ & (transcription) & $5^{\prime}$-GUG-3' \\
\hline$(\mathrm{C} \rightarrow \mathrm{T})$ & $\begin{array}{l}\text { ACGC } \\
|||| \\
\text { TGCG }\end{array}$ & $\rightarrow$ & $\begin{array}{l}\mathrm{AC}^{*} \mathrm{GO20}^{\circ} \mathrm{C} \\
|| \\
\mathrm{TG}^{*} \mathrm{C2O2}^{2} \mathrm{G}\end{array}$ & $\begin{array}{l}\text { (rep) } \\
\rightarrow\end{array}$ & $\begin{array}{l}\text { ACAC } \\
\mid \| G T G\end{array}$ & (transcription) & $5^{\prime}-$ GUG-3' \\
\hline$(\mathrm{G} \rightarrow \mathrm{T})$ & $\begin{array}{l}\text { ACGC } \\
|||| \mid \\
\text { TGCG }\end{array}$ & $\rightarrow$ & $\begin{array}{l}\text { ACG202C } \\
|| \mid \\
\text { TGCO20G }\end{array}$ & $\begin{array}{l}\text { (rep) } \\
\rightarrow\end{array}$ & $\begin{array}{l}\text { ACTC } \\
\|\| \mid \\
\text { TGAG }\end{array}$ & (transcription) & $=5^{\prime}-$ UGA-3' \\
\hline$(G \rightarrow T)$ & $\begin{array}{l}\text { ACGC } \\
|||| \mid \\
\text { TGCG }\end{array}$ & $\rightarrow$ & $\begin{array}{l}\mathrm{AC020GC} \\
|||| \mid \\
\mathrm{TG202CG}\end{array}$ & $\begin{array}{c}\text { (rep) } \\
\rightarrow\end{array}$ & $\begin{array}{l}\text { AAGC } \\
|||| \\
\text { TTCG }\end{array}$ & & \\
\hline$(G \rightarrow C)$ & $\begin{array}{l}\text { ACGC } \\
|||| \\
\text { TGCG }\end{array}$ & $\rightarrow$ & $\begin{array}{l}\mathrm{AC220GC} \\
|||| \mid \\
\mathrm{TG002CG}\end{array}$ & $\underset{\rightarrow}{(\text { rep) }}$ & $\begin{array}{l}\text { AGGC } \\
\|\| \mid \\
\text { TCCG }\end{array}$ & & \\
\hline$(G \rightarrow C)$ & $\begin{array}{l}\text { ACGC } \\
\|\| \| \\
\text { TGCG }\end{array}$ & $\rightarrow$ & $\begin{array}{l}\text { ACG002C } \\
|||| \mid \\
\text { TGC220G }\end{array}$ & $\begin{array}{c}(\text { rep }) \\
\rightarrow\end{array}$ & $\begin{array}{l}\mathrm{ACCC} \\
|\|| \mid \\
\mathrm{TGGG}\end{array}$ & & \\
\hline$(G \rightarrow T)$ & $\begin{array}{l}\text { ACGC } \\
|||| \\
\text { TGCG }\end{array}$ & $\rightarrow$ & $\begin{array}{l}\mathrm{ACGCO20} \\
|||| \\
\text { TGCG202 }\end{array}$ & $\begin{array}{c}\text { (rep) } \\
\rightarrow\end{array}$ & $\begin{array}{l}\text { ACGA } \\
\|\||| \\
\text { TGCT }\end{array}$ & & \\
\hline$(G \rightarrow C)$ & $\begin{array}{l}\text { ACGC } \\
|||| \\
\text { TGCG }\end{array}$ & $\rightarrow$ & $\begin{array}{l}\text { ACGC220 } \\
\||| \mid \\
\text { TGCG002 }\end{array}$ & $\begin{array}{c}\text { (rep) } \\
\rightarrow\end{array}$ & $\begin{array}{l}\text { ACGG } \\
\|\| \mid \\
\text { TGCC }\end{array}$ & & \\
\hline$(G \rightarrow A)$ & $\begin{array}{l}\text { ACGC } \\
|||| \\
\text { TGCG }\end{array}$ & $\rightarrow$ & $\begin{array}{l}\left.\mathrm{ACG}^{*} \mathrm{C}_{\mid}\right|_{\mathrm{TGC}}{ }^{2} \\
\mathrm{TGO}^{*} \mathrm{GO2O}^{\circ}\end{array}$ & $\begin{array}{l}\text { (rep) } \\
\rightarrow\end{array}$ & $\begin{array}{l}\text { ACGT } \\
\|\||| \\
\text { TGCA }\end{array}$ & & \\
\hline$(\mathrm{C} \rightarrow \mathrm{T})$ & $\begin{array}{l}\text { ACGC } \\
|||| \\
\text { TGCG }\end{array}$ & $\rightarrow$ & $\begin{array}{l}\mathrm{ACG}{ }^{*} \mathrm{C}^{\mathrm{AO2} 2} \mathrm{2}^{2} \\
||||\end{array}$ & $\begin{array}{l}\text { (rep) } \\
\rightarrow\end{array}$ & $\begin{array}{l}\text { ACGT } \\
\|\| \mid \\
\text { TGCA }\end{array}$ & & \\
\hline
\end{tabular}

Figure 8a. ACGC: EPR-generated dynamic mutations 


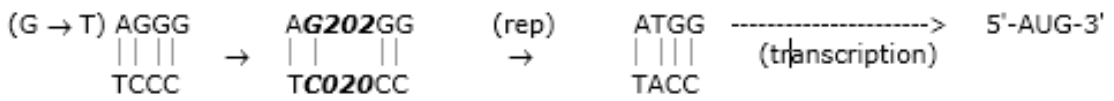

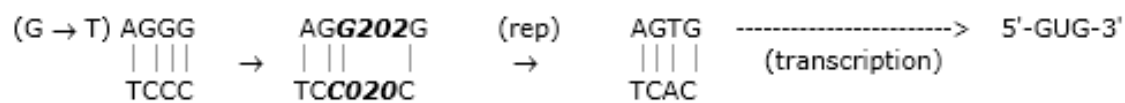

$$
\begin{aligned}
& \text { AGTG } \\
& |||| \text { TCAC (transcription) }
\end{aligned}
$$

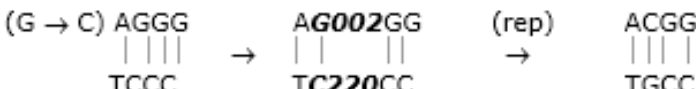

$$
\begin{aligned}
& (\mathrm{G} \rightarrow \mathrm{A}) \text { AGGG } \quad \mathrm{A}^{*} \mathrm{GO2O}^{\circ} \mathrm{GG}(\mathrm{rep}) \quad \text { AAGG }
\end{aligned}
$$

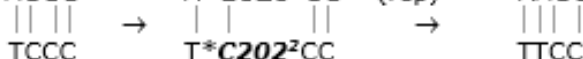

$$
\begin{aligned}
& \text { AGGG A*G020 }{ }^{\circ} \mathrm{GG} \text { (rep) AAGG } \\
& (\mathrm{C} \rightarrow \mathrm{T}) \underset{\mathrm{TCCC}}{\|} \underset{\mathrm{T}^{*} \mathbf{C 2 O 2}^{\mathbf{C}} \mathrm{CC}}{\rightarrow} \stackrel{\|}{\| C C} \\
& (\mathrm{G} \rightarrow \mathrm{C}) \text { AGGG } \quad \text { AGG002G } \quad \text { (rep) AGCG } \\
& \underset{\mathrm{TCCC}}{\mid \|} \underset{\mathrm{TCC220C}}{\|} \rightarrow \underset{\mathrm{TCGC}}{\|} \\
& (\mathrm{G} \rightarrow \mathrm{A}) \text { AGGG } \rightarrow \stackrel{\mathrm{AG}^{*} \mathrm{GO2O}^{\circ} \mathrm{G}}{(\mathrm{rep})} \underset{\rightarrow}{\mathrm{AGAG}} \\
& \mathrm{TCCC} \rightarrow \mathrm{TC}^{*} \mathrm{C2O2}^{2} \mathrm{C} \rightarrow \text { TCTC } \\
& \text { AGGG } \quad \mathrm{AG}^{*} \mathrm{GO}^{\circ} \mathrm{O}^{\circ} \mathrm{G} \text { (rep) AGAG }
\end{aligned}
$$

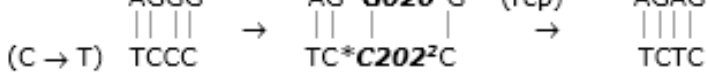

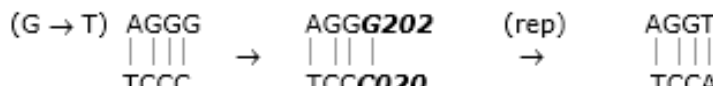

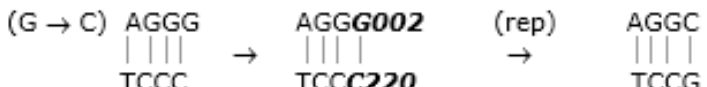

$$
\begin{aligned}
& (\mathrm{G} \rightarrow \mathrm{A}) \text { AGGG AGG*G020 (rep) AGGA }
\end{aligned}
$$

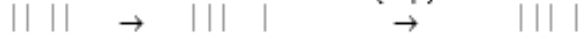

$$
\begin{aligned}
& \mathrm{TCCC} \rightarrow \mathrm{TCC}^{*} \mathrm{C2O2}^{2} \rightarrow \text { TCCT }
\end{aligned}
$$

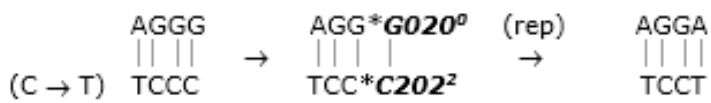

Figure 8b. AGGG: EPR-generated dynamic mutations 
W. Grant Cooper; Evolution Via EPR-Entanglement Algorithm. Journal of Biomedical Engineering and Medical Imaging, Volume 4, No 2, April (2017), pp 43-95

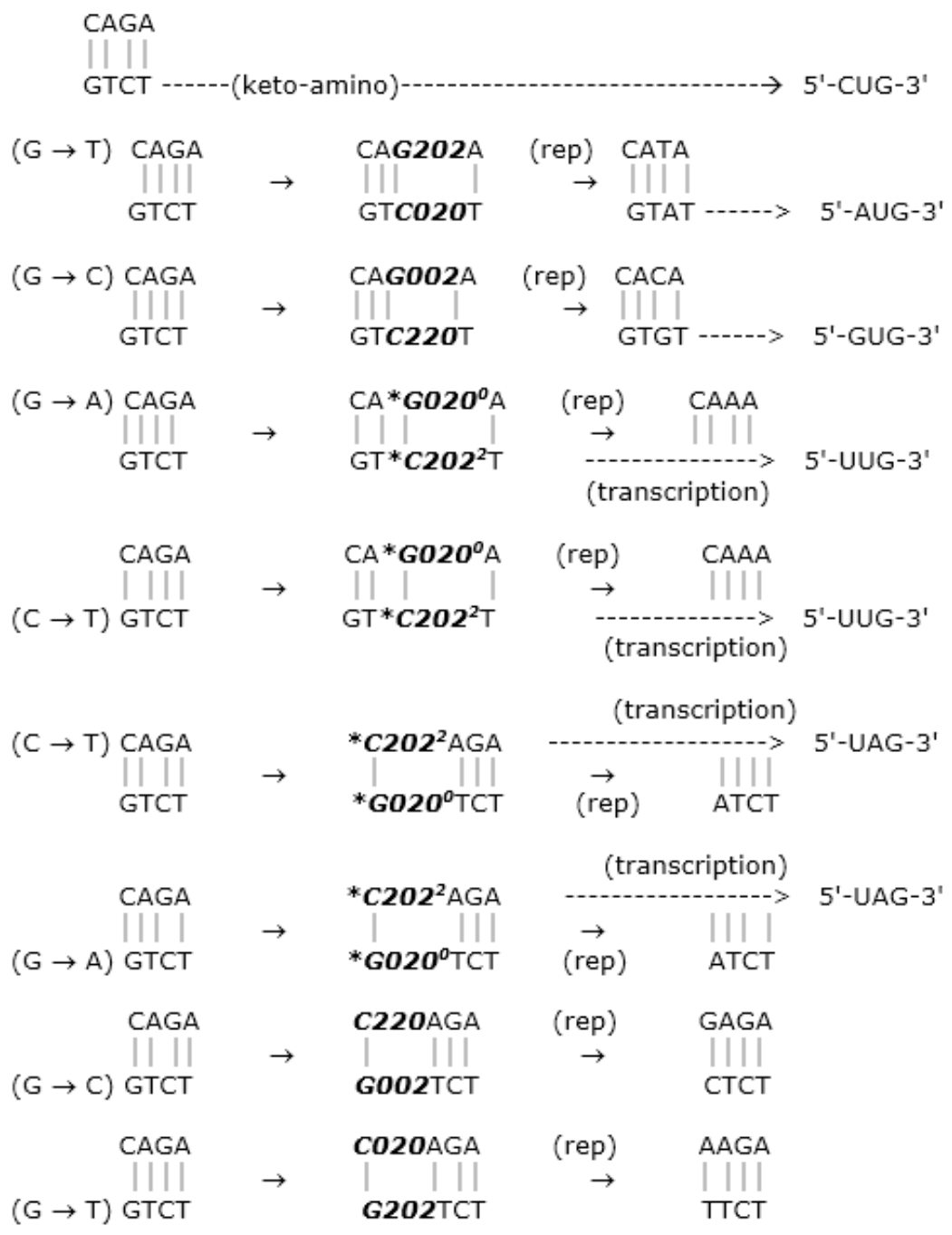

Figure 8c. CAGA: EPR-generated dynamic mutations 


$$
\begin{aligned}
& (G \rightarrow C) \text { AAGG AAGOO2G (rep) AACG } \\
& \|\| \rightarrow\|M \quad \rightarrow \quad\||| \text { (transcription) } \\
& \text { TTCC TTCO20C TTGC }---\cdot m \text { 5'-UUG-3' }
\end{aligned}
$$

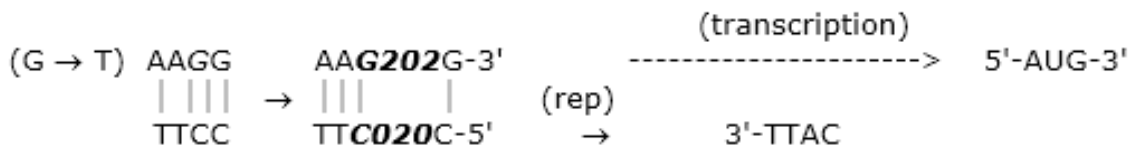

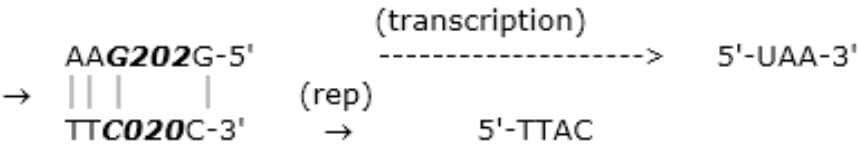

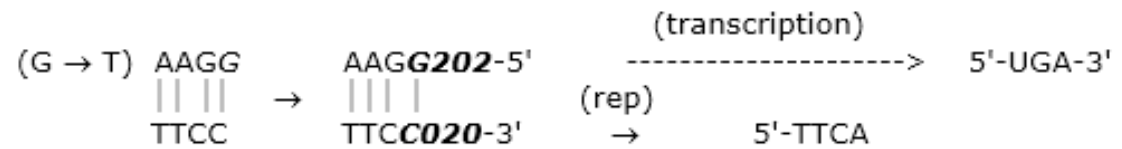

$$
\begin{aligned}
& \left(G \rightarrow \text { A) AAGG } \quad \text { AA* }^{*} \mathbf{G O 2 O}^{\circ} \mathrm{G}\right. \text { (rep) AAAG } \\
& \underset{T T C C}{||||} \rightarrow \underset{T{ }^{*} \mathbf{C 2 O 2}^{2} \mathrm{C}}{\rightarrow} \stackrel{\text { TTC }}{\rightarrow}
\end{aligned}
$$

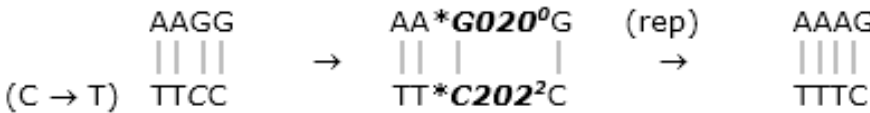

$$
\begin{aligned}
& (G \rightarrow C) \text { AAGG } \quad \text { AAGGOO2 } \quad(\text { rep }) \quad \text { AAGC } \\
& \underset{\text { TTCC }}{\|\|} \rightarrow \underset{\text { TTCCO2O }}{\|||} \stackrel{\text { TTCG }}{\rightarrow} \\
& (\mathrm{G} \rightarrow \mathrm{A}) \text { AAGG } \quad \text { AAG* }^{*} \mathrm{GO2O}^{\circ} \text { (rep) AAGA } \\
& \underset{T T C C}{\|||} \underset{T T C * \mathbf{C 2 O 2}^{2}}{\|} \rightarrow \text { TTCT }
\end{aligned}
$$

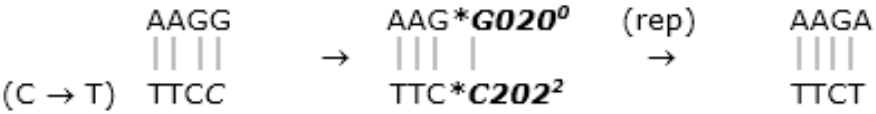

Figure 8d. AAGG: EPR-generated dynamic mutations 
W. Grant Cooper; Evolution Via EPR-Entanglement Algorithm. Journal of Biomedical Engineering and Medical Imaging, Volume 4, No 2, April (2017), pp 43-95

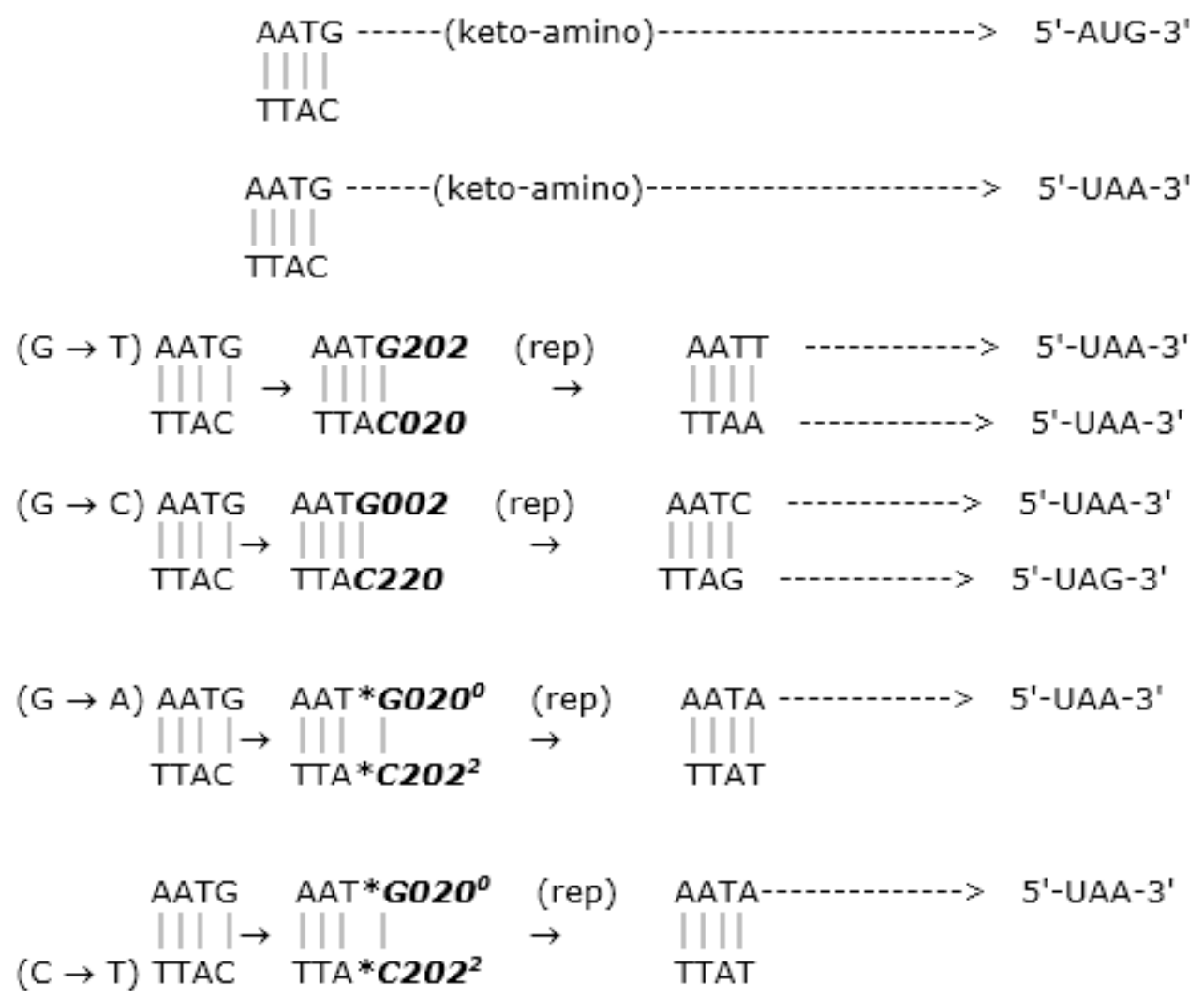

488 ,entangled proton qubit4

$44-44$

Figure 8e. AATG: EPR-generated dynamic mutations 


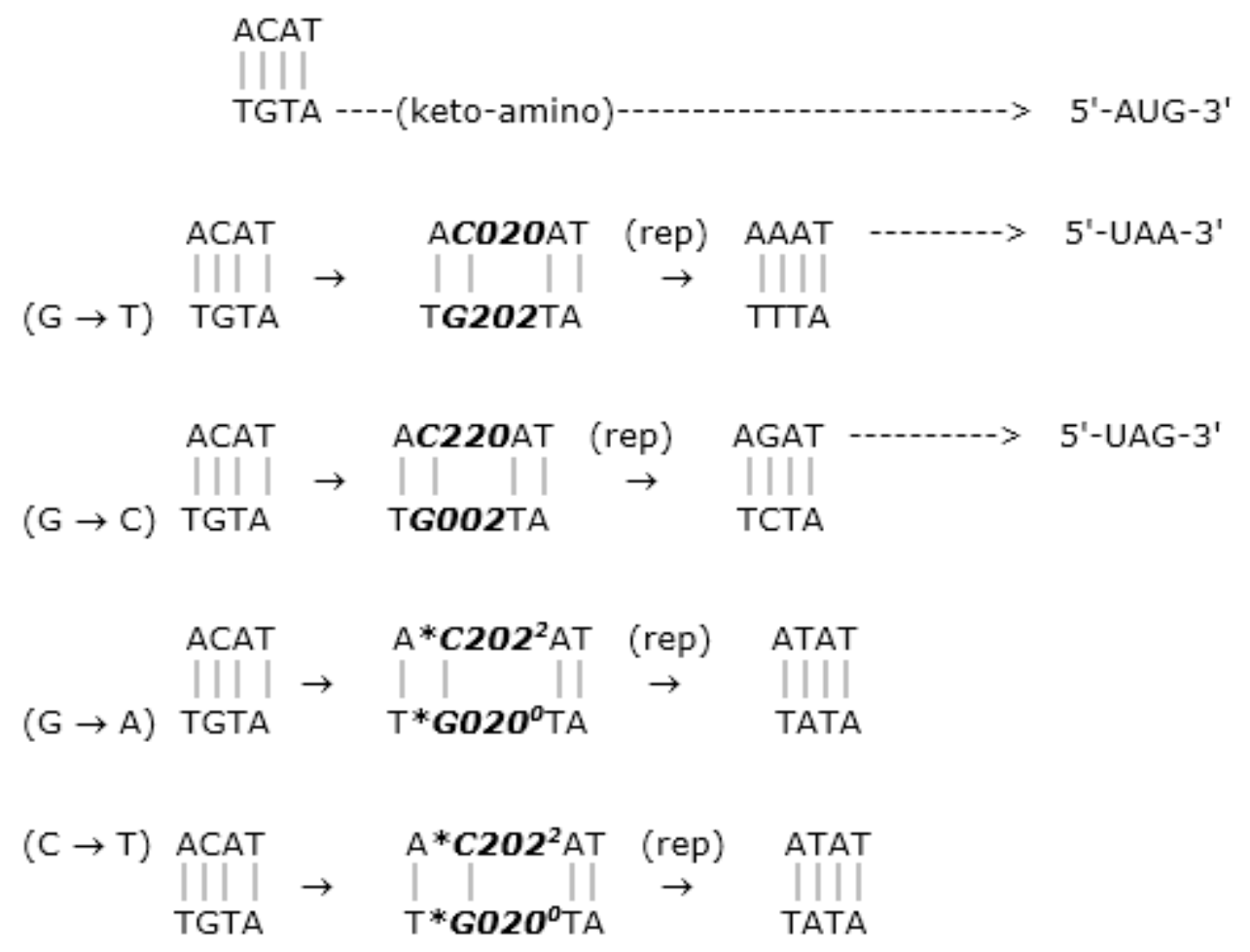

Figure 8f. ACAT: EPR-generated dynamic mutations

82028 entangled proton qubit 46-entangled proton qubit 9and AGG9fentangled proton qubit474entangled proton qubit9entangled proton qubit s99EPR-generated entangled proton qubit49EPR entangled proton qubit494--4entangled proton qubit9

(Figure 9. Pathways for entangled proton qubit introduction of dynamic mutations expressed as initiation codons and stop codons in STRs of (a) ATCC, (b) AAAC, (c) AAAG, (d) AAC, (e) AGG and (f) AGAT. Pathways for expressing initiation and stop codons by keto-amino STRs are indicated.) 
W. Grant Cooper; Evolution Via EPR-Entanglement Algorithm. Journal of Biomedical Engineering and Medical Imaging, Volume 4, No 2, April (2017), pp 43-95

ATCC

|| $\mid$

TAGG ------(keto-amino)------------------> 5'-UAG-3'

(rep)

$\begin{array}{lll}\text { ATCC } & \text { ATCO20C } \rightarrow \quad \text { ATAC }\end{array}$

$\|\quad\| \quad|| \quad$ (transcription)

$(\mathrm{G} \rightarrow \mathrm{T}) \quad$ TAGG TAG202G $\quad-\cdot-\cdot-\cdot-\cdot-\cdot-\cdot>\quad 5 '-A U G-3 '$

ATCC ATC220C (rep) ATGC ---> 5'-AUG-3'

|| $\rightarrow \quad \| \mid$ | $\rightarrow|| \mid$

$(\mathrm{G} \rightarrow \mathrm{C}) \quad$ TAGG $\quad$ TAG002G TACG

ATCC $\quad \mathrm{AT}^{*} \mathbf{C 2 0 2}^{\mathbf{2}} \mathrm{C}$ (rep) ATTC

||$|\rightarrow||| \rightarrow|| \mid$

$(\mathrm{G} \rightarrow \mathrm{A}) \quad \mathrm{TAGC} \quad \mathrm{TA} * \mathbf{G 0 2 0 ^ { \circ } \mathrm { G }} \quad$ TAAG $--->\quad 5^{\prime}-\mathrm{UAA}-3^{\prime}$

$(\mathrm{C} \rightarrow \mathrm{T}) \quad$ ATCC $\quad \mathrm{AT}^{*} \boldsymbol{C 2 0 2}^{2} \mathrm{C}$ (rep) ATTC

|| $\rightarrow \quad|||\quad \rightarrow \quad||| \mid$

TAGC TA*G020 ${ }^{\circ} \mathrm{G} \quad$ TAAG $--->5^{\prime}-U A A-3 '$

(rep)

ATCC $\quad 3 '-A T C C 020 \rightarrow$ ATCA

||$|\rightarrow \quad|||$

$(\mathrm{G} \rightarrow \mathrm{T}) \quad$ TAGG $\quad$ 5'-TAGG202

5'-UAG-3'

(transcription)

(rep)

5'-ATCCO20 $\rightarrow \quad$ ATCA

$\rightarrow \quad|| \mid$

3'-TAGG202

5'-UAG-3'

(transcription) 


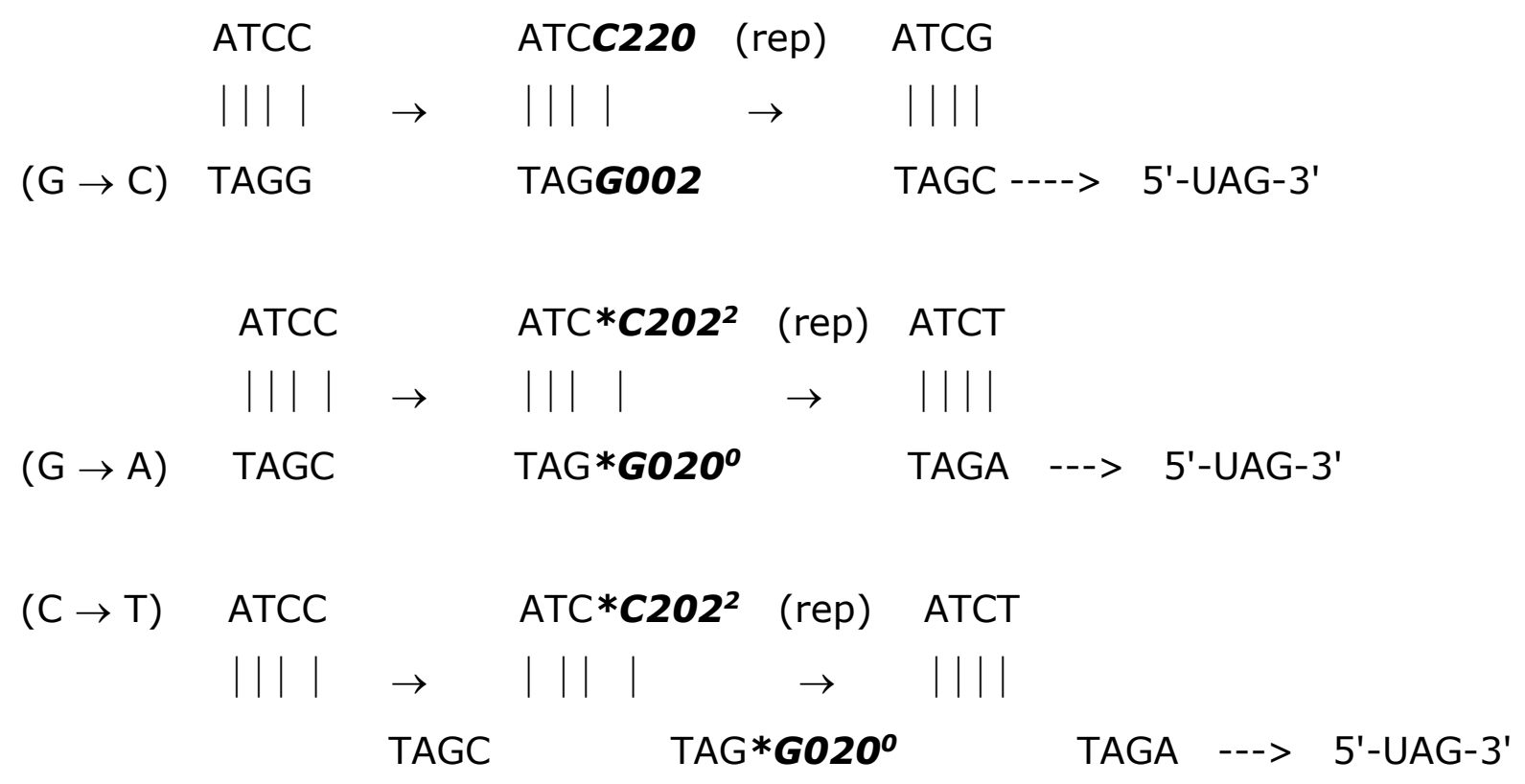

Figure 9a. ATCC: EPR-generated dynamic mutations 
W. Grant Cooper; Evolution Via EPR-Entanglement Algorithm. Journal of Biomedical Engineering and Medical Imaging, Volume 4, No 2, April (2017), pp 43-95

\section{AAAC}

|| ||

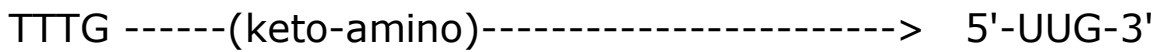

(transcription)

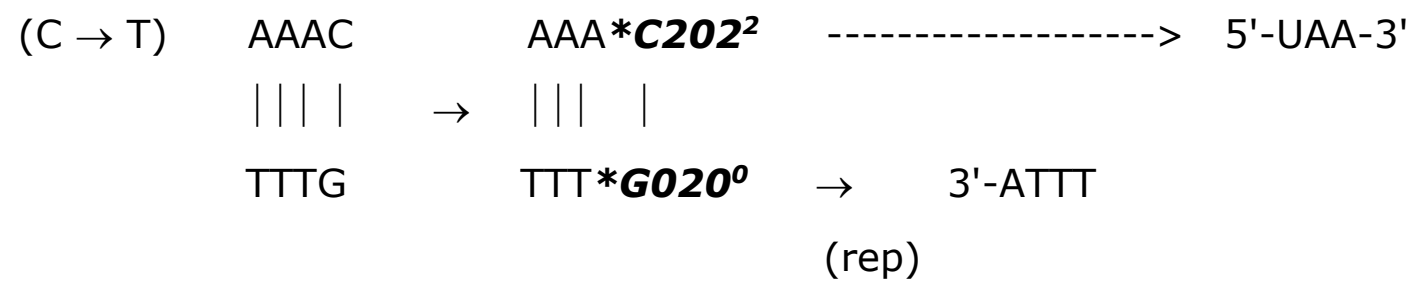

(transcription)

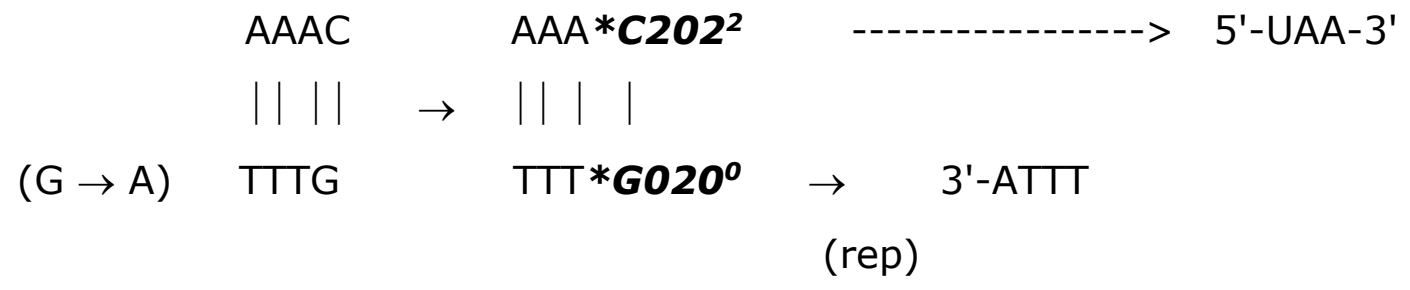

\begin{tabular}{|c|c|c|c|c|c|}
\hline & AAAC & & AAACO20 & (rep) & AAAA \\
\hline & \|\| & $\rightarrow$ & $|1|$ & $\rightarrow$ & || \\
\hline$(\mathrm{G} \rightarrow \mathrm{T})$ & TTTG & & ТТТ 202 & & TाTा \\
\hline & AAAC & & AAAC220 & (rep) & AAAG \\
\hline & $|1|$ & $\rightarrow$ & 111 & $\rightarrow$ & \\
\hline$(G \rightarrow C)$ & TTTG & & ТТТG002 & & TाT \\
\hline
\end{tabular}

Figure 9b. AAAC: EPR-generated dynamic mutations

99 greater decrease --4DNA (16 generated by the quantum entanglement algorithm operating on (CCG) repeats $1-2,294444$

\section{Discussion and Conclusion}

Confidence in entanglement-enabled bio-molecular information processing [1-4,12] is provided by the fact that multiple lines of experimental observation - prokaryote T4 phage systems [2,9-11] and human gene systems [1-2,4,6-7,29] - converge with the EPR-generated, time-dependent molecular evolution model for STRs herein analysed and discussed [1-4]. Quantum uncertainty limits, $\Delta x \Delta p_{x} \geq \hbar / 2$, operate on 
metastable amino $\left(-\mathrm{NH}_{2}\right)$ hydrogen bonded protons, which introduces a probability of EPR-generated entangled proton qubit superposition states, keto-amino -(entanglement) $\rightarrow$ enol-imine, in STRs. Grover's [12] quantum processor measurements of dynamic entangled proton qubit states predict a timedependent creation of initiation codons, stop codons and deletions [1-3,6-7,29], and consequently, provide a mechanism $(\boldsymbol{a})$ for stochastic random genetic drift, $\boldsymbol{t} \boldsymbol{s}+\boldsymbol{t} \boldsymbol{d}[9-11,19,49]$, and $(\boldsymbol{b})$ for expansion and contraction of STRs $[1-3,6-7,29,78,104]$. In addition to quantum chemical analyses identifying two internally consistent, "ordered sets" of expanding and contracting STRs from the list of twenty-two (Table 1) most abundant STRs common to human and rat, Table $4 a$ provides insight into evolutionary "expansion" processes. For example, a rationale is presented for $(C A)_{n}$ repeats to be longer and more numerous than $(\mathrm{CT})_{\mathrm{m}}$ repeats in both human and rat. General agreement between model expectation and relative abundance of STRs in rat and human genomes implies that evolutionary processes of expansion and/or contraction can be simulated in terms of EPR-generated entangled proton qubit superposition states populating $\boldsymbol{G}^{\prime}-\boldsymbol{C}^{\prime},{ }^{*} \boldsymbol{G}-{ }^{*} \boldsymbol{C} \&{ }^{*} \boldsymbol{A}-{ }^{*} \boldsymbol{T}$ sites in STRs, which are subsequently processed by the quantum entanglement algorithm [1-4,12]. An apparent consequence of these evolutionary processes includes triplet repeat genetic instabilities [1-2,28-29,33-34,78,109] and phenotypic expression - as a function of time (age) - of associated triplet repeat human diseases [1-2,6-7,33,108,114-115].

1s 44144 However, obtained metastable hydrogen bonding amino $\left(-\mathrm{NH}_{2}\right)$ protons encountering quantum uncertainty limits, $\Delta x \Delta p_{x} \geq \hbar / 2$, which generates probabilities of EPR arrangements, where position momentum quantum entanglement is introduced between separating enol and imine protons [1-3,1316]. Reduced energy product protons are each shared between two indistinguishable sets of electron lone-pairs belonging to enol oxygen and imine nitrogen on opposite strands, and consequently, participate in entangled quantum oscillation at $\sim 10^{13} \mathrm{~s}^{-1}$ between near symmetric energy wells in decoherence-free subspaces [63-65] until "measured by" [1-3,6] a "Grover's-type" enzyme quantum processor [7-12]. V(Tables 4a-b) 44 entanglement algorithma rational for (Table 4a) (Table 4b) Results of squantum entanglement algorithmic processing of entangled proton qubits are 69. quantum entanglement algorithm predictions [1-3,6,29] 


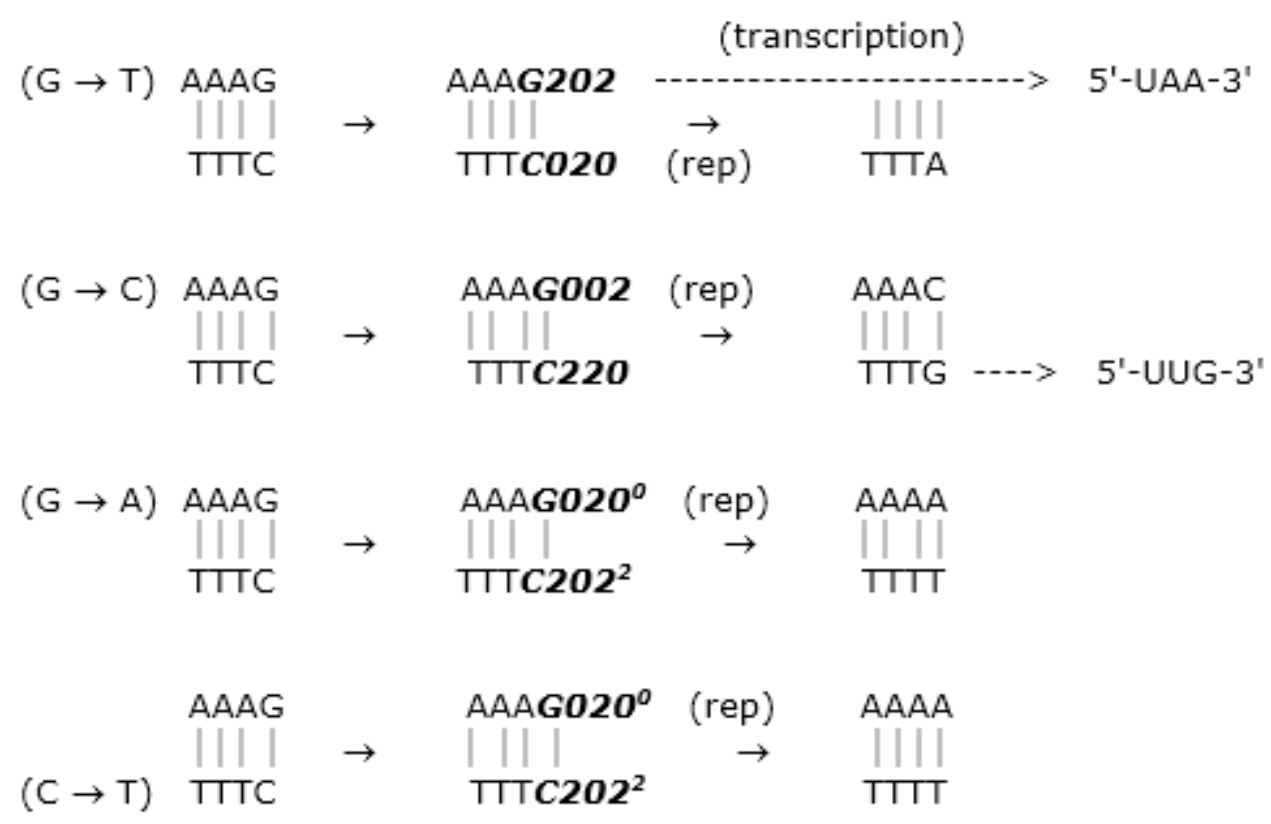

Figure 9 c. AAAG: EPR-generated dynamic mutations

[20] the 22 most abundant rat and human [20-23]quantum entanglement algorithm [1-4,6-12,29]which Quantum entanglement algorithm analysesof $(\boldsymbol{a})$ evolving distributions of human-rodent STRs $[2,20],(\boldsymbol{b})$ ancient T4 phage DNA [2,9-11,80-82], and (c) human gene systems [1-2,4, 6-8] are consistent with the hypothesis ancestral - ribozyme systems initially acquired and "processed" EPR-generated entangled proton qubits [3,12], cellular (LUCA) [67-70,103] Subsequent selection of enzyme - proton entanglement processing of entangled proton qubits was an "early" adaptive mutation [1-3,7,119] that allowed development and growth of an increasingly complex, evolving genomic system. The fact that EPRgenerated $\boldsymbol{t}$ s and $\boldsymbol{t} \boldsymbol{d}$ can introduce and eliminate initiation codons - UUG, CUG, AUG, GUG - and termination codons - UAA, UGA, UAG - implies resultant "dynamic" mutations [1-3,6-7,28-29,78,108109] played significant roles in physical genomic growth, which has provided the classical duplex molecular matrix on which the quantum entanglement algorithm operates on dynamic, EPR-generated entangled proton qubits [1-4,6-11]. Availability of enzyme - proton entanglement processing [12] of entangled proton qubits [1-4] allowed growth in genomic mass, i.e., additional base pair units via "expansion" [1-3,6,29,78], which enhanced the probability introducing additional EPR-generated entangled proton qubits. Most of life's evolutionary stages - e.g., precellular, cellular, eukaryogenesis, etc. $[3,67-70,103,120]$ - have successfully emerged under conditions of continuous accumulations of entangled proton qubits deciphered by quantum processing 


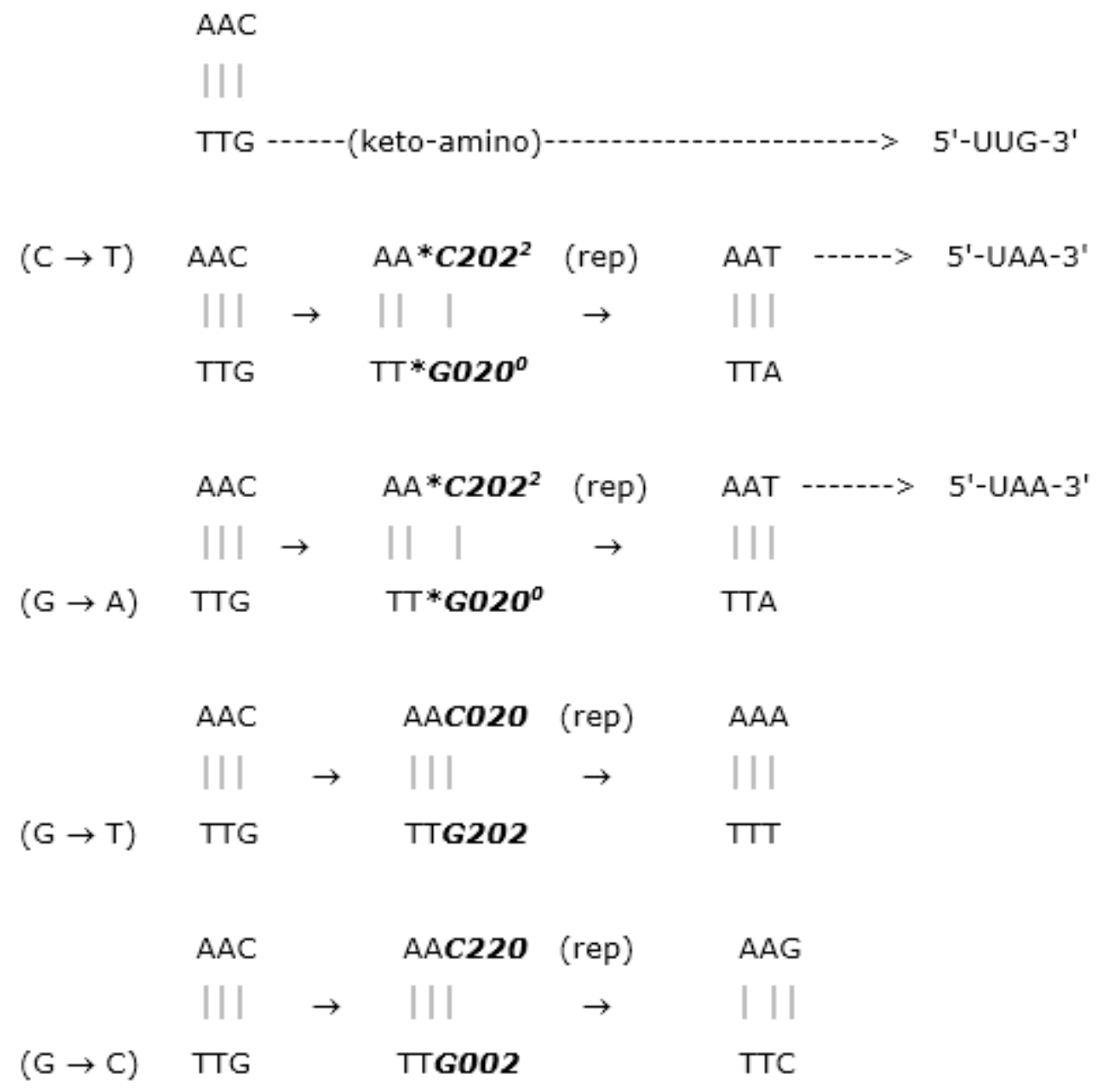

Figure 9d. ACC: EPR-generated dynamic mutations

information enzymes (QPIE) to yield $\boldsymbol{t}$ s and $\boldsymbol{t} \boldsymbol{d}$, but several evolutionary consequences have not been "accurately" recognized [1-12,29]. According to the evolution scenario outlined here, over the past 3.5 or so billion y [1-3,67-70], enzyme quantum-reader 


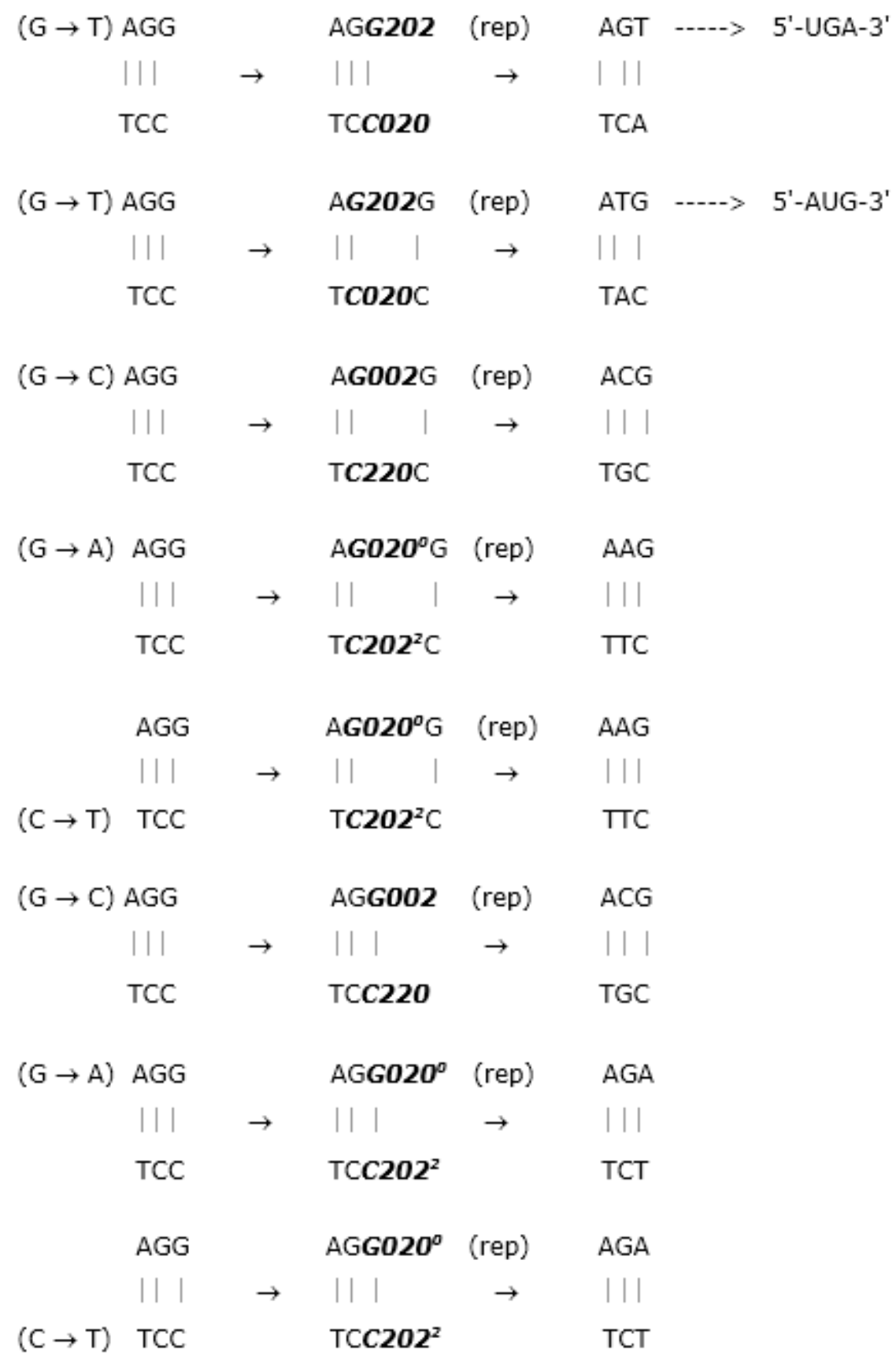

Figure 9e. AGG: EPR-generated dynamic mutations

processing [12] of EPR-generated entangled proton qubits have provided an entanglement resource for quantum dynamical genomic growth and evolution, from relatively primitive pre-LUCA systems [3,67-70] into the more complex and biologically diverse, modern mammalian genomic system [1-4,6$7,29,103,120]$. This growth and development in operational biological complexity is thus a consequence of Darwinian selection operating on enzyme - proton entanglement processes, driven by availability of EPR-generated entangled proton qubits at the microphysical, entangled proton qubit genomic level. According to this and other reports [1-4], the smallest enzymatically measurable unit of quantum- 
entanglement genetic information is an "entangled pair" of EPR-generated proton qubits, occupying decoherence-free subspaces [1-3,63-65], and subsequently measured by Grover's [12] quantum processor. Consequently, a nucleotide is not the smallest "basic" unit of genetic information measured by enzyme processors responsible for molecular genomic evolution [1-3].

In duplex DNA of human genomes, unstable repeats [1-2,28-29,33,108-109] exhibit expansions and contractions via dynamic mutations $[6-7,29,78]$, where $(C A G)_{n}$ sequences $(n>36)$ can exhibit expansions $\geq 10$ (CAG) repeats in 20 y [1,114-115]. This observation implies the hypothesis that susceptible ancestral genomes implemented dynamic mutation expansions as consequences of particular ts $+\boldsymbol{t} \boldsymbol{d}$ [1-4,6-11]. A "net" triplet repeat dynamic mutation expansion rate of 13 repeats, e.g., (CAG) $)_{13}=39$ bp, per 20 y for 3.5 billion y would generate a genome of $\sim 6.8 \times 10^{9} \mathrm{bp}$, which is "ballpark" compatible with bp content of the Homo sapiens' genome. According to the present assessment, evolutionary genomic growth was, and is, a consequence of the quantum entanglement algorithm introducing, and eliminating, initiation codons - UUG, CUG, AUG, GUG - and stop codons, UAA, UAG \& UGA. This hypothesis is consistent with the fact that overall microsatellite content in a genome correlates with genome size of the prokaryote or eukaryote organism [23]. Selected "expansion" sequences were exploited as conserved genes, e.g. [121125], whereas "other" expansion sequences have been relegated to "unspecified" conserved noncoding genomic space (CNGS) [126-127]. An "accurate" understanding of quantum entanglement algorithm evolution of STRs appears to provide new and useful insight into unusual behavior exhibited by Huntington's disease $(C A G)_{n}$ repeats $[1-2,6,114]$ and other unstable triplet repeat diseases $[28-29,33,108$ 109]. Specifically, quantum entanglement algorithm analyses of STR evolution data - Tables $1,4 a, 4 b-$ support the hypothesis that the $\sim 2$ to 

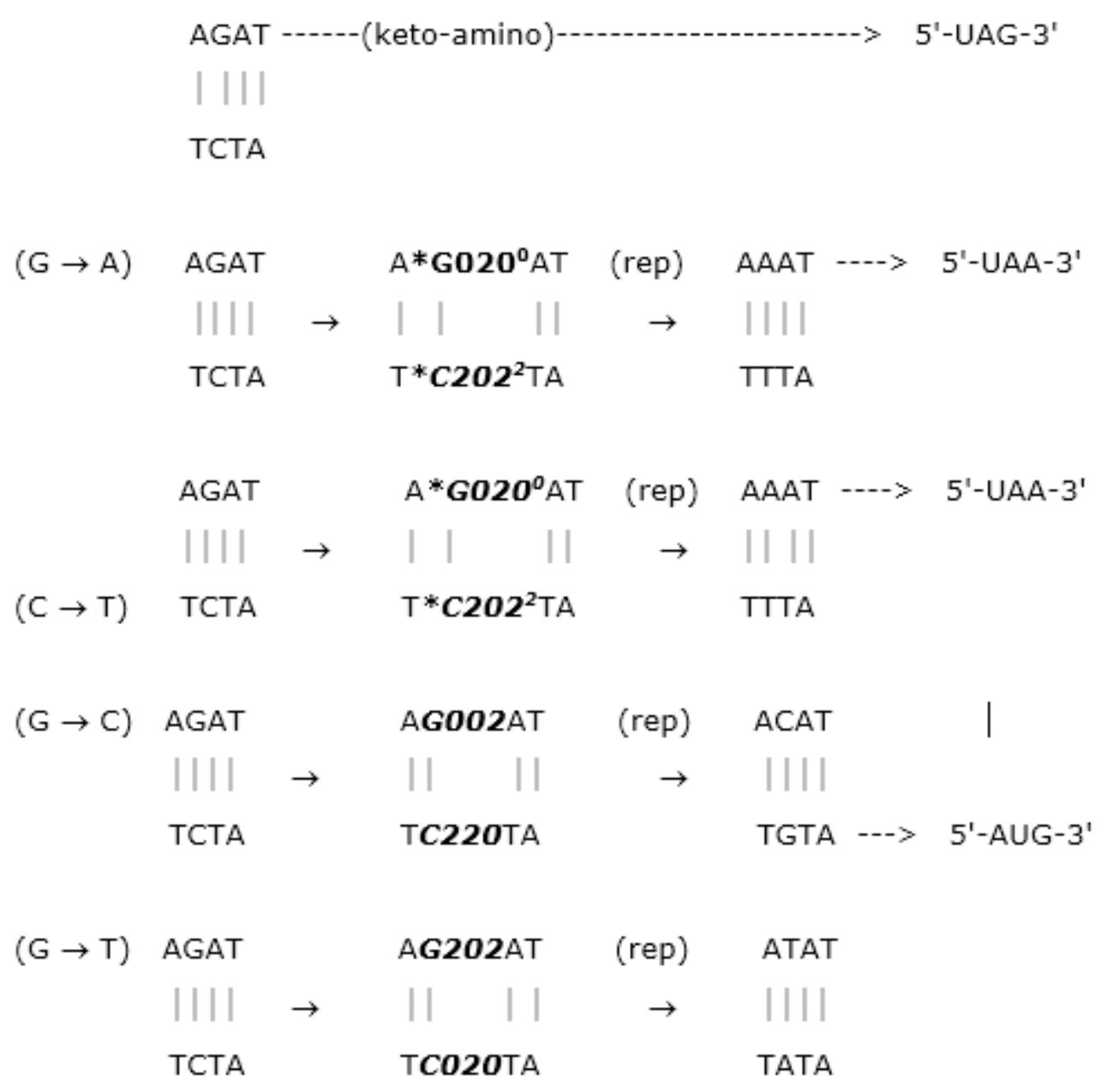

Figure 9f. AGAT: EPR-generated dynamic mutations

$\sim 12$ yr. delay in expression of Huntington's disease by an inherited long, e.g., (CAG) 70 repeat [114], is due to the necessity of Grover's [12] transcriptase processors measuring available entangled proton qubits occupying a "threshold limit" [115] of the inherited (CAG) $n(n \geq 70)$ repeat [1].

The quantum entanglement algorithm [1-4] generates a probabilistic yield of entangled proton qubit states, which would manifest an irregular 'tick rate', as observed [9-11,49,128]. Also, the expression of mutagenic codes, i.e., expansions and/or contractions, would introduce additional variations into microsatellite molecular clock data. Thus the quantum entanglement algorithm seems to provide a plausible mechanism - at the microscopic entangled proton qubit information level [1-4] - for generating differences in substitution [6-7,9], $t \boldsymbol{s}$, and deletion [10-11], $\boldsymbol{t} \boldsymbol{d}$, rates $[49,128]$. By incorporating these measurable features [1-11] into models where mathematical variables and operations represent quantifiable biological reality, one could aim for a reduction in parameters and an improved accuracy in models that analyze genetic distance between species [1-3,5,49,128]. Based on the high level of qualitative agreement between model prediction and observation of STRs [20], this report concludes that microsatellite evolution $[23,114]$ can be simulated in terms of EPR-generated, entangled proton qubit states - measured by Grover's [12] quantum processors - which introduces $\boldsymbol{t} \boldsymbol{s}+\boldsymbol{t} \boldsymbol{d}$ that can cause expansions and contractions [1-2,6-7,29]. Agreement between model predictions and observed STR 
evolution of the 22 "most abundant" microsatellites (Table 1) implies significant elements of correctness regarding EPR-generated, molecular mechanisms responsible for genome and microsatellite evolution, and thus, warrants further theoretical and experimental investigations. This study also implies availability of a time evolution quantum entanglement algorithmic approach for investigating properties of past and future microsatellites.

\section{ABBREVIATIONS}

CNGS = conserved noncoding genomic spaces

$E P R=$ Einstein-Podolsky-Rosen, "EPR", refers to ref. 13, entitled, "Can Quantum-Mechanical Description of Physical Reality be Considered Complete?" (Phy. Rev. 1935, 47, 777-780.) This paper presented a central debate over the interpretation of quantum theory where the concept of "entanglement" was introduced. The paper considers two quantum systems that physically interact - before separation - such that both their spatial coordinates are linked in a certain direction, and also their linear momenta are linked in the same direction. As a result of this "entanglement", either position or momentum determination for one separated system would fix (respectively) the position or the momentum of the other separated system, instantaneously.

LUCA = last universal cellular ancestor

QPIE = quantum processing information enzymes

SNP = single nucleotide polymorphism

STRs $=$ short tandem repeats

ts $=$ entanglement originated time-dependent substitution

$\boldsymbol{t} \boldsymbol{d}=$ entanglement originated time-dependent deletion

$5 \mathrm{HMC}=5$-hydroxymethylcytosine

\section{ACKNOWLEDGEMENT}

Useful conservations with Roy Frieden on quantum coherence and decoherence of enzyme - proton entanglements in biological systems are gratefully appreciated. I thank Jeremy Greiner for assistance in creating several figures used in discussions of evolutionary distributions of human-rodent microsatellites. The author states conflicts of interest do not exist. 
W. Grant Cooper; Evolution Via EPR-Entanglement Algorithm. Journal of Biomedical Engineering and Medical Imaging, Volume 4, No 2, April (2017), pp 43-95

\section{REFERENCES}

[1] Cooper WG (2016) Molecular dynamics responsible for observable Huntington's disease (CAG) repeat evolution. Ann. Neurodegener Dis 1(2) 1009.

[2] Cooper WG (2017) Origin of Life Insight: Reactive Transitions from Anthropic Causality to Biological Evolution. OMICS Group eBooks (in press), www.esciencecentral.org/ebooks.

[3] Cooper WG (2016) Quantum information processing model explains "early" and "recent" genome repair mechanisms, Res. \& Rev.: J. Pure \& Applied Phys.

[4] Cooper WG (2018) Consequences of EPR-generated entangled proton qubits origin of life model. (Submitted to Adv. Quantum Chem. 77, November 2016).

[5] Hwang DG, Green P (2004) Bayesian Markov chain Monte Carlo sequence analysis reveals varying neutral substitution patterns in mammalian evolution. Proc. Natl. Acad. Sci. USA, 101:13994-14001.

[6] Cooper WG (2012) Coherent states as consequences of keto-amino $\rightarrow$ enol-imine hydrogen bond arrangements driven by quantum uncertainty limits on amino DNA protons. Int. J. Quantum Chem. 112:2301-2323.

[7] Cooper WG (2011) The molecular clock in terms of quantum information processing of coherent states, entanglement and replication of evolutionarily selected decohered isomers. Interdiscip. Sci. 3: 91-109.

[8] Cooper, WG (2011) Accuracy in biological information technology involves enzymatic quantum processing and entanglement of decohered isomers. Information 2:166-194.

[9] Cooper, W.G., (1994) T4 phage evolution data in terms of a time dependent Topal-Fresco mechanism, Biochem. Genet. 32, 383395.

[10] Cooper WG (2009) Evidence for transcriptase quantum processing implies entanglement and decoherence of superposition proton states. BioSystems 97:73-89.

[11] Cooper WG (2009) Necessity of quantum coherence to account for the spectrum of time-dependent mutations exhibited by bacteriophage T4. Biochem. Genet 47: 392-410.

[12] Grover LK (1996) A fast quantum mechanical algorithm for database search. In: Proc. 28th Annual ACM Symposium on the Theory of Computing, ACM, Philadelphia, p.212; Phys Rev. Lett. 1997, 79: 325.

[13] Einstein A, Podolsky B, Rosen N (1935) Can Quantum Mechanical Description of Physical Reality be Considered Complete? Phy. Rev. 47: 777-780.

[14] Schrödinger E, Born M (1935) Discussion of probability relations between separated systems. Mathematical Proceedings of the Cambridge Philosophical Society 31: 555-563. 
[15] Schrödinger E (1936) Discussion of Probability Relations between Separated Systems, Proceedings of the Cambridge Philosophical Society 31:555-563; 1936, 32:446-451.

[16] Schrödinger E, Dirac PAM (1936) Probability relations between separated systems. Mathematical Proceedings of the Cambridge Philosophical Society 32: 446-452.

[17] Bell JS (1964) On the Einstein-Podolsky-Rosen Paradox. Physics 1: 195-200.

[18] Bell JS (1993) Speakable and Unspeakable in Quantum Mechanics. Cambridge University Press.

[19] Whitney KD, Garland T, Jr (2010) Did genetic drift drive increases in genome complexity? PLoS Genet. 26: e1001080.

[20] Beckmann, J.S., Weber, J.L. (1992) Survey of human and rat microsatellites, Genomics 12, 627631.

[21] Weber J.L., Wong C. (1993). Mutation of human short tandem repeats. Hum. Mol. Genet. 2 (8): 11231128. doi:10.1093/hmg/2.8.1123.

[22] Love JM, Knight AM, McAleer MA, Todd JA (1990) Towards construction of a high-resolution map of the mouse genome using PCR analyzed microsatellites. Nucleic Acid Res. 8: 41234130.

[23] Hancock JM (1996). Simple sequences and the expanding genome. BioEssays 18: 421-425.

[24] Richard GF, Kerrest A, Dujon B (2008). Comparative genomics and molecular dynamics of DNA repeats in Eukaryotes. Micr. Mol. Bio. Rev. 72 (4): 686-727. doi:10.1128/MMBR.00011-08.

[25] Dib, C., Fauré, S., Fizames, C., Samson, D., Drouot, N., Vignal, A., Millasseau, P., Marc, S., Hazan, J., Seboun, E., Lathrop, M., Gyapay, G., Morissette, J., Weissenbach, J., (1996). A comprehensive genetic map of the human genome based on 5264 microsatellites, Nature (London) 380, 152-154.

[26] Riccio, A., Aaltonen, L., Godwin, A., Loukola, A., Percesepe, A., Salovaara, R., Masciullo, V., Genuardi, M., Paravatou-Petsotas, M., Bassi, D., Ruggeri, B., Andres, J., Klein-Szanto, P., Testa, J., Neri, G., Bellacosa, A. (1999). The DNA repair gene MBD4 (MED1) is mutated in human carcinomas with microsatellite instability, Nature Genet. 23, 266-268.

[27] Sainudiin, R., Durrett, R.T., Aquadro, C.F., Nielsen, R. (2004). Microsatellite mutation models: Insights from a comparison of humans and chimpanzees, Genetics 168, 383-395.

[28] Fu, Y.H., Kuhl, D.A.P., Pizzuti, A., Pieretti, M., Sutcliffe, J., Richards, S., Verkerk, A., Holden, J., Fenwick Jr, R., Warren, S.T., Oostra, B.A., Nelson, D.L., Caskey, C.T. (1991).Variation of the CGG repeat at the fragile $X$ site results in genetic instability: Resolution of the Sherman paradox, Cell 67, 1047-1058.

[29] Cooper, W.G. (1995). Evolutionary origin of expandable G C rich triplet repeat DNA sequences, Biochem. Genet. 33, 173-181. 
W. Grant Cooper; Evolution Via EPR-Entanglement Algorithm. Journal of Biomedical Engineering and Medical Imaging, Volume 4, No 2, April (2017), pp 43-95

[30] Campuzano, V., Montermini, L., Moltò, M., Pianese, L., Cossée, M., Cavalcanti, F., Monros, E., Rodius, F., Duclos, F., Monticelli, A., Zara, F., Cañizares, J., Koutnikova, H., Bidichandani, S., Gellera, C., Brice, A., Trouillas, P., De Michele, G., Filla, A., De Frutos, R., Palau, F., Patel, P., Di Donato, S., Mandel, J.-L., Cocozza, S., Koenig, M., Pandolfo, M. (1996). Friedreich's ataxia: autosomal recessive disease caused by an intronic GAA triplet repeat expansion, Science 271, 1423-1427.

[31] Bidichandani, S.I., Purandare, S.M., Taylor, E.E., Gumin, G., Machkas, H., Harati, Y., Gibbs, R.A., Ashizawa, T., Patel, P.I. (1999). Somatic sequence variation at the Friedreich ataxia locus includes complete contraction of the expanded GAA triplet repeat significant length variation in serially passaged lymphoblasts and enhanced mutagenesis in the flanking sequences, Hum. Mol. Genet. 8, 2425-2436.

[32] Semaka A, Creighton S, Warby S, Hayden MR (2006) Predictive testing for Huntington's disease: interpretation and significance of intermediate alleles. Clin. Genet.70: 283-294.

[34] Sutherland, G.R., Richards, R.I. (1995). Simple tandem DNA repeats and human genetic diseases, Proc. Natl. Acad. Sci. USA 92, 3636- 3641.

[35] Kruglyak, S., Durrett, R.T., Shug, M.D., Aquadro, C.F. (1998). Equilibrium distributions of microsatellite repeat length resulting from a balance between slippage events and point mutations, Proc. Natl. Acad. Sci USA 95, 10774-10778.

[36] La Spada, A.R., Richards, R.I., Wieringa, B. (2004). Dynamic mutations on the move in Banff. Nature Genet. 36, 667-670.

[37] Pearson, C.E., Edamura, K.N., Cleary, J.D. (2005). Repeat instability: Mechanisms of dynamic mutation. Nat. Rev. Genet. 6, 729-742.

[38] Petruska J, Hartenstine MJ, Goodman, MF (1998). Analysis of strand slippage in DNA polymerase expansions of CAG/CTG triplet repeats associated with neurodegenerative disease. J. Biol. Chem. 273: 5204-10.

[39] Santibáñez-Koref, M.F., Gangeswaran, R., Hancock, J.M. (2001). A relationship between lengths of microsatellites and nearby substitution rates in mammalian genomes. Mol. Biol. Evol 18, 2119-2123.

[40] Amos W (2010). Mutation biases and mutation rate variation around very short human microsatellites revealed by human-chimpanzee-orangutan genomic sequence alignments. J. Mol. Evol. 71: 192-201. doi:10.1007/s00239-010-9377-4.

[41] Rubinsztein DC, Amos W, Cooper G (1999). Microsatellite and trinucleotide-repeat evolution: evidence for mutational bias and different rates of evolution in different lineages. Phil. Trans. R. Soc. Lond. B.354, 1095-1099. 
[42] Schlötterer, C. (2000). Evolutionary dynamics of microsatellite DNA. Chromosoma 109, 365-371.

[43] Li YC, Korol AB, Fahima T, Nevo, E (2004). Microsatellites within genes: Structure, function and evolution, Mol. Biol. Evol. 21, 991-1007.

[44] Matsuura, T., Fang, P., Pearson, C.E., Jayakar, P., Ashizawa, T., Roa, B.B. Nelson, D.L. (2006). Interruptions in the expanded ATTCT repeat of spinocerebellar ataxia type 10: Repeat purity as a disease modifier? Am. J. Hum. Genet. 78, 125-129.

[45] Estoup, A., Jarne, P., Cornuet, J.M. (2002). Homoplasy and mutation model at microsatellite loci and their consequences for populating genetic analysis, Mol. Ecol. 11, 1591-1604.

[46] Pearson, CE (2011). Repeat associated non-ATG translation initiation: one DNA, two transcripts, seven reading frames, potentially nine toxic entities! PLoS Genet. 7:e1002018.

[47] Forster P., Hohoff C., Dunkelmann B., Schürenkamp M., Pfeiffer H., Neuhuber F., Brinkmann B. (2015). Elevated germline mutation rate in teenage fathers. Proc. R. Soc. B. 282 (1803): 20142898. doi:10.1098/rspb.2014.2898.

[48] Amos W (2016). Heterozygosity increases microsatellite mutation rate. Biol. Lett. 12: 20150929. doi:10.1098/rsbl.2015.0929.

[49] Bromham, L., Penny, D. (2003). The modern molecular clock. Nat. Rev. Genet. 4, 216-224.

[50] Shepherd, L.D., Lambert, D.M. (2005). Mutational bias in penguin microsatellite DNA. J. Hered. 96, 566-571.

[51] Rubinsztein, D.C., Amos, W., Leggo, J., Goodburn, S., Jain, S., Li, S.-H., Margolis, R., Ross, C., FergusonSmith, M. (1995). Microsatellite evolution - evidence for directionality and variation in rate between species, Nature Genet. 10, 337-343.

[52] Amos, W., Sawcer, S.J., Feakes, R.W., Rubinsztein, D.C. (1996). Microsatellites show mutational bias and heterozygote instability, Nature Genet. 13, 390-391.

[53] Primmer, C.R., Ellegren, H., Saino, N., Møller, A.P. (1996). Directional evolution in germline microsatellite mutations, Nature Genet. 13, 391-393.

[54] Brinkmann B, Klintschar M, Neuhuber F, Huhne J, Rolf B (1998). Mutation Rate in Human Microsatellites: Influence of the Structure and Length of the Tandem Repeat. Am J Hum Genet. 62 (6): 1408-1415. doi:10.1086/301869.

[55] Tautz D, Schlötterer C (1994). Simple sequences. Current Opinion in Genetics \& Development. 4 (6): 832-837. doi:10.1016/0959-437X(94)90067-1. 
W. Grant Cooper; Evolution Via EPR-Entanglement Algorithm. Journal of Biomedical Engineering and Medical Imaging, Volume 4, No 2, April (2017), pp 43-95

[56] Brock, G.J., Anderson, N.H., Moncktron, D.G. (1999). Cis-acting modifiers of expanded CAG/CTG triplet repeat expandability: associations with flanking GC content and proximity to $\mathrm{CpG}$ islands. Hum. Mol. Genet. 8, 1061-1067.

[57] Ohta T, Kimura M (1973). A model of mutation appropriate to estimate the number of electrophoretically detectable alleles in a finite population, Genet. Res. 22, 201-204.

[58] Watson J, Baker T, Bell S, Gann A, Levine M, Losick R. (2013) Molecular Biology of the Gene, 7th ed. Menlo Park, CA: Benjamin-Cummings.

[59] Tegmark M, Wheeler JA (2003) 100 years of the Quantum. Sci. Am. 284:68-75.

[60] Pfaff W, Taminiau TH, Robledo L, Bemien H, Markham M, Twitchen DJ, Hanson R, (2013) Demonstration of entanglement-by-measurement of solid-state qubits. Nature Physics 9: 29-33. doi:10.1038/nphys2444.

[61] de Vicente JI, Spee C, Kraus B (2013) Maximally entangled set of multipartite quantum states. Phys. Rev. Lett. 111: 110502.

[62] Amico L, Fazio R, Osterloh A, Vedral V (2008) Entanglement in many body systems. Rev. Mod. Phys. 80: 517-576.

[63] Xu GF, Zhang J, Tong DM, Sjöqvist E, Kwek LC (2012) Nonadiabatic holonomic quantum computation in decoherence-free subspaces. Phys Rev Lett. 109:170501.

[64] Xu G, Long G (2014) Universal nonadiabatic geometric gates in two-qubit decoherence-free subspaces. Sci Rep. 4:6814.

[65] Suter D, Álvarez GA (2016) Protecting quantum information against environmental noise. Rev. Mod. Phys. 88, 041001.

[66] Goldman N, Tamblyn I (2013) Prebiotic chemistry within a simple impacting icy mixture. J. Phys. Chem. A. 117: 5124.

[67] Koonin EV, Senkevich T, Dolja VV (2006). The ancient virus world and the evolution of cells. Biol. Direct 1: 29.

[68] Shelke SA, Piccirilli JA (2014) Origins of life: RNA made its own mirror image. Nature 515, 347-348. Doi:10.1038/nature13935.

[69] Cech TR (2012) The RNA Worlds in Context. Cold Spring Harb. Perspect. Biol. 4: a006742.

[70] Noller HF (2012) Evolution of protein synthesis from an RNA world. Cold Spring Harb Perspect Biol 4:a003681.

[71] Reif. F (1965) Fundamentals of Statistical and Thermal Physics. McGraw Hill, New York. 
[72] Scheiner S (1997) Hydrogen Bonding. A Theoretical Perspective. Oxford University Press, Oxford.

[73] Wing R, Drew H, Takano T, Broka C, Tanaka S, Itakura K, Dickerson R (1980) Crystal structure analysis of a complete turn of B-DNA. Nature 287:755-756.

[74] Monz T, Kim K, Villar AS, Schindler P, Chwalla M, Riebe M, Roos CF, Häffner H, Hänsel W, Hennrich M, Blatt $R$ (2009) Realization of universal ion-trap quantum computation with decoherence-free qubits. Phys Rev Lett. 103:200503.

[75] Tegmark M (2000) The importance of quantum decoherence in brain processes. Phys. Rev. E 61: 4194-4206.

[76] Zurek WH (2009) Quantum Darwinism, Nat. Phys. 5:181-188.

[77] Takeuchi N, Hogeweg P, Koonin E.V (2011) On the origin of DNA genomes: Evolution of the division of labor between template and catalyst in model replicator systems. PLoS Comput, Biol. 7: Artn. e2002024.

[78] Richards, R (2001) Dynamic mutations: a decade of unstable expanded repeats in human genetic disease, Mol. Hum. Genet.10: 2187-2194.

[79] Miller ES, Kutter E, Mosig G, Arisaka F, Kunisawa T, Rüger W (2003) Bacteriophage T4 genome. Microbiol. Mol. Biol. Rev. 67: 86-156.

[80] Ripley LS (1988) Estimation of in-vivo miscoding rates. Quantitative behavior of two classes of heat-induced DNA lesions. J. Mol. Biol. 202: 17-34.

[81] Baltz RH, Bingham PM, Drake J.W (1976) Heat mutagenesis in bacteriophage T4: The transition pathway. Proc. Natl. Acad. Sci. USA. 73:1269-1273.

[82] Bingham PM, Baltz RH, Ripley LS, Drake JW (1976) Heat mutagenesis in bacteriophage T4: The transversion pathway. Proc. Natl. Acad. Sci. USA, 73: 4159-4163.

[83] Drake JW, McGuire J (1967) Characteristics of mutations appearing spontaneously in extracellular particles of bacteriophage T4. Genetics 55: 387-398.

[84] Arndt M, Juffmann T, Vedral V (2009) Quantum physics meets biology. HFSP J. 3: 386-400.

[85] Wiseman HM, Eisert J (2008) Nontrivial quantum effects in biology: A skeptical physicist's view. In Quantum Aspects of Life, ed. by D Abbott, PCW Davies, AK Pati. Imperial College Press, London, pp. 381-402.

[86] Ball P (2011) Physics of life: The dawn of quantum biology. Nature 474: 272-274.

[87] Vattay G, Kauffman S, Niiranen S (2014) Quantum biology on the edge of quantum chaos. PLoS One 9:89017. 
W. Grant Cooper; Evolution Via EPR-Entanglement Algorithm. Journal of Biomedical Engineering and Medical Imaging, Volume 4, No 2, April (2017), pp 43-95

[88] 88. Lambert N, Chen Y-N, Cheng Y-C, Li C-M, Chen G-Y Nori F (2013) Quantum biology. Nature Phys. 9:10-18. Doi: 10.1038/nphys2474.

[89] Moheni M, Omar Y (2014) eds. Quantum Effects in Biology. Cambridge University Press, Cambridge, UK

[90] Tamulis A, Grigalavicius M (2014) Quantum entanglement in photoactive prebiotic systems. Syst Synth Biol.8:117-140.

[91] Gauger EM, Rieper E, Morton JJL, Benjamin SC, Vedral V (2011) Sustained quantum coherence and entanglement in the avian compass. Phys. Rev. Lett. 106, 040503.

[92] Topal, M.D., Fresco, J.R (1976) Complementary base pairing and the origin of base substitutions. Nature 263: 285-289.

[93] Kouri DJ (2014) Harmonic oscillators, Heisenberg's uncertainty principle and simultaneous measurement precision for position and momentum. arXiv:1409.2468v2 [quant-ph].

[94] Wootters WK, Zurek WH (1982) A single quantum state cannot be cloned. Nature 299: 802-803.

[95] Kricker M, Drake JW (1990) Heat mutagenesis in bacteriophage T4: Another walk down the transversion pathway. J. Bacteriol. 172: 3037-3039.

[96] Drake JW, Ripley LS (1994) Mutagenesis. In JD Karam (Ed.) Molecular Biology of Bacteriophage T4, American Society for Microbiology, Washington DC, pp 98-124.

[97] Drake JW, Charlesworth B, Charlesworth D, Crow JF (1998) Rates of spontaneous mutation. Genetics 148:1667-1686 (see p. 1671).

[98] Benzer S (1961) On the topography of the genetic fine structure. Proc. Natl. Acad. Sci. USA 47:403-415.

[99] Wilde, MM (2013) Quantum Information Theory, Cambridge University Press, Cambridge.

[100] Davies, P.C.W (2004) Does quantum mechanics play a non-trivial role in life? BioSystems 78:6979.

[101] Goel A, Astumian RD, Herschbach D (2003) Tuning and switching a DNA polymerase motor with mechanical tension. Proc. Natl. Acad. Sci. USA 98, 8485-8491.

[102] Wigner, EP (1957) Relativistic invariance and quantum phenomena. Rev. Mod. Phys. 29: 255268.

[103] Koonin EV (2012) The Logic of Chance. The Nature and Origin of Biological Evolution. Pearson, FT Press. Upper Saddle River, New Jersey. 
[104] Nenguke T, Aladjem M, Gusella J, Wexler N, Arnheim N (2003) Candidate DNA replication initiation regions at human trinucleotide repeat disease loci. Hum. Mol. Genet. 12:1021-1028.

[105] Sawaya SM, Lennon D, Buschiazzo E, Gemmell N, Minin V (2012). Measuring Microsatellite Conservation in Mammalian Evolution with a Phylogenetic Birth-Death Model. Genome Biol Evol. 4:748-759.

[106] Varela MA, Amos W (2010) Heterogeneous distribution of SNPs in the human genome: Microsatellites as predictors of nucleotide diversity and divergence. Genomics 95:151-159.

[107] Kumar S, Hedges, SB (1998) A molecular timescale for vertebrate evolution. Nature 392:917-920.

[108] Higham CF, Morales F, Cobbold CA, Haydon D, Monckton DG (2012) High levels of somatic DNA diversity at the myotonic dystrophy type 1 locus are driven by ultra-frequent expansion and contraction mutations. Hum. Mol. Genet. 21, 2450-2463.

[109] Voineagu I, Surka CF, Shishkin AA, Krasilnikova MM, Mirkin, SM (2009) Replisome stalling and stabilization at CGG repeats, which are responsible for chromosomal fragility, Nat Struct Mol Biol 16: 226-228.

[110] Snowdon DA, Kane RL, Beeson WL, Burke GL, Sprafka JM, Potter J, Iso H, Jacobs Jr DR, Phillips RL (1989) Is early natural menopause a biologic marker of health and aging? Am. J. Public Health 79: 709-714.

[111] Ehrenberg L, von Ehrenstein G, Hedgran A (1957) Gonad temperature and spontaneous mutation rate in man. Nature 180, 1433-1434.

[112] Torrellas G, Maciá E (2012) Twist-radial normal mode analysis in double-stranded DNA chains. Phys. Lett. A 376:3407-3410.

[113] Cooper WG (1979) Proton transitions in hydrogen bonds of DNA. A first order perturbation model Int. J. Quantum Chem. Quantum Biol. Symp. 6:171.

[114] Gusella JF, MacDonald ME, Ambrose CM, Duyao MP (1993) Molecular genetics of Huntington's disease. Arch. Neurol. 50:1157-1163.

[115] Semaka A, Creighton S, Warby S, Hayden MR (2006) Predictive testing for Huntington's disease: interpretation and significance of intermediate alleles. Clin. Genet.70: 283-294.

[116] Chung M, Ranum L, Duvick L, Servadio A, Zoghb H, Orr H (1993) Evidence for a mechanism predisposing to intergenerational CAG repeat instability in spinocerebellar ataxia type I. Nat. Genet. 5: 254-258.

[117] Koide R, Ikeuchi T, Onodera O, Tanaka H, Igarashi S, Endo K, Takahashi H, Kondo R, Ishikawa A. Hayashi,T, Saito M. Tomoda A, Miike T, Naito H, Ikuta F, Tsuji S (1994) Unstable expansion of CAG repeats in hereditary dentatorubral-pallidoluysian atrophy. Nat. Genet. 6:9-13. 
W. Grant Cooper; Evolution Via EPR-Entanglement Algorithm. Journal of Biomedical Engineering and Medical Imaging, Volume 4, No 2, April (2017), pp 43-95

[118] Zhang L, Leeflang EP, Yu J, Arnheim N (1994) Studying human mutations by sperm typing: instability of CAG trinucleotide repeats in human androgen receptor gene, Nature Genet. 7, 531-535.

[119] Rosenberg SM (2001) Evolving responsively: adaptive mutations. Nat. Rev. Genet. 2:504-515.

[120] Feng D-F, Cho G, Doolittle RF (1997) Determining divergence times with a protein clock: Update and reevaluation. Proc. Natl. Acad. Sci. 94:13028-13033.

[121] Bozic I, Antal T, Ohtsuki H, Carter H, Kim D, Chen S, Karachin R, Kinzler KW, Vogelstein B, Nowak M.A (2010) Accumulation of driver and passenger mutations during tumor progression. Proc. Natl. Acad. Sci 107: 18545-18550.

[122] Illingworth CJR, Mustonen V (2011) Distinguishing driver and passenger mutations in an evolutionary history categorized by inference. Genetics 189:989-1000.

[123] Mosconi L, Rinne JO, Tsui WH, Berti V, Li Y, Wang H, Murray J, Scheinin N, Nagren K, Williams S, Glodzik L, De Santi S, Vallabhajosula S, de Leon M.J (2010) Increased fibrillar amyloid- $\beta$ burden in normal individuals with a family history of late-onset Alzheimer's. Proc. Natl. Acad. Sci. USA. 107:59495954.

[124] Pifer PM, Yates EA, Legleiter J (2011) Point mutations in $A \beta$ result in the formation of distinct polymorph aggregates in the presence of lipid bilayers, PLoS One 6: e16248.

[125] Cruchaga C, Haller G, Chakraverty S, Mayo K, Vallania FLM, Mitra RD, Faber K, Williamson J, Bird T, Diaz-Arrastia R, Foround TM, Boeve BF, Graff-Radford NR, St. Jean P, Lawson M, Ehm MG, Mayeux,R, Goate AM (2012) Rare variants in APP, PSEN1 and PSEN2 increase risk for AD in late-onset Alzheimer's disease families. PLoS One 7: e31039.

[126] Lujambio A, Portela A, Liz J, Melo SA, Rossi S, Spizzo R, Croce CM, Calin GA, Esteller M (2010) CpG island hypermethylation-associated silencing of non-coding RNAs transcribed from ultraconserved regions in human cancer. Oncogene 29:6390-6401.

[127] Huang Y, Shen X.J, Zou Q, Wang SP, Tang SM, Zhang G.Z (2011) Biological functions of microRNAs: a review. J. Physiol. Biochem 67: 129-139.

[128] Kumar. S (2005) Molecular clocks: four decades of evolution. Nat. Rev. Genet. 6: 654-662. 